Supporting Information for:

\title{
Mechanistic Insights into C-H Borylation of Arenes with Organoiridium Catalysts Embedded in a Microporous Metal- Organic Framework
}

Zoha H. Syed, ${ }^{a, b}$ Zhihengyu Chen, ${ }^{c}$ Karam B. Idrees, ${ }^{a}$ Timothy A. Goetjen, ${ }^{a}$ Evan C. Wegener, ${ }^{b}$ Xuan Zhang, ${ }^{a}$ Karena W. Chapman, ${ }^{c}$ David M. Kaphan, ${ }^{\mathrm{b}, *}$ Massimiliano Delferro, ${ }^{\mathrm{b}, *}$ Omar K. Farha ${ }^{\mathrm{a}, *}$

*o-farha@u.northwestern.edu *delferro@anl.gov *kaphand@anl.gov

a Department of Chemistry and International Institute of Nanotechnology, Northwestern University, 2145 Sheridan Road, Evanston, Illinois 60208, United States

${ }^{b}$ Chemical Sciences and Engineering Division, Argonne National Laboratory, Lemont, Illinois 60439, United States

c Department of Chemistry, Stony Brook University, Stony Brook, New York 11764, United States 


\section{Table of Contents}

$\begin{array}{ll}\text { General methods and materials } & \text { S9 }\end{array}$

$\begin{array}{ll}\text { Instrumentation and sample preparation } & \text { S9 }\end{array}$

- Solution-phase nuclear magnetic resonance (NMR) spectra $\left({ }^{1} \mathrm{H}\right) \quad$ S9

- Diffuse Reflectance Infrared Fourier Transform Spectroscopy (DRIFTS) s9 spectra

- Scanning electron microscopy (SEM) $\quad \mathbf{S 1 0}$

- X-ray photoelectron spectroscopy $\quad$ S10

- Inductively coupled plasma-optical emission spectroscopy (ICP-OES) S10

- Diffraction patterns for Pair Distribution Functions (PDF) and Differential Envelope Densities (DED)

- Gas chromatography-flame ionization detector (GC-FID) $\mathbf{S 1 1}$

- Benzene vapor-phase single-component adsorption measurements $\quad \mathbf{S 1 1}$

- X-ray Absorption Spectroscopy (XAS) measurements $\quad$ S11

$\begin{array}{ll}\text { Synthesis of Materials } & \mathbf{S 1 2}\end{array}$

$\begin{array}{ll}\text { - } \operatorname{UiO}-67-\operatorname{mix}(1) & \mathbf{S 1 2}\end{array}$

- UiO-67-mix-Ir(I) (2) $\quad$ S12

- UiO-67-mix-Ir(III) (3) $\quad$ S12

○ Figure S1. Color changes observed in metalation to synthesize $3 \quad \mathbf{S 1 2}$

- UiO-67-mix-Ir post-borylation (4) S13

- UiO-67-mix-Ir post-borylation (4) for PDF/DED analysis $\quad \mathbf{S 1 3}$

- UiO-67-mix-Ir post-borylation (4) for XAS S13

- $\quad($ Phen $) \operatorname{Ir}(\mathrm{COD})(\mathrm{Cl}) \quad$ S13

$\begin{array}{lr}\text { Characterization of Materials } & \mathbf{S 1 4}\end{array}$

- ${ }^{1}$ H NMR Spectra $\quad \mathbf{S 1 4}$ Figure S2. ${ }^{1} \mathrm{H} \quad \mathrm{NMR}$ spectrum of (Phen) $\operatorname{Ir}(\mathrm{COD})(\mathrm{Cl})$ in $\mathrm{CDCl}_{3} \quad \mathbf{S 1 4}$
synthesized according to previously published procedures. 
- Powder XRD Patterns

$\circ \quad$ Figure S3. Powder XRD patterns of 1, 2, 3, 4 showing that crystallinity and phase purity is maintained after treatments.

- $\mathrm{N}_{2}$ Sorption Data

$\circ$ Figure S4. $\mathrm{N}_{2}$ adsorption/desorption isotherm of UiO-67-mix (1) collected at $77 \mathrm{~K}$

- Figure S5. DFT Pore Size Distribution of UiO-67-mix (1) collected at 77 K

S16

$\circ$ Figure S6. $\mathrm{N}_{2}$ adsorption/desorption isotherm of UiO-67-mix-Ir(I) (2) collected at $77 \mathrm{~K}$

Figure S7. DFT Pore Size Distribution of UiO-67-mix-Ir(I) (2) collected at $77 \mathrm{~K}$

517

S17

- Figure S8. $\mathrm{N}_{2}$ adsorption/desorption isotherm of UiO-67-mix-Ir(III) (3) collected at $77 \mathrm{~K}$

$\mathrm{S} 18$

- Figure S9. DFT Pore Size Distribution of UiO-67-mix-Ir(III) (3) collected at $77 \mathrm{~K}$

S18

- Figure S10. $\mathrm{N}_{2}$ adsorption/desorption isotherm of UiO-67-mix-Ir postborylation (4) collected at $77 \mathrm{~K}$

S19

- Figure S11. DFT Pore Size Distribution of UiO-67-mix-Ir post-borylation (4) collected at $77 \mathrm{~K}$

S19

- SEM Images

$\mathrm{S} 20$

- Figure S12. SEM image of UiO-67-mix-Ir(III) (3)

S20

○ Figure S13. SEM image of UiO-67-mix-Ir post-borylation (4)

S20

- DRIFTS Spectra

S21

$\circ \quad$ Figure S14. DRIFTS of UiO-67-mix (1)

S21

○ Figure S15. DRIFTS of UiO-67-mix-Ir(I) (2)

S22

○ Figure S16. DRIFTS of UiO-67-mix-Ir(III) (3)

S23

○ Figure S17. DRIFTS of UiO-67-mix-Ir post-borylation (4)

S24

- ${ }^{1} \mathrm{H}$ NMR Digestion

S25

- Figure S18. ${ }^{1} \mathrm{H}$ NMR acid digestion of UiO-67-mix $\left(\mathrm{D}_{2} \mathrm{SO}_{4} / \mathrm{DMSO}-d_{6}\right)$

S25

- ICP Data

S25

○ Table S1. Average concentrations of acid-digested ICP sample of $\mathbf{3} \quad \mathbf{S 2 5}$

- Table S2. Calculated analyte concentrations and weight percent based on Table S1

S25

- Table S3. Average concentrations of acid-digested (quartz vessel) ICP sample of $\mathbf{3}$ 
- Table S4. Calculated analyte concentrations based on Table S3

○ Table S5. Average concentrations of acid-digested ICP sample of $4 \quad \mathbf{S 2 6}$

- Table S6. Calculated analyte concentrations and weight percent based on Table S5

○ Table S7. Calculated analyte concentrations based on Table S5

- XAS Data

- Figure S19. Ir L3 edge XANES of UiO-67-mix-Ir(III), 3, $[\operatorname{Ir}(\mathrm{COD}) \mathrm{Cl}]_{2}$, and (Phen) $\operatorname{Ir}(\mathrm{COD})(\mathrm{Cl})$

- Figure S20. Ir L3 edge XANES of UiO-67-mix-Ir(III), 3, and UiO-67-mixIr post-borylation, 4

$\mathbf{S 2 7}$

Figure S21. The magnitude and imaginary part of the $\mathrm{k}^{2}$-weighted EXAFS of UiO-67-mix-Ir(III), 3

$\circ \quad$ Figure S22. The magnitude part of the $\mathrm{k}^{2}$-weighted EXAFS of UiO-67mix-Ir(III), 3 and $[\operatorname{Ir}(\mathrm{COD}) \mathrm{Cl}]_{2}$

- Figure S23. The magnitude and imaginary part of the $k^{2}$-weighted EXAFS of UiO-67-mix-Ir post-borylation, 4.

$\circ \quad$ Figure S24. The magnitude part of the $\mathrm{k}^{2}$-weighted EXAFS o UiO-67mix-Ir(III), 3 and (Phen) $\operatorname{Ir}(\mathrm{COD})(\mathrm{Cl})$

○ Table S8. Ir LIII Edge EXAFS Fitting Parameters

S27

S28

S28

S29

S29

S30

- XPS Data

S30

- Figure S25. XPS spectra showing the binding energy of UiO-67-mix$\operatorname{Ir}(\mathrm{I})$ at the $\mathrm{Zr} 3 \mathrm{~d}$ region

S30

- Figure S26. XPS spectra showing the binding energy of UiO-67-mix$\operatorname{Ir}(\mathrm{I})$ at the Ir $4 \mathrm{f}$ region

- Figure S27. XPS spectra showing the binding energy of UiO-67-mix$\operatorname{Ir}(\mathrm{I})$ at the $\mathrm{Cl} 2 \mathrm{p}$ region

$\circ$ Figure S28. XPS spectra showing the binding energy of UiO-67-mix$\operatorname{Ir}(\mathrm{I})$ at the $O 1 \mathrm{~s}$ region

$\circ$ Figure S29. XPS spectra showing the binding energy of UiO-67-mix$\operatorname{Ir}(I)$ at the $C 1$ s region

- Figure S30. XPS spectra showing the binding energy of UiO-67-mix$\operatorname{Ir}(\mathrm{III})$ at the $\mathrm{Zr} 3 \mathrm{~d}$ region

$\circ$ Figure S31. XPS spectra showing the binding energy of UiO-67-mixIr(III) at the Ir $4 \mathrm{f}$ region

- Figure S32. XPS spectra showing the binding energy of UiO-67-mix$\mathrm{Ir}(\mathrm{III})$ at the $\mathrm{Cl} 2 \mathrm{p}$ region

$\circ$ Figure S33. XPS spectra showing the binding energy of UiO-67-mix$\operatorname{Ir}(\mathrm{III})$ at the $\mathrm{O}$ 1s region

- Figure S34. XPS spectra showing the binding energy of UiO-67-mix-Ir post-borylation at the $\mathrm{Zr} 3 \mathrm{~d}$ region

$\circ \quad$ Figure S35. XPS spectra showing the binding energy of UiO-67-mix-Ir post-borylation at the Ir $4 \mathrm{f}$ region

S31

S31

S32

S32

S33

S33

S34

S34

S35

S35

- Figure S36. XPS spectra showing the binding energy of UiO-67-mix-Ir post-borylation at the $\mathrm{Cl} 2 \mathrm{p}$ region 
- Figure S37. XPS spectra showing the binding energy of UiO-67-mix-Ir post-borylation at the $O 1$ s region

- Figure S38. XPS spectra showing the binding energy of UiO-67-mix$\operatorname{Ir}(\mathrm{III})$-post-boryl at the $\mathrm{C}$ 1s region

- PDF Data

- Figure S39. PDF analysis of x-ray diffraction patterns for UiO-67-mix and Ir-metalated samples

- dPDF Data

○ Table S9. dPDF data for UiO-67-mix-Ir(I), $2 \quad$ S38

○ Table S10. dPDF data for UiO-67-mix-Ir(III), $3 \quad$ S38

○ Table S11. dPDF data for UiO-67-mix-Ir post-borylation, $4 \quad$ S38

- DED Data

- Figure S40. DED analysis of UiO-67-mix-Ir(I), full view, solid bulbs represent electron density

○ Figure S41. DED analysis of UiO-67-mix-Ir(I), zoomed-in ligand view, solid bulbs represent electron density

S39

- Figure S42. DED analysis of UiO-67-mix-Ir(III), full view, solid bulbs represent electron density

$\circ$ Figure S43. DED analysis of UiO-67-mix-Ir(III), zoomed-in ligand view, solid bulbs represent electron density

- Figure S44. DED analysis of UiO-67-mix-Ir post-borylation, full view, solid bulbs represent electron density

- Figure S45. DED analysis of UiO-67-mix-Ir post-borylation, zoomed-in ligand view, solid bulbs represent electron density

- Benzene Sorption Data

S41

S41

$\circ \quad$ Figure S46. Vapor-phase benzene sorption ( $1^{\text {st }}$ and $2^{\text {nd }}$ cycle) with UiO67-mix

- Figure S47. Vapor-phase benzene sorption ( $1^{\text {st }}$ and $2^{\text {nd }}$ cycle) with UiO67-mix-Ir(III)

- Figure S48. Borylation of benzene forming an equivalent of PhBPin and HBPin, the in situ-generated HBPin can then be used for borylation of another equivalent of benzene to form $\mathrm{PhBPin}$ and $\mathrm{H}_{2}$

- General procedure for kinetic monitoring of benzene borylation reaction with $\mathrm{B}_{2} \mathrm{Pin}_{2}$ and $\mathrm{HBPin}$

$\bigcirc$ Table S12. Conditions for Kinetic Monitoring of Benzene Borylation with HBPin

$\circ$ Figure S49. Effect of added [HBPin] on consumption of $\left[\mathrm{B}_{2} \mathrm{Pin}_{2}\right]$ and formation of [PhBPin] overlaid with kinetic plots from Table S19 entries 1-3.

- General procedure for heterogeneous benzene borylation reaction with HBPin as the boron source

\section{S44}


- Table S13. Formation of PhBPin with HBPin as the Boron Source

- General procedure for kinetic monitoring of benzene borylation reaction with $2 \mathbf{S 4 4}$

- Table S14. Conditions for Kinetic Monitoring of Benzene Borylation with 2

○ Figure S50. Benzene borylation with 2

- General procedure for hot filtration test of heterogeneous benzene borylation reaction

○ Table S15. Product Distribution of Hot Filtration Test

S45

S46

- General procedure for native MOF control reaction of heterogeneous benzene borylation reaction

\section{S46}

- Table S16. Product Distribution of Native MOF Control Reaction

S46

- General procedure for no catalyst control reaction of benzene borylation reactions

- Table S17. Product Distribution of No Catalyst Control Reaction

\section{S46}

S46

- General procedure for kinetic monitoring of heterogeneous benzene borylation reactions

- Table S18. Conditions for Kinetic Monitoring of Benzene Borylation

S47

- Equation 1. Calculation of mass balance as a percentage based on limiting reagent $\mathrm{B}_{2} \mathrm{Pin}_{2}$ for benzene borylation kinetic plots

S47

Figure S51. A comparison of benzene borylation with two batches of catalyst

\section{S47}

S48

○ Figure S52. Kinetic plots for Table S18, Entry 1

S48

○ Figure S53. Kinetic plots for Table S18, Entry 2

S49

○ Figure S54. Kinetic plots for Table S18, Entry 3

S49

○ Figure S55. Kinetic plots for Table S18, Entry 4

S49

○ Figure S56. Kinetic plots for Table S18, Entry 5

S50

○ Figure S57. Kinetic plots for Table S18, Entry 6

○ Figure S58. Kinetic plots for Table S18, Entry 7

○ Figure S59. Kinetic plots for Table S18, Entry 8

○ Figure S60. Kinetic plots for Table S18, Entry 9

- Equation 2. Mathematical expression to obtain each value of the normalized time scale for VTNA to deduce rate dependence on $\left[\mathrm{B}_{2} \mathrm{Pin}_{2}\right]$ in the borylation reaction

- Figure S61. VTNA plots for determination of rate dependence on $\mathrm{B}_{2} \mathrm{Pin}_{2}$ (Table S18. Entries 2, 5, 6, 7), -0.5 represents the plot fit to -0.5 order

\section{S51}

S52 
- Figure S62. VTNA plots for determination of rate dependence on $\mathrm{B}_{2} \mathrm{Pin}_{2}$ (Table S18. Entries 2, 5, 6, 7), - 0.1 represents the plot fit to -0.1 order

- Figure S63. VTNA plots for determination of rate dependence on $\mathrm{B}_{2} \mathrm{Pin}_{2}$ (Table S18. Entries 2, 5, 6, 7), 0 represents the plot fit to 0 order

$\circ$ Figure S64. VTNA plots for determination of rate dependence on $\mathrm{B}_{2} \mathrm{Pin}_{2}$ (Table S18. Entries 2, 5, 6, 7), 1 represents the plot fit to 1 order

$\circ \quad$ Figure S65. VTNA plots for determination of rate dependence on $\mathrm{B}_{2} \mathrm{Pin}_{2}$ (Table S18. Entries 2, 5, 6, 7), 2 represents the plot fit to 2 order

- Equation 3. Mathematical expression to obtain each value of the normalized time scale for VTNA to deduce rate dependence on [Benzene] in the borylation reaction

- Figure S66. VTNA plots for determination of rate dependence on Benzene (Table S18. Entries 1, 2, 3, 4), -0.5 represents the plot fit to 0.5 order

- Figure S67. VTNA plots for determination of rate dependence on Benzene (Table S18. Entries 1, 2, 3, 4), 0.5 represents the plot fit to 0.5 order

- Figure S68. VTNA plots for determination of rate dependence on Benzene (Table S18. Entries 1, 2, 3, 4), 0.75 represents the plot fit to 0.75 order

- Figure S69. VTNA plots for determination of rate dependence on Benzene (Table S18. Entries 1, 2, 3, 4), 1 represents the plot fit to 1 order

- Figure S70. VTNA plots for determination of rate dependence on Benzene (Table S18. Entries 1, 2, 3, 4), 2 represents the plot fit to 2 order

- Equation 4. Mathematical expression to obtain each value of the normalized time scale for VTNA to deduce rate dependence on [Catalyst] in the borylation reaction

- Figure S71. VTNA plots for determination of rate dependence on Catalyst (Table S14. Entries 4, 8, 9), 0 represents the plot fit to 0 order

- Figure S72. VTNA plots for determination of rate dependence on Catalyst (Table S14. Entries 4, 8, 9), 1 represents the plot fit to 1 order

\section{S57}

S58

S58

- Figure S73. Calibration curve for quantification of $\left[\mathrm{B}_{2} \mathrm{Pin}_{2}\right]$ by $\mathrm{GC}-\mathrm{FID}$

Figure S74. Calibration curve for quantification of [PhBPin] by GC-FID

- Figure S75. Calibration curve for quantification of [4-methylboronic acid pinacol ester] by GC-FID

$\circ \quad$ Figure S76. Calibration curve for quantification of [3-methylboronic acid pinacol ester] by GC-FID

- Figure S77. Calibration curve for quantification of [2-methylboronic acid pinacol ester] by GC-FID

- General procedure for kinetic isotope effect of heterogeneous benzene borylation reactions

- Table S19. Conditions for Kinetic Isotope Effect Study of Benzene Borylation

- Table S20. Results for Kinetic Isotope Effect Study of Benzene Borylation (Table S19 Entries 1-3)

- Table S21. Results of Kinetic Isotope Effect Study of Benzene Borylation (Table S19 Entries 4-6) 
- Figure S78. $\mathrm{B}_{2} \mathrm{Pin}_{2}$ consumption in benzene and benzene- $d_{6}$ borylation with error bars reflecting standard deviation (sample) of triplicate runs

- Figure S79. $\mathrm{B}_{2} \mathrm{Pin}_{2}$ consumption and PhBPin formation in benzene, triplicate runs overlaid.

$\circ$ Figure S80. $\mathrm{B}_{2} \mathrm{Pin}_{2}$ consumption and PhBPin formation in benzene- $d_{6}$, triplicate runs overlaid.

- General procedure for heterogeneous toluene borylation reaction

- General procedure for heterogeneous benzene borylation reaction

- General procedure for heterogeneous benzene and toluene competition borylation reaction

S66

- General procedure for homogeneous benzene and toluene competition borylation reaction

S66

- General procedure for size exclusion experiment with $t$-butylbenzene $\quad \mathbf{S 6 6}$

- General procedure for size exclusion experiment with benzene comparison $\quad$ S66

○ Table S22. Size Exclusion Experiment $\quad \mathbf{S 6 7}$

- General procedure for heterogeneous benzene borylation recycling experiment S67

- Figure S81. Comparison of kinetic profiles with fresh and recycled $3 . \quad$ S68 


\section{General Methods and Materials}

Air- and moisture-sensitive procedures were conducted using an MBraun or Vac glovebox under inert atmosphere. Tetrahydrofuran (THF), benzene, and cyclohexane were purchased as Sure/Seal ${ }^{\mathrm{TM}}$ bottles from Sigma-Aldrich and used/stored inside a glovebox under inert atmosphere. Solvents for air- and moisture-sensitive procedures were dried over vacuum/thermally-activated alumina or vacuum/thermally-activated $3 \AA$ molecular sieves. Dimethylsulfoxide (DMSO)- $d_{6}$ and $\mathrm{D}_{2} \mathrm{SO}_{4}$ were purchased from Sigma-Aldrich and used as received. $\mathrm{CDCl}_{3}$ was purchased from Sigma-Aldrich and used after drying over vacuum/thermallyactivated alumina. Bis(1,5-cyclooctadiene)diiridium(I) dichloride $\left([\mathrm{Ir}(\mathrm{COD}) \mathrm{Cl}]_{2}\right)$, bispinacolatodiboron $\left(\mathrm{B}_{2} \mathrm{Pin}_{2}\right)$, and palladium $(\mathrm{II})$ acetate $\left(\mathrm{Pd}(\mathrm{OAc})_{2}\right)$ were purchased from Strem Chemicals Inc and used/stored in a glovebox under inert atmosphere. For the PhenDC linker synthesis, 3,8-dibromo-1,10-phenanthroline was purchased from Ark Pharm, N,Ndimethylformamide (DMF), anhydrous DriSolv ${ }^{\mathrm{TM}}$ was purchased from Fisher Scientific, Xantphos was purchased from Sigma-Aldrich, potassium fluoride (KF) was purchased from Alfa Aesar, and $\mathrm{N}$-formylsaccharin was purchased from TCl America-all were stored and used inside a glovebox under inert atmosphere. Sodium hydroxide $(\mathrm{NaOH})$, hydrochloric acid $(\mathrm{HCl})$, and triethylamine were purchased from Fisher Scientific and used as received. For the UiO-67-mix synthesis, zirconyl chloride octahydrate $\left(\mathrm{ZrOCl}_{2} \cdot 8 \mathrm{H}_{2} \mathrm{O}\right)$, benzoic acid, and biphenyl-4,4'-dicarboxylic acid (BPDC) were purchased from Sigma-Aldrich and used as received. For XAS samples, to prevent interference with hafnium impurity, ultra-pure zirconium dichloride oxide hydrate, Puratronic ${ }^{\mathrm{TM}}$, 99.9985\% (Metal Basis) was purchased from Alfa-Aesar and used as received. Pinacolborane (HBPin) was obtained as a Sure/Seal ${ }^{\mathrm{TM}}$ bottle from Sigma-Aldrich and sparged with $\mathrm{N}_{2}$ prior to transferring to a glovebox under inert atmosphere - it was used without further manipulation.

PhenDC linker and UiO-67-mix were prepared according to our previously reported procedures. ${ }^{1}$ UiO-67-mix-Ir samples were prepared using a modified procedure from what has previously been reported, vide infra. (Phen) $\operatorname{Ir}(\mathrm{COD})(\mathrm{Cl})$ was synthesized according to a previously reported procedure. $^{2}$

To prevent variation in initial rates of the reaction due to changes in catalyst attributes such as particle size, pore architecture, defects etc., data was only compared within a dataset using the same batch of catalyst (see below for further discussion of batch to batch catalyst variation on page S48).

\section{Instrumentation and Sample Preparation}

Solution-phase nuclear magnetic resonance (NMR) spectra $\left({ }^{1} \mathrm{H}\right)$ were collected using a 400 $\mathrm{MHz}$ Bruker Avance III HD Nanobay system equipped with SampleXpress autosampler, Bruker Avance III $600 \mathrm{MHz}$ system, or Bruker Avance III $500 \mathrm{MHz}$ system equipped with SampleXpress autosampler. All spectra were referenced to residual solvent signals. NMR spectra were processed and integrated using Mnova by Mestrelab Research, following phase correction and baseline correction (Whittaker smoother).

Diffuse Reflectance Infrared Fourier Transform Spectroscopy (DRIFTS) spectra were collected using a Thermo Scientific Nicolet iS50 FT-IR spectrometer with an MCT-A detector cooled to $77 \mathrm{~K}$ and a Harrick Scientific Praying Mantis ${ }^{\mathrm{TM}}$ Diffuse Reflectance accessory was used to acquire spectra under air- and moisture-free conditions. DRIFTS samples were prepared inside of a glovebox under inert atmosphere and measured under this atmosphere with $\mathrm{KBr}$ as a 
background. Spectra were subsequently processed in the OMNIC TM series software by Thermo Fisher Scientific using the Kubelka-Munk function.

$\mathbf{N}_{2}$ sorption and Brunauer-Emmett-Teller (BET) Analysis was conducted after activating MOF materials by exchanging into acetone overnight and then activating under high vacuum using a Micromeritics Smart Vac Prep between $25^{\circ} \mathrm{C}$ and $150^{\circ} \mathrm{C}$ between 12 and 24 hours and/or until the low outgas rate is achieved. $\mathrm{N}_{2}$ adsorption/desorption isotherm data were collected on a Micromeritics Tristar II 3020 instrument at $77 \mathrm{~K}$. Pore size distributions were calculated using density functional theory (DFT) (carbon slit-pore model with an $\mathrm{N}_{2}$ kernal).

Scanning electron microscopy (SEM) images were collected on a Hitachi S4800-II cFEG SEM microscope at Northwestern University's EPIC/NUANCE facility. Prior to SEM image collection, samples were coated with $\mathrm{OsO}_{4}$ to $\sim 9 \mathrm{~nm}$ thickness to improve conductivity using a Denton Desk III TSC Sputter Coater.

X-ray photoelectron spectroscopy (XPS) data was collected on a Thermo Scientific ESCALAB $250 \mathrm{Xi}$ (Al Ka radiation, hv = S5 $1486.6 \mathrm{eV}$ ) equipped with an electron flood gun at the KECKII/NUANCE facility at Northwestern University. It was subsequently processed using the Thermo Scientific Avantage Data System software and all spectra were referenced to the C1s peak (284. $8 \mathrm{eV})$.

Inductively coupled plasma-optical emission spectroscopy (ICP-OES) was performed on a computer-controlled (QTEGRA software) Thermo iCap7600 ICP-OES by Thermo Fisher Scientific operating in radial view and equipped with a CETAC 520 autosampler. Each sample was acquired using 5 second visible exposure time and 15 second UV exposure time, running 3 replicates. Samples were then analyzed for Ir (224.268, 212.681, 205.222, and 263.971 nm), Zr (343.823, $327.305,339.198$, and $349.621 \mathrm{~nm})$, and/or B (249.773, 249.678, and $208.959 \mathrm{~nm})$

Quantification of samples with Ir/Zr/B was accomplished via ICP-OES of acid digested samples in quartz vessels. Specifically, the sample was digested in $2 \mathrm{~mL}$ concentrated trace metals grade nitric acid via microwave digestion in a Milestone Ethos EZ Microwave Digestion system at $1200 \mathrm{~W}$. The temperature was ramped to $150{ }^{\circ} \mathrm{C}$ for 10 minutes, held at $150{ }^{\circ} \mathrm{C}$ for 20 minutes, and exhausted for 30 minutes. $0.3 \mathrm{~mL}$ were then pipetted into a VWR® metal-free tube and diluted to $10 \mathrm{~mL}$ with Millipore water, producing a solution that is $3 \%$ nitric acid by volume. Quantitative B standards (40 mL each) were made using $1000 \mathrm{mg} / \mathrm{L}( \pm 3 \mathrm{mg} / \mathrm{L})$ boron standard from Flukaß Analytical and Millipore water in VWR® metal-free tubes (2 ppm, 5 ppm, 10 ppm, 20 ppm). Quantitative Ir standards (40 mL each) were made using $1000 \mu \mathrm{g} / \mathrm{mL}$ iridium standard from Alfa Aesar ${ }^{\circledR}$, Specpure ${ }^{\circledR}$ and Millipore water in VWR® metal-free tubes (2 ppm, 5 ppm, 10 ppm, 20 ppm). Quantitative $\mathrm{Zr}$ standards (40 mL each) were made using $998 \mu \mathrm{g} / \mathrm{mL}( \pm 5 \mu \mathrm{g} / \mathrm{mL}$ ) zirconium standard from Inorganic ${ }^{\mathrm{TM}}$ Ventures and Millipore water in VWR® metal-free tubes (2 ppm, 5 ppm, 10 ppm, 20 ppm). Note, trace boron contamination can come from factors such as residual boron concentrations in Millipore water or handling of samples prior to digestion preparation with tools containing boron such as borosilicate glass.

For samples with just $\mathrm{Ir} / \mathrm{Zr}$, quantification of samples was accomplished via ICP-OES of acid digested samples in $2-5 \mathrm{~mL}$ microwave reaction vials from Biotage ${ }^{\circledR}$. Specifically, the sample was digested in $2 \mathrm{~mL}$ concentrated trace metals grade nitric acid via microwave digestion in a Biotage ${ }^{\circledR}$ SPX microwave reactor at $150{ }^{\circ} \mathrm{C}$ for $15 \mathrm{~min} .0 .3 \mathrm{~mL}$ were then pipetted into a VWR® metal-free tube and diluted to $10 \mathrm{~mL}$ with Millipore water, producing a solution that is $3 \%$ nitric acid by volume. Quantitative Ir standards (40 mL each) were made using $1000 \mu \mathrm{g} / \mathrm{mL}$ iridium 
standard from Alfa Aesar $\AA$, Specpure $\AA$ and Millipore water in VWR $\circledast$ metal-free tubes (2 ppm, 5 ppm, 10 ppm, 20 ppm). Quantitative Zr standards (40 mL each) were made using $998 \mu \mathrm{g} / \mathrm{mL}( \pm 5$ $\mu \mathrm{g} / \mathrm{mL}$ ) zirconium standard from Inorganic ${ }^{\mathrm{TM}}$ Ventures and Millipore water in VWR® metal-free tubes (2 ppm, 5 ppm, 10 ppm, 20 ppm).

Diffraction patterns for Pair Distribution Functions (PDF) and Differential Envelope Densities (DED) were obtained from beamline 11-ID-B at Advanced Photon Source, Argonne National Laboratory with $58.7 \mathrm{keV}(0.2113 \AA) \mathrm{X}$-ray and amorphous silicon-based detector. ${ }^{3}$ Twodimensional images were integrated to one-dimensional data by GSAS-II. ${ }^{4}$ PDFs were obtained from PDFgetX2 ${ }^{5}$ and $x P D F s u i t e^{6}$ with $Q_{\max }=22.5 \AA^{-1}$. Peak information in PDFs were quantified by Fityk ${ }^{7}$ with Gaussian functions. TOPAS ${ }^{8}$ was used for Pawley refinement and extracting diffraction peak intensity. By subtracting PDF of pristine MOFs from the reacted MOFs, differential PDF (dPDF) can give structural information of the guest molecule and how it interacted with the frameworks. DED was generated by calculating and subtracting the structure envelopes in the unit cell, and finally visualized by Chimera. ${ }^{9}$

Gas chromatography-flame ionization detector (GC-FID) was used to analyze reactions. GCFID measurements were conducted using an Agilent Technologies 7820A GC equipped with FID containing an Agilent J\&W HP-5 capillary column (length $=30 \mathrm{~m}$, diameter $=320 \mu \mathrm{m}$, film thickness $=0.25 \mu \mathrm{m}$, Agilent 19091 J-413, equilibration time $=3 \mathrm{~min}$, max temperature $=325^{\circ} \mathrm{C}$ ). Prior to GC analysis, an aliquot of the reaction was diluted with anhydrous cyclohexane in a glovebox under inert atmosphere and solids were separated via filtration using GE Healthcare Whatman $^{\text {TM }}$ Mini-UniPrep ${ }^{\mathrm{TM}}$ Syringeless Filter vials (membrane $=$ Polytetrafluoroethylene (PTFE), septa $=$ polytetrafluoroethylene $($ PTFE) coated silicone rubber, pore size $=0.2 \mu \mathrm{m}$, volume capacity $=0.4 \mathrm{~mL}$, polypropylene housing). The $\mathrm{GC}$ method was as follows: $40^{\circ} \mathrm{C}$ for $1 \mathrm{~min}$, then $10^{\circ} \mathrm{C} / \mathrm{min}$ to $150^{\circ} \mathrm{C}$ for $0 \mathrm{~min}$, then $20^{\circ} \mathrm{C} / \mathrm{min}$ to $300^{\circ} \mathrm{C}$ for $2 \mathrm{~min}$, total run time $=21.5 \mathrm{~min}, 1 \mu \mathrm{L}$ injection volume. Hexadecane was used as an internal standard and all quantification of reactions was conducted using the ratio of analyte peak area / internal standard peak area. This was converted to a ratio of concentrations analyte concentration / internal standard concentration using the $y=m x+b$ equation of a trend line fit to a calibration curve generated from authentic samples of analytes at various concentrations. See below for calibration curves generated for quantification of all catalytic reactions.

Benzene vapor-phase single-component adsorption measurements were collected on a Micromeritics 3Flex instrument for benzene vapor uptake at $65^{\circ} \mathrm{C}$. The samples were thermally activated at $65^{\circ} \mathrm{C}$ under high vacuum on a Smart Vac Prep instrument for the first measurement. To prepare the vaporizer, anhydrous benzene, dried over $3 \AA$ molecular sieves and stored in a glovebox under inert atmosphere, was added to the vaporizer chamber and three freeze-pumpthaw cycles were performed to remove atmospheric impurities from the solvent. The vaporizer was heated to $40^{\circ} \mathrm{C}$ throughout the entire measurement and adsorption points were collected from 0 to $60 \mathrm{mmHg}$ with 20 seconds equilibration interval. In addition, an Iso Controller was used to maintain a constant temperature of $65^{\circ} \mathrm{C}$ for all samples throughout the entire experiment. The desorption isotherm was also collected according to the same parameters above. Following the first measurement, samples were reactivated in-operando at $65^{\circ} \mathrm{C}$ for 3 hours and a second measurement was performed using the same parameters above.

X-ray Absorption Spectroscopy (XAS) measurements were acquired using the Ir L3 edge and conducted on the bending magnet beamline (10BM) of the Materials Research Collaborative Access Team (MRCAT) at the Advanced Photon Source (APS), Argonne National Laboratory. 
Data was collected in transmission mode and samples were ground then pressed into a cylindrical sample holder containing six wells, which formed a self-supported wafer, using boron nitride as needed. The raw spectra were processed with Athena, part of the Demeter software suite,${ }^{10}$ using standard normalization and background subtraction techniques.

\section{Synthesis of Materials}

\section{UiO-67-mix (1)}

UiO-67-mix was prepared according to previously reported procedures. ${ }^{1}$

\section{UiO-67-mix-Ir(I) (2)}

In a glovebox under inert atmosphere, to an oven-dried dram vial was added UiO-67-mix (100 $\mathrm{mg}, \sim 33 \%$ PhenDC incorporation) and $[\operatorname{Ir}(\mathrm{COD}) \mathrm{Cl}]_{2}(30.94 \mathrm{mg}, 0.046 \mathrm{mmol})$. To this was added anhydrous THF $(0.92 \mathrm{~mL})$ to create a suspension that was green. The vial was capped and shaken overnight to facilitate mass transport. The suspension changes from green to blue overnight. Next, the material is soaked in anhydrous THF. The solids are allowed to settle, and the supernatant is decanted. The material is then soaked and washed with pentane and the supernatant is decanted. The material is dried under vacuum at room temperature. It is then evacuated further under high vacuum at $25^{\circ} \mathrm{C}$ on a Smart Vac Prep. It is then transferred without exposure to ambient atmosphere and stored in a glovebox under inert atmosphere.

\section{UiO-67-mix-Ir(III) (3)}

In a glovebox under inert atmosphere, to an oven-dried dram vial was added UiO-67-mix (437.1 $\mathrm{mg}, \sim 35 \%$ PhenDC incorporation) and $[\operatorname{Ir}(\mathrm{COD}) \mathrm{Cl}]_{2}(143.43 \mathrm{mg}, 0.214 \mathrm{mmol})$. To this was added anhydrous THF $(4.81 \mathrm{~mL})$ to create a suspension that was green. The vial was capped and shaken overnight to facilitate mass transport. The suspension changes from green to blue overnight. The suspension is brought outside of the glovebox and washed with THF through multiple cycles of centrifugation/decantation on benchtop. The color of the material changes from blue to orange. It is then left exposed to air overnight in the solid state. The next day, it is washed and soaked with acetone through multiple cycles of centrifugation/decantation. The material is then dried in a vacuum oven at $85^{\circ} \mathrm{C}$ for 1-2 hours after which it is activated under high vacuum on a Smart Vac Prep at $150{ }^{\circ} \mathrm{C}$ overnight. The brownish-orange material is then transferred without exposure to ambient atmosphere and stored in a glovebox under inert atmosphere.
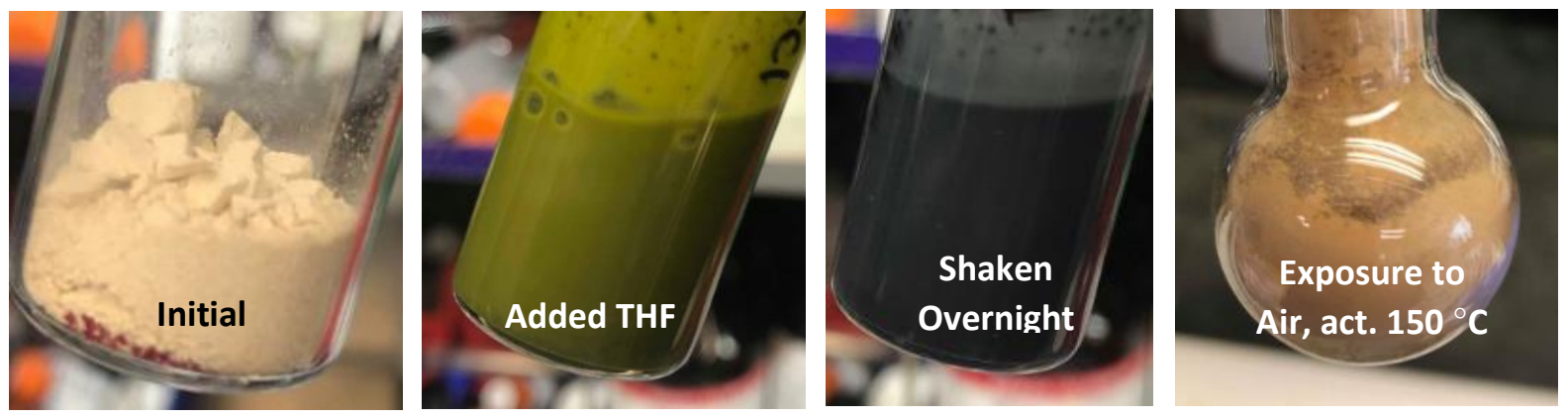

Figure S1. Color changes observed in metalation to synthesize 3. Initial represents solid mixture of white MOF and red $[\operatorname{Ir}(\mathrm{COD}) \mathrm{Cl}]_{2}$, after which a color change to green is observed when THF is added, after shaking overnight the suspension turns blue and after exposure to air and activation, a brownish-orange solid is obtained. 


\section{UiO-67-mix-Ir post-borylation (4)}

In a glovebox under inert atmosphere, to an oven-dried 2-5 $\mathrm{mL}$ microwave reaction vessel from Biotage $\AA$ was added $3(40 \mathrm{mg})$, anhydrous cyclohexane $(0.48 \mathrm{~mL})$, anhydrous benzene $(0.52$ $\mathrm{mL}, 5.8 \mathrm{mmol}$ ), and $\mathrm{B}_{2} \mathrm{Pin}_{2}$ as a stock solution in anhydrous cyclohexane (84 $\mathrm{mg}$ in $3 \mathrm{~mL}$ cyclohexane, syringe $2 \mathrm{~mL}$ into reaction, $56 \mathrm{mg}, 0.22 \mathrm{mmol})$. The reaction was sealed and placed on an aluminum block on a hotplate with thermocouple and the vial was wrapped in foil around the liquid meniscus for better insulation. It was stirred at $1000 \mathrm{rpm}$ for $280 \mathrm{~min}$ at $80{ }^{\circ} \mathrm{C}$. The reaction was then stopped and cooled to room temperature for $\sim 20 \mathrm{~min}$. After cooling, an aliquot was taken and analyzed by GC-FID. Inside the glovebox, the material was isolated via multiple cycles of centrifugation/decantation followed by multiple cycles of washing/soaking with pentane. The material was then activated under high vacuum on a Smart Vac Prep at $25^{\circ} \mathrm{C}$.

\section{UiO-67-mix-Ir post-borylation (4) for PDF/DED analysis}

The material was prepared according to a similar procedure to above. In a glovebox under inert atmosphere, to an oven-dried 2-5 mL microwave reaction vessel from Biotage® was added 3 (20 $\mathrm{mg})$, anhydrous cyclohexane, anhydrous benzene $(0.13 \mathrm{~mL}, 1.5 \mathrm{mmol}), \mathrm{B}_{2} \mathrm{Pin}_{2}$ as a stock solution in anhydrous cyclohexane $(28 \mathrm{mg}, 0.11 \mathrm{mmol})$, and anhydrous hexadecane $(10 \mu \mathrm{L}, 0.034 \mathrm{mmol})$ as the internal standard. The reaction was sealed and placed on an aluminum block on a hotplate with thermocouple and the vial was wrapped in foil around the liquid meniscus for better insulation. It was stirred at $1000 \mathrm{rpm}$ for 3 hours at $80^{\circ} \mathrm{C}$. The reaction was then stopped, and an aliquot was taken and analyzed by GC-FID. It was cooled to room temperature and the supernatant was decanted. More anhydrous cyclohexane was added. The material was allowed to settle, and it was washed with copious amounts of anhydrous cyclohexane and anhydrous pentane. The material was then activated under vacuum at room temperature.

\section{UiO-67-mix-Ir post-borylation (4) for XAS}

The material was prepared according to a similar procedure to above. Note, the native MOF 1 was prepared using ultra-pure zirconyl chloride hydrate without hafnium impurity for the catalyst batch 3 that was used for this preparation. In a glovebox under inert atmosphere, to an oven-dried $20 \mathrm{~mL}$ glass scintillation vial was added 3 (40 mg), $\mathrm{B}_{2} \mathrm{Pin}_{2}(112 \mathrm{mg}, 0.44 \mathrm{mmol}$ ), anhydrous cyclohexane $(6 \mathrm{~mL})$, and anhydrous benzene $(0.26 \mathrm{~mL}, 2.9 \mathrm{mmol})$. The reaction was sealed and placed on an aluminum block on a hotplate with thermocouple. It was shaken at $65{ }^{\circ} \mathrm{C}$ for 23 hours. The reaction was then stopped, and filtered under vacuum in the glovebox, washing with copious amounts of pentane. It was then dried overnight under vacuum overnight.

\section{(Phen) $\operatorname{Ir}(\mathrm{COD})(\mathrm{Cl})$}

(Phen) $\operatorname{Ir}(\mathrm{COD})(\mathrm{Cl})$ was prepared according to previously reported procedures. ${ }^{2}$ 


\section{Characterization of Materials}

\section{${ }^{1}$ H NMR Spectra}

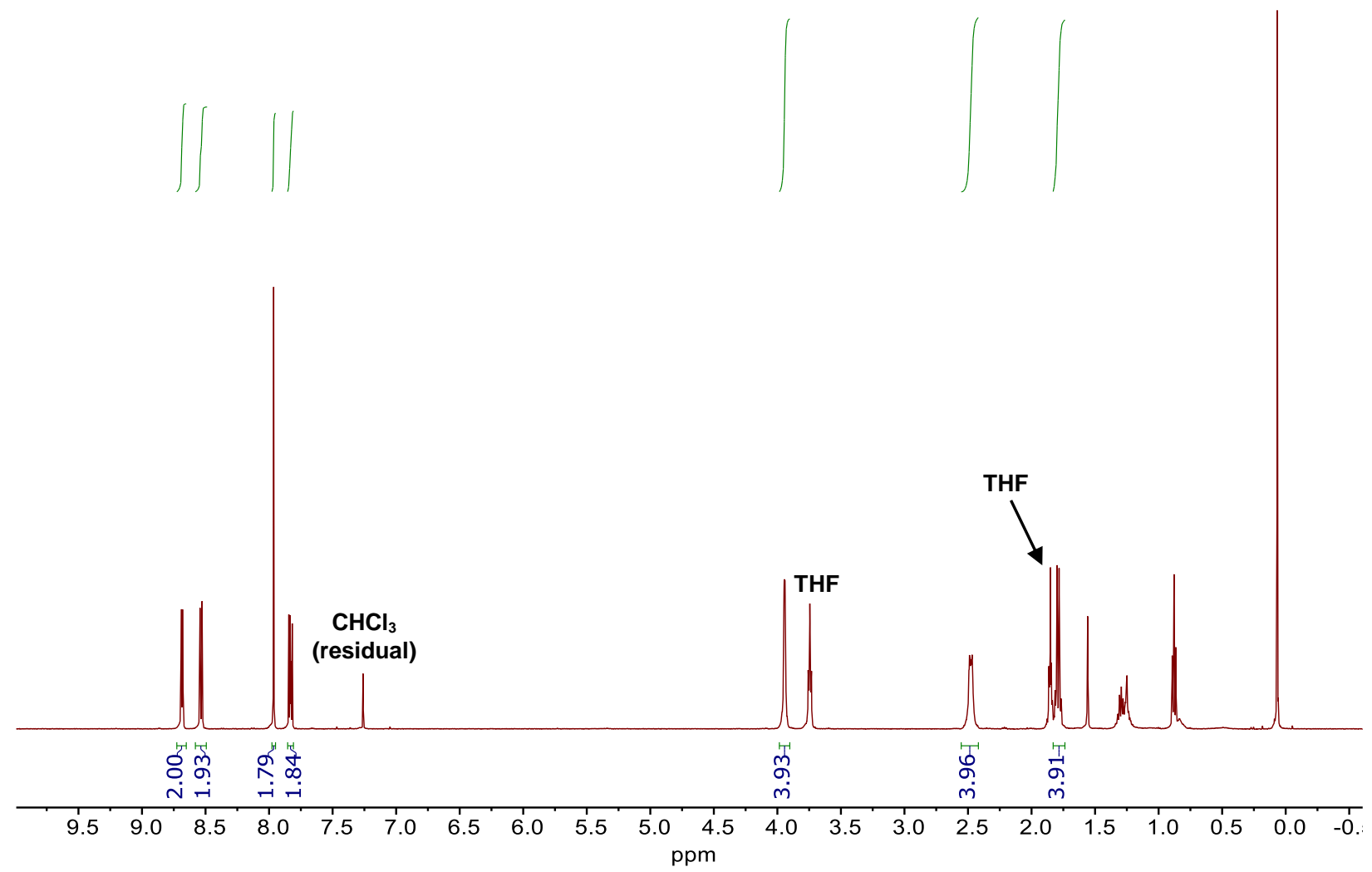

Figure S2. ${ }^{1} \mathrm{H}$ NMR spectrum of (Phen) $\operatorname{Ir}(\mathrm{COD})(\mathrm{Cl})$ in $\mathrm{CDCl}_{3}$ synthesized according to previously published procedures. ${ }^{2}$ 


\section{Powder XRD Patterns}

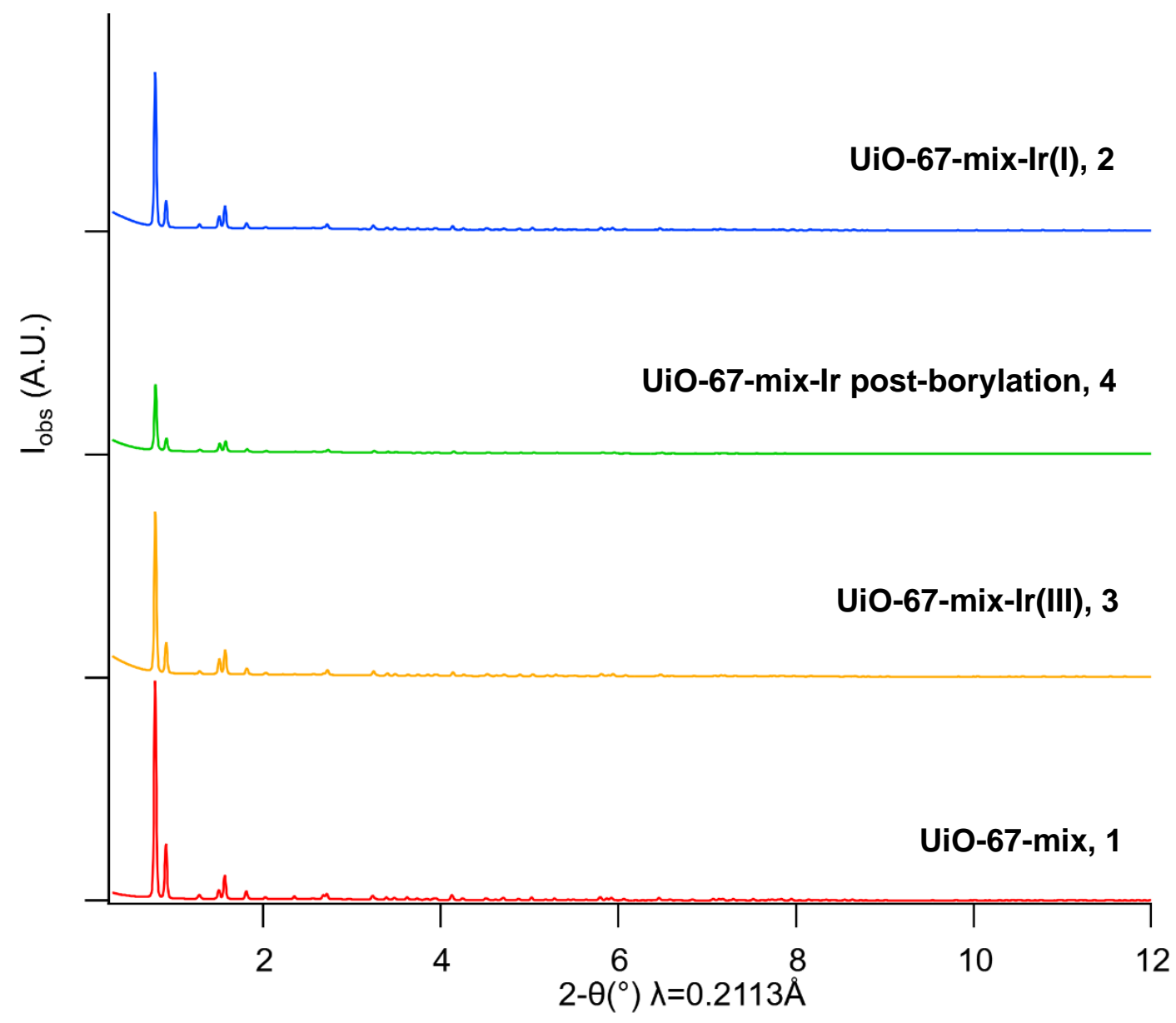

Figure S3. Powder XRD patterns of 1, 2, 3, 4 showing that crystallinity and phase purity is maintained after treatments. 


\section{$\mathrm{N}_{2}$ Sorption Data}

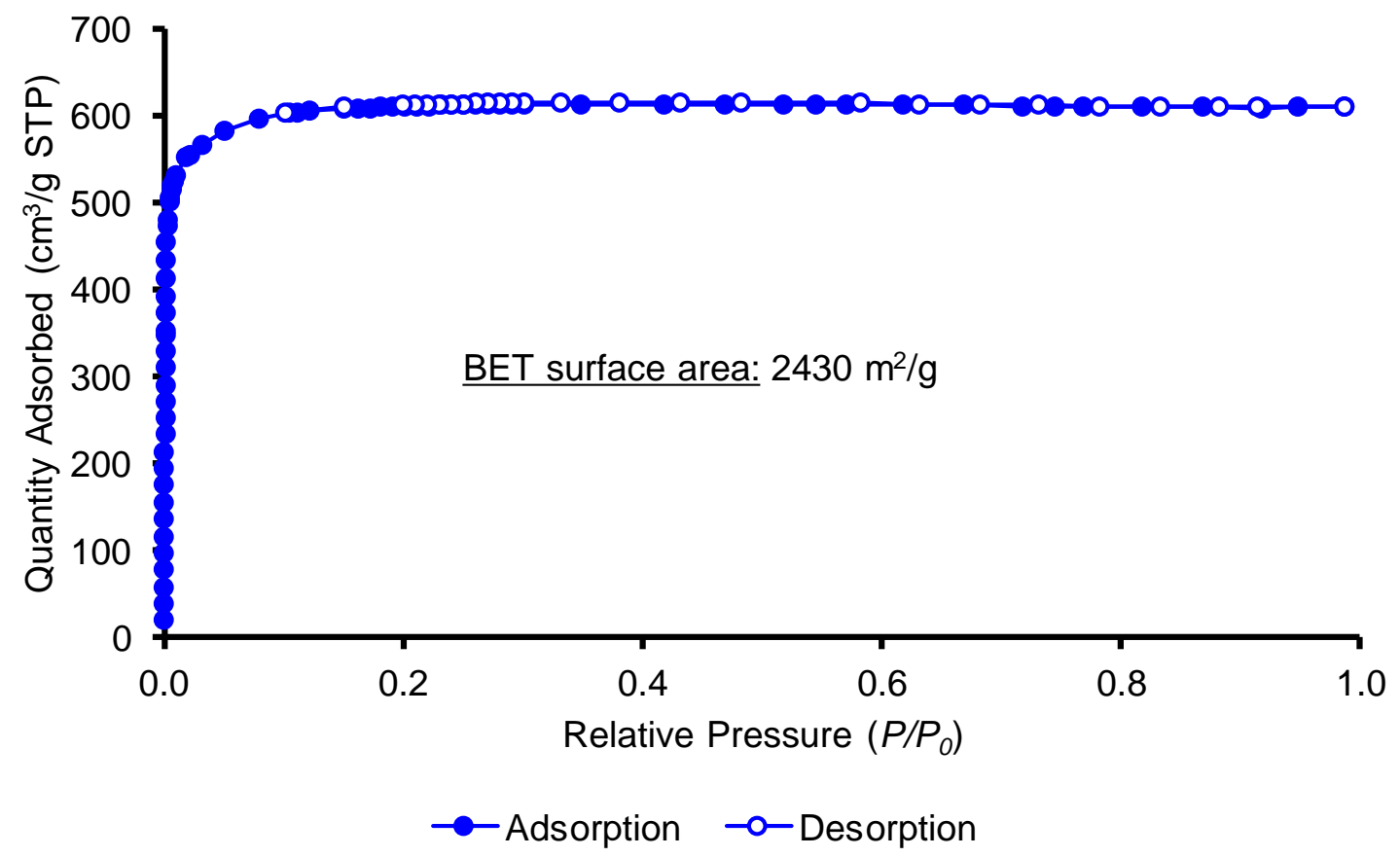

Figure S4. $\mathrm{N}_{2}$ adsorption/desorption isotherm of UiO-67-mix (1) collected at $77 \mathrm{~K}$.

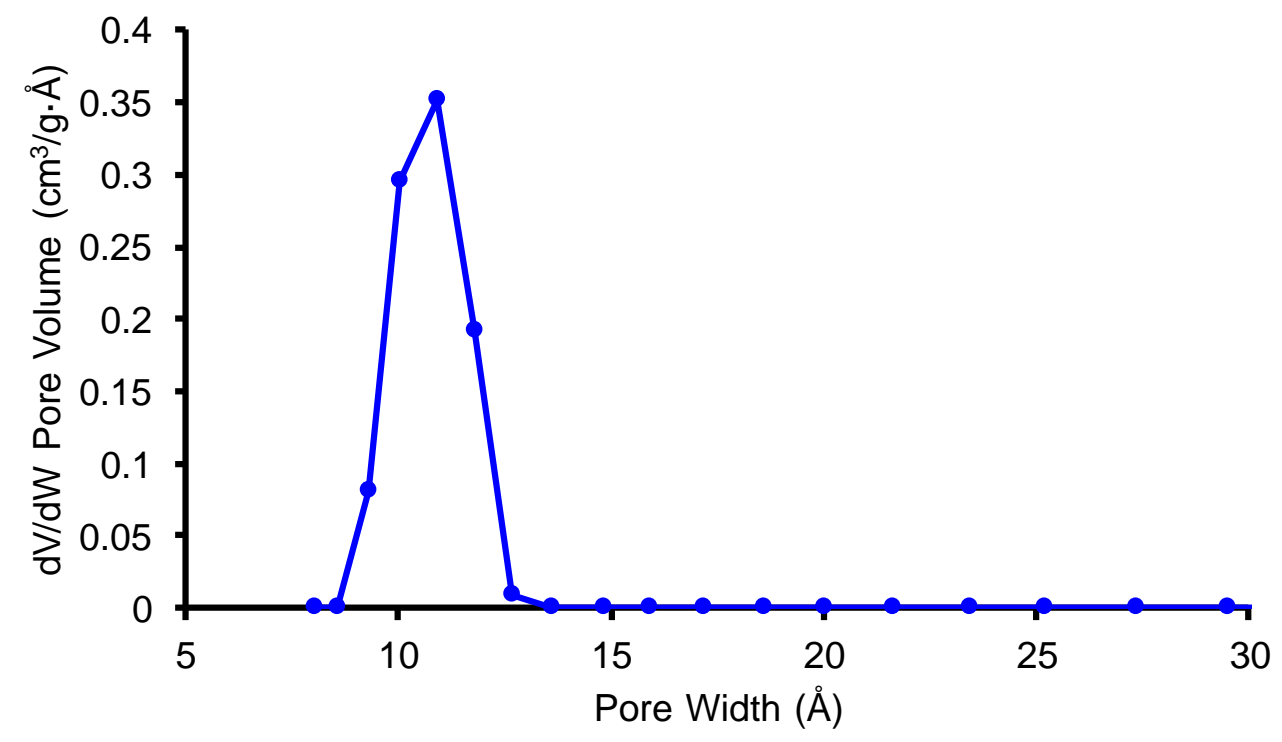

Figure S5. DFT Pore Size Distribution of UiO-67-mix (1) collected at $77 \mathrm{~K}$. 


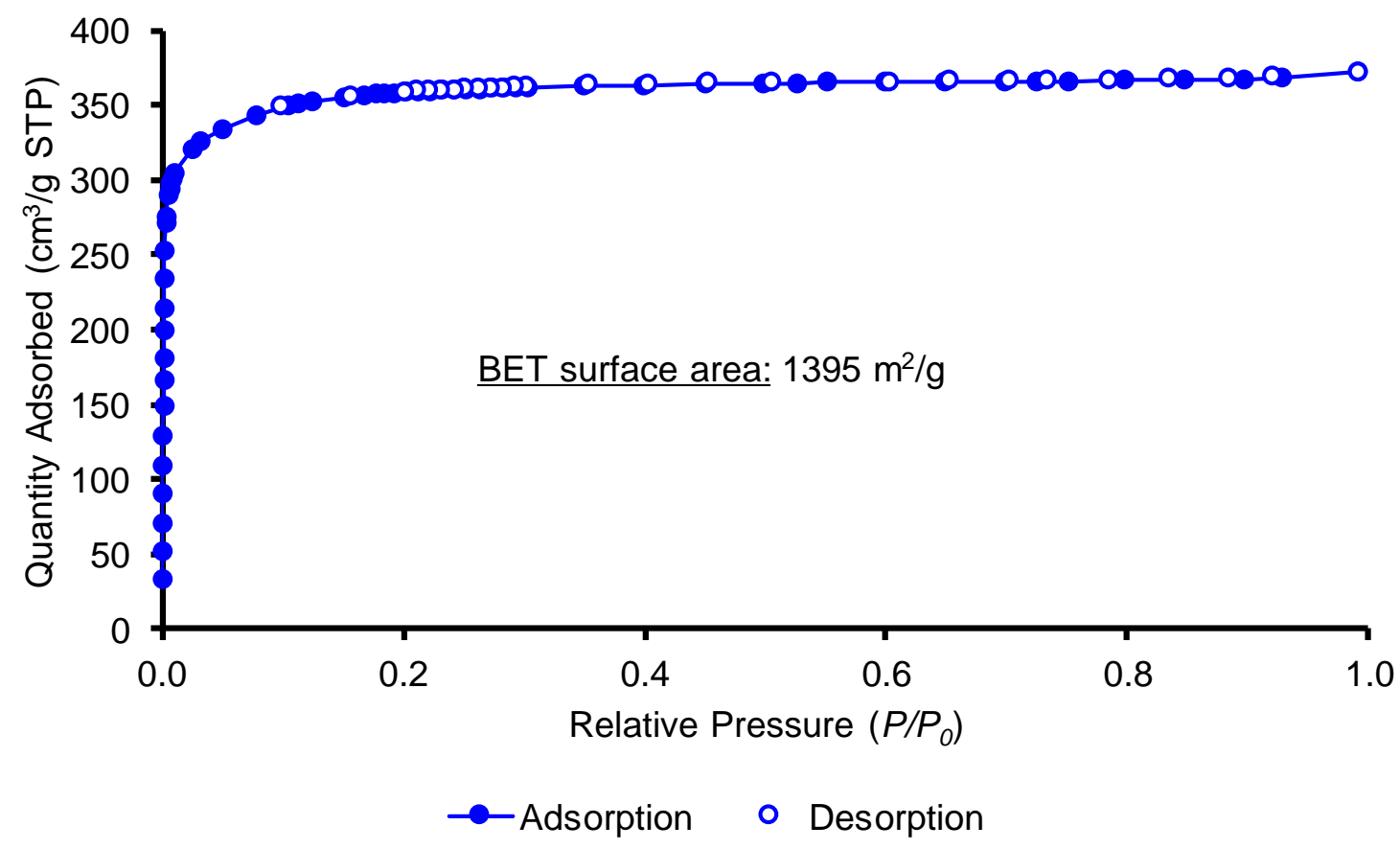

Figure S6. $\mathrm{N}_{2}$ adsorption/desorption isotherm of UiO-67-mix-Ir(I) (2) collected at $77 \mathrm{~K}$.

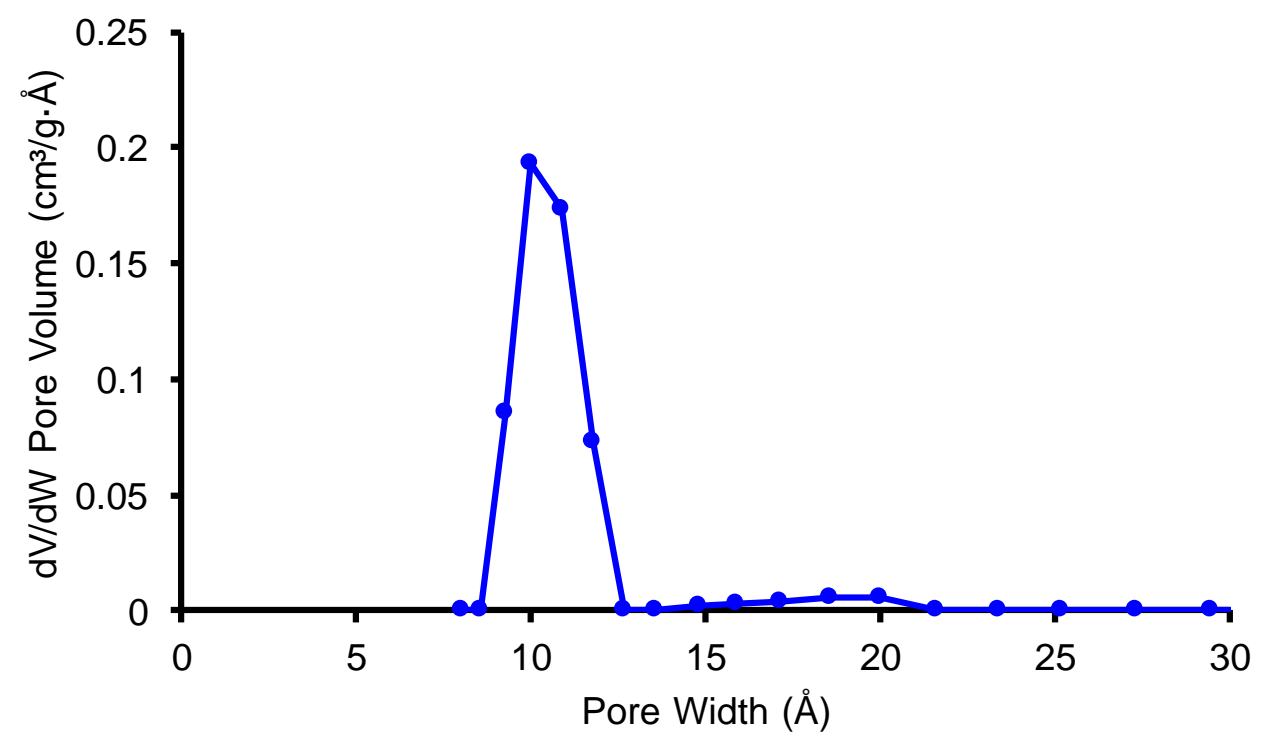

Figure S7. DFT Pore Size Distribution of UiO-67-mix-Ir(I) (2) collected at $77 \mathrm{~K}$. 


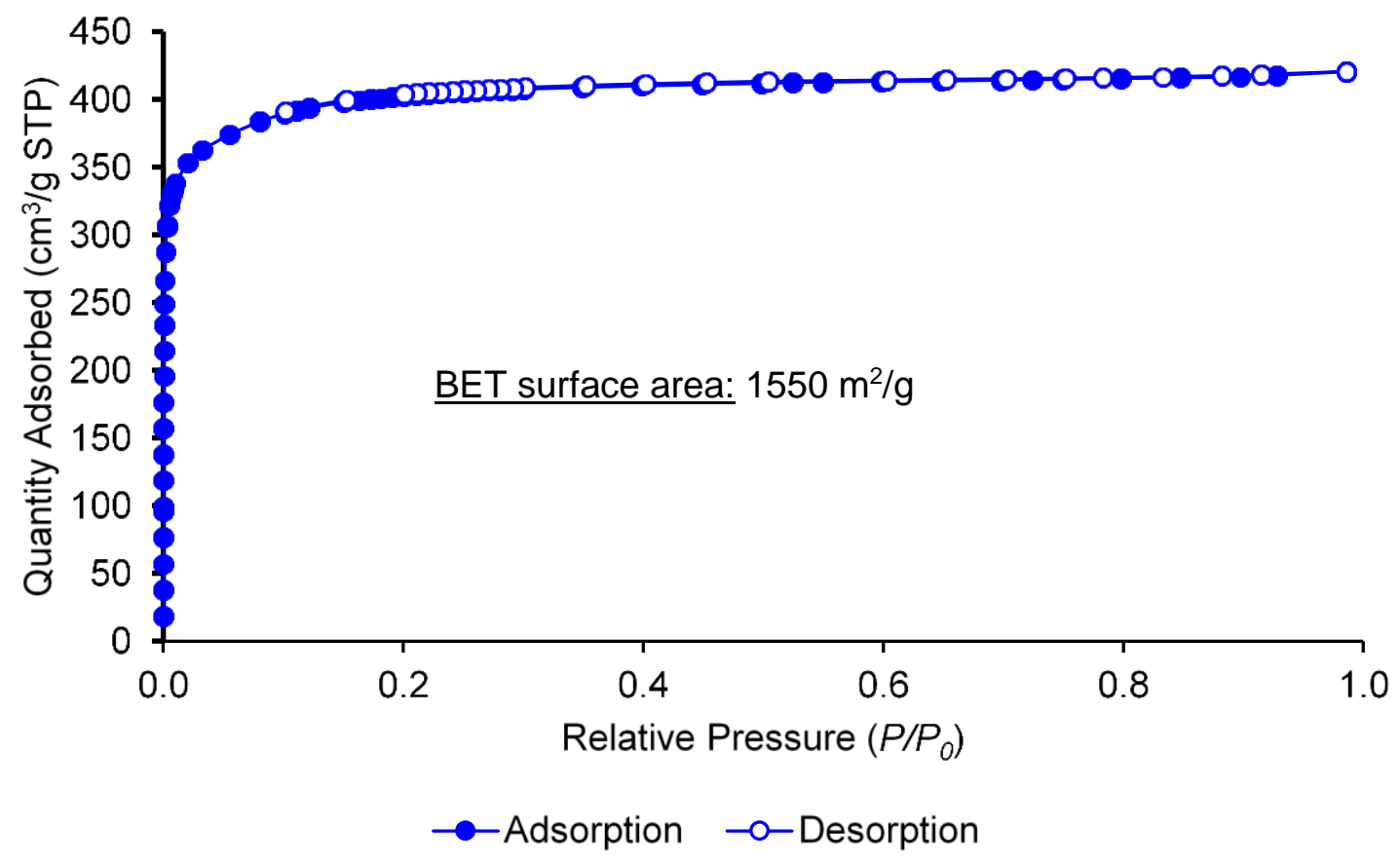

Figure S8. $\mathrm{N}_{2}$ adsorption/desorption isotherm of UiO-67-mix-Ir(III) (3) collected at $77 \mathrm{~K}$.

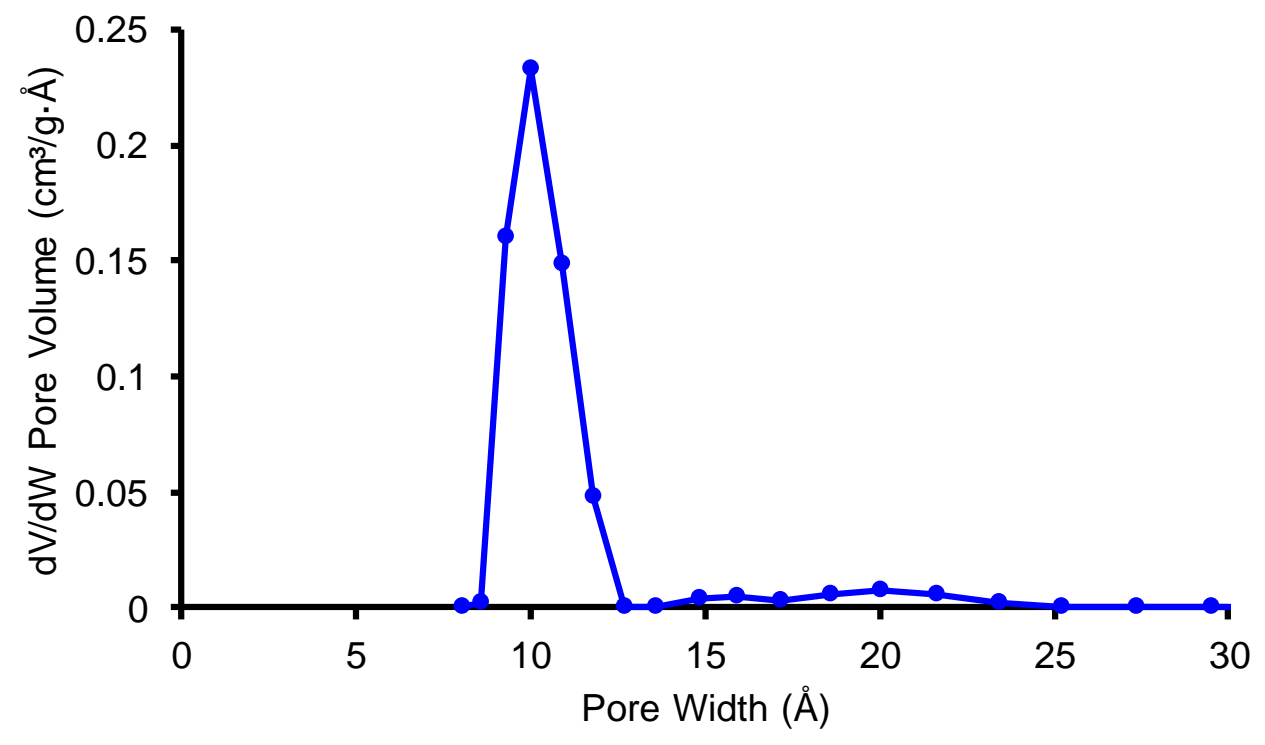

Figure S9. DFT Pore Size Distribution of UiO-67-mix-Ir(III) (3) collected at $77 \mathrm{~K}$. 


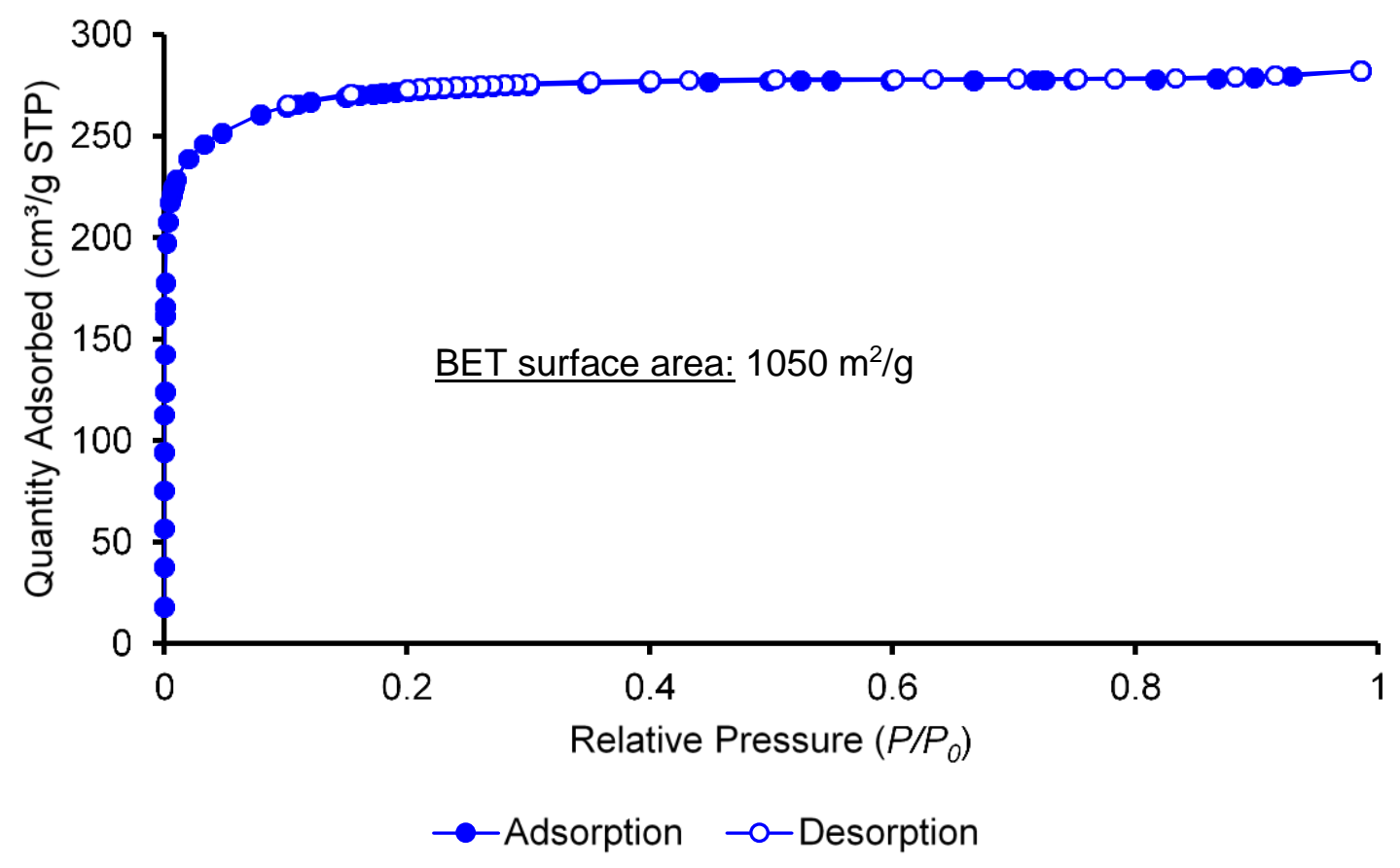

Figure S10. $\mathrm{N}_{2}$ adsorption/desorption isotherm of UiO-67-mix-Ir post-borylation (4) collected at $77 \mathrm{~K}$.

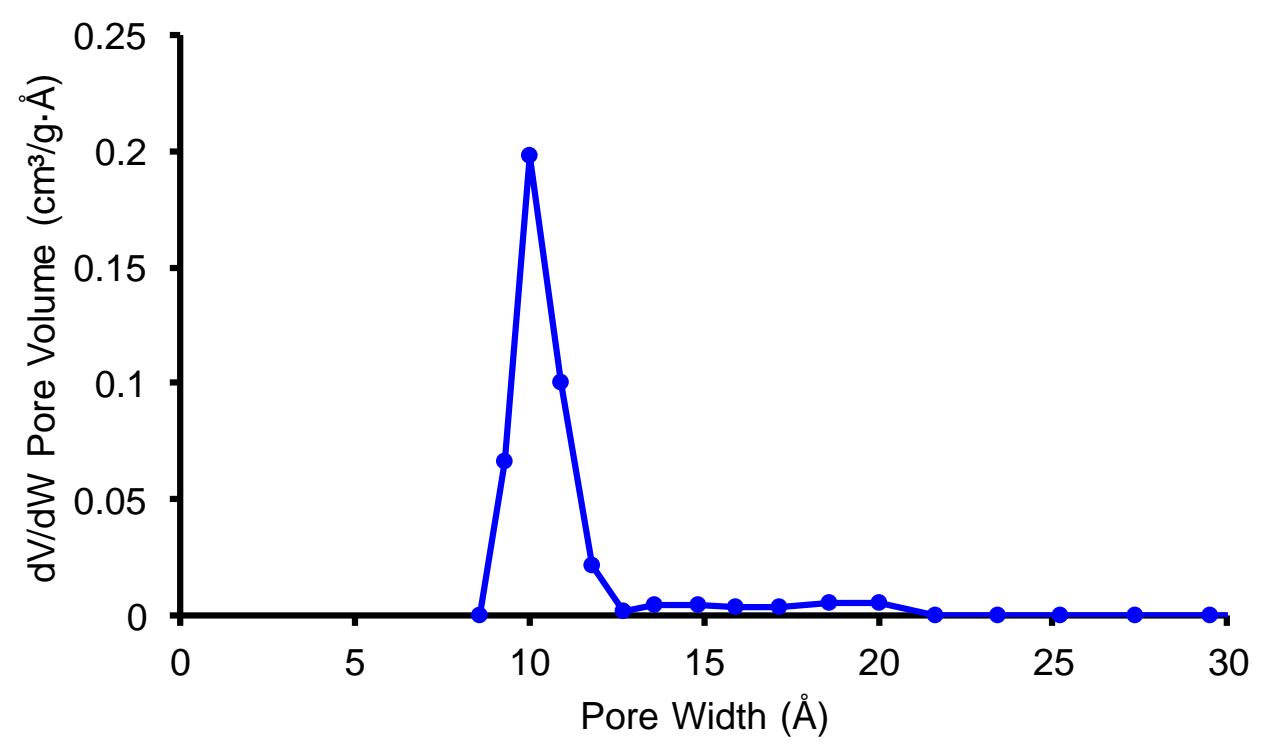

Figure S11. DFT Pore Size Distribution of UiO-67-mix-Ir post-borylation (4) collected at $77 \mathrm{~K}$. 


\section{SEM Images}

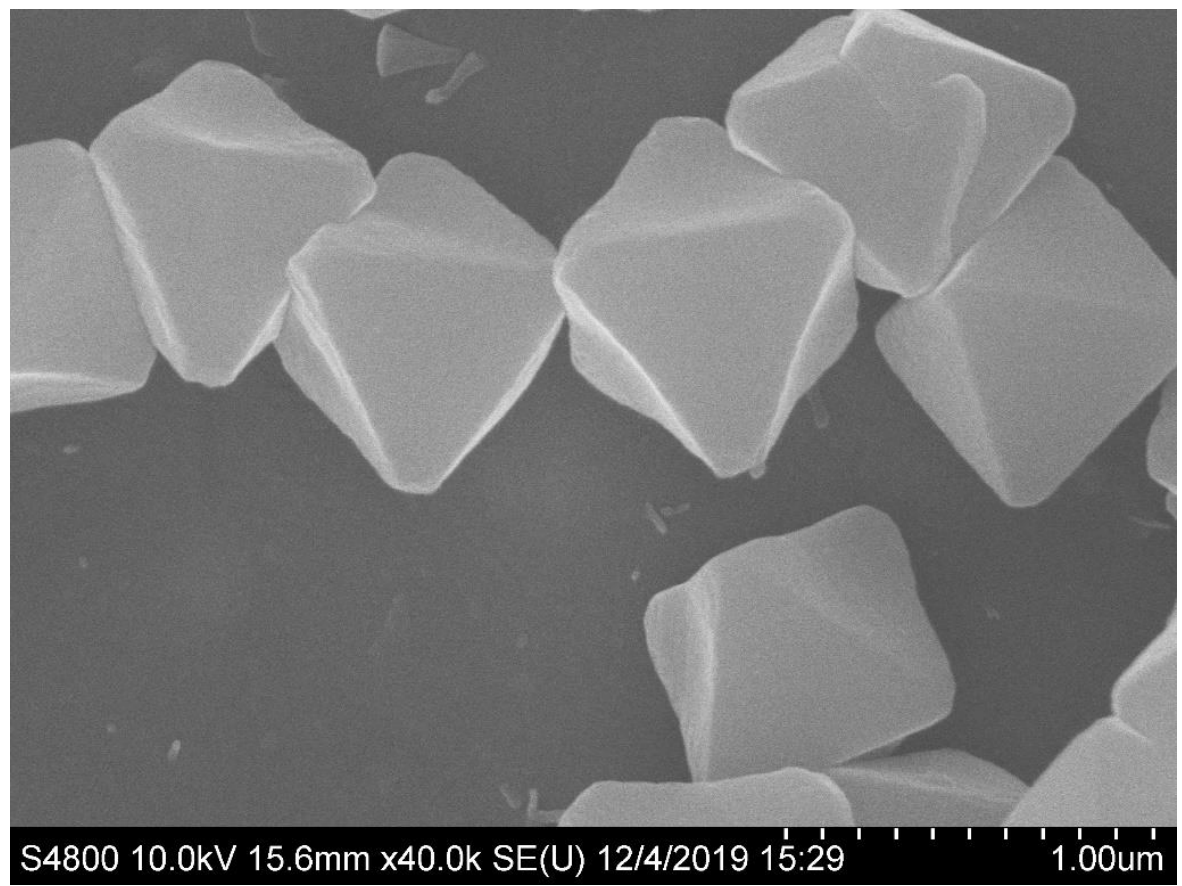

Figure S12. SEM image of UiO-67-mix-Ir(III) (3).

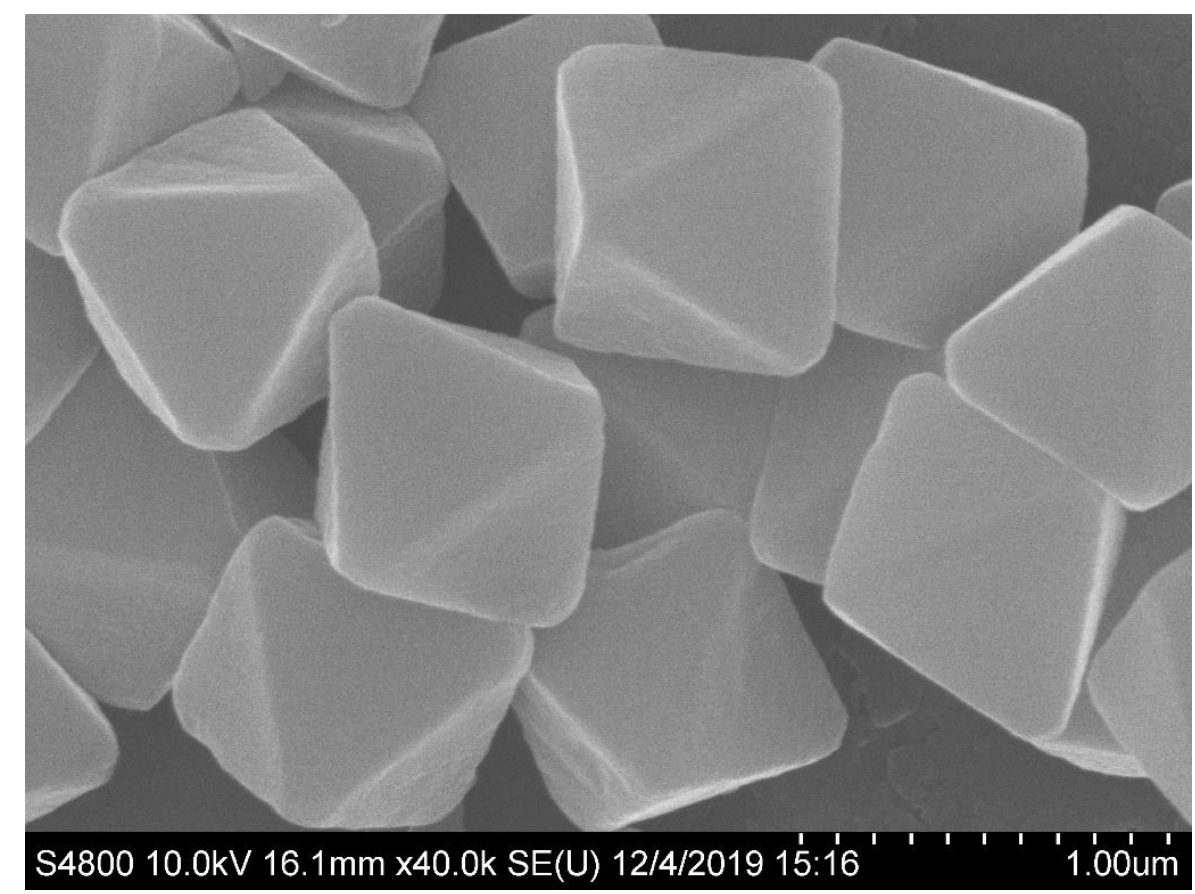

Figure S13. SEM image of UiO-67-mix-Ir post-borylation (4). 


\section{DRIFTS Spectra}
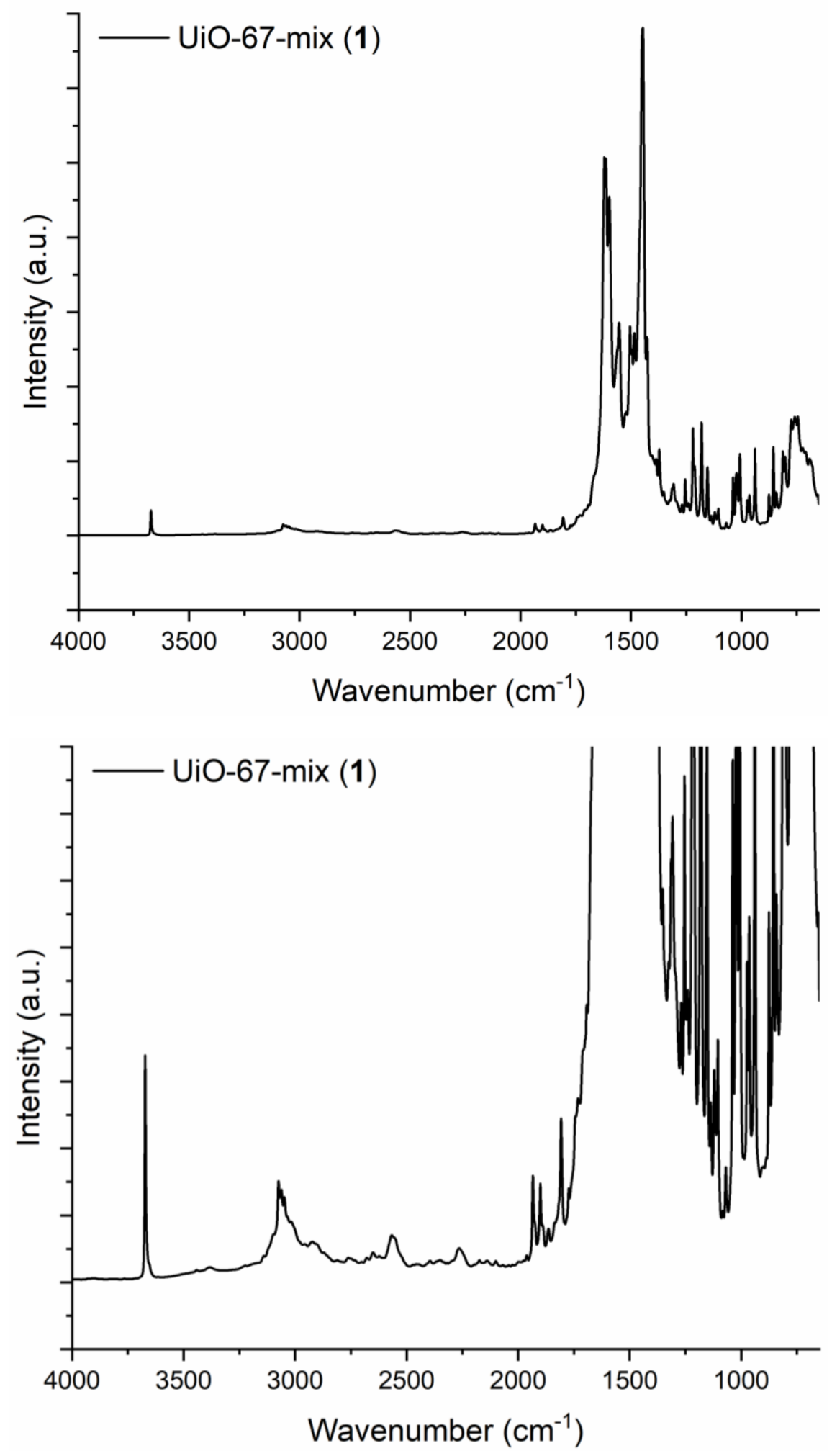

Figure S14. DRIFTS of UiO-67-mix (1), full spectrum (top), zoomed-up (bottom). 

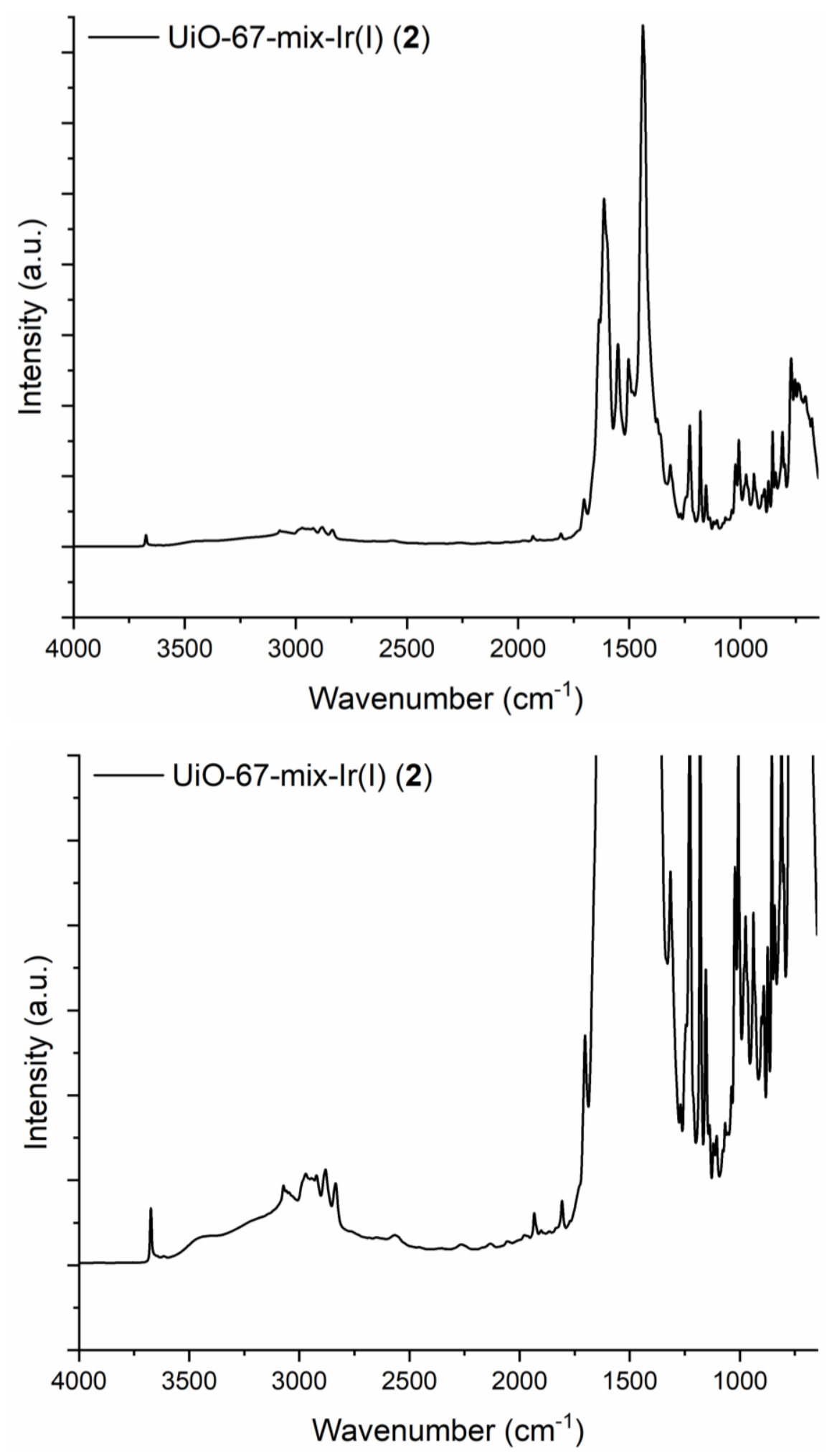

Figure S15. DRIFTS of UiO-67-mix-Ir(I) (2), full spectrum (top), zoomed-up (bottom). 

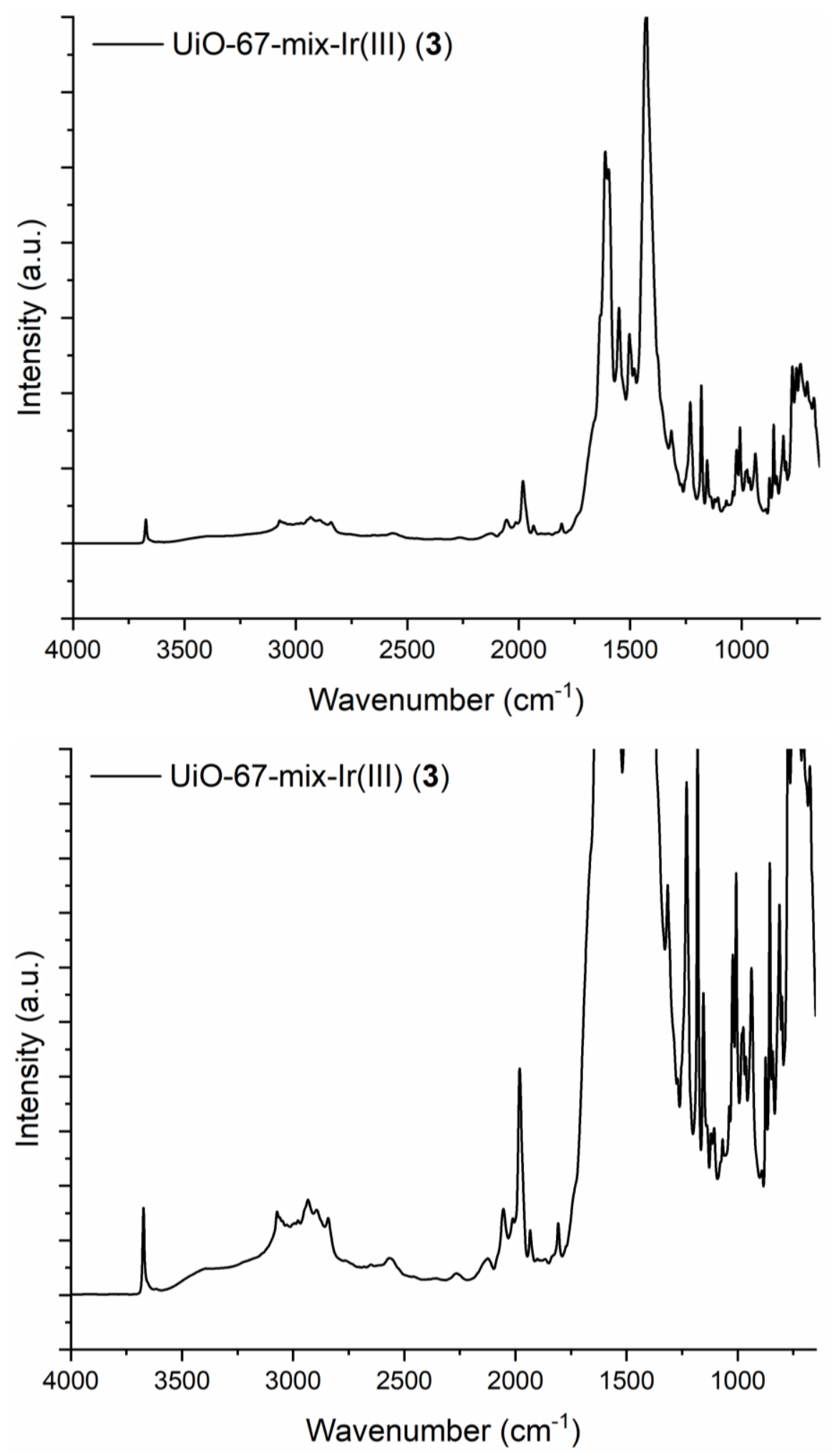

Figure S16. DRIFTS of UiO-67-mix-Ir(III) (3), full spectrum (top), zoomed-up (bottom). 


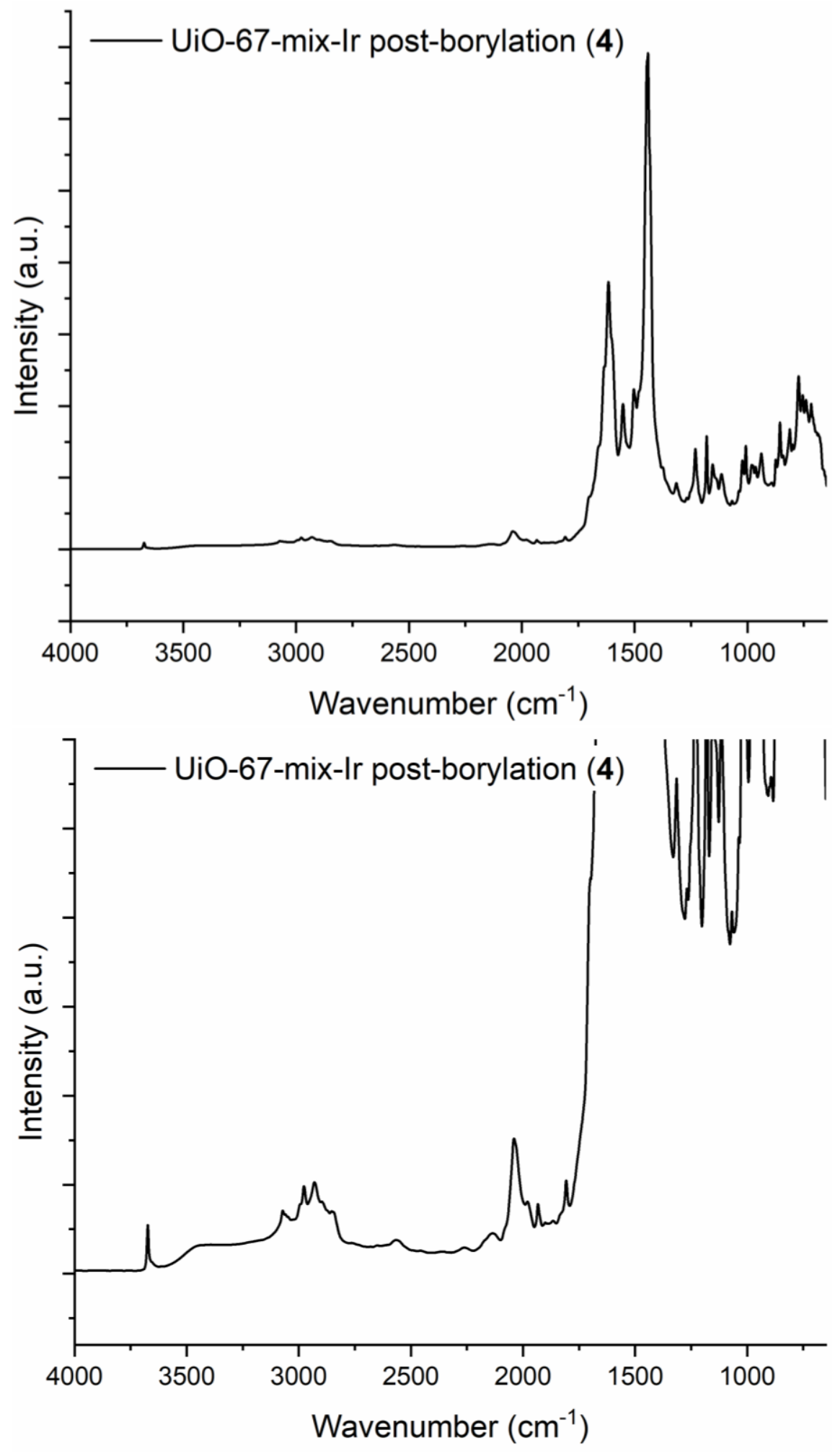

Figure S17. DRIFTS of UiO-67-mix-Ir post-borylation (4), full spectrum (top), zoomed-up (bottom). 


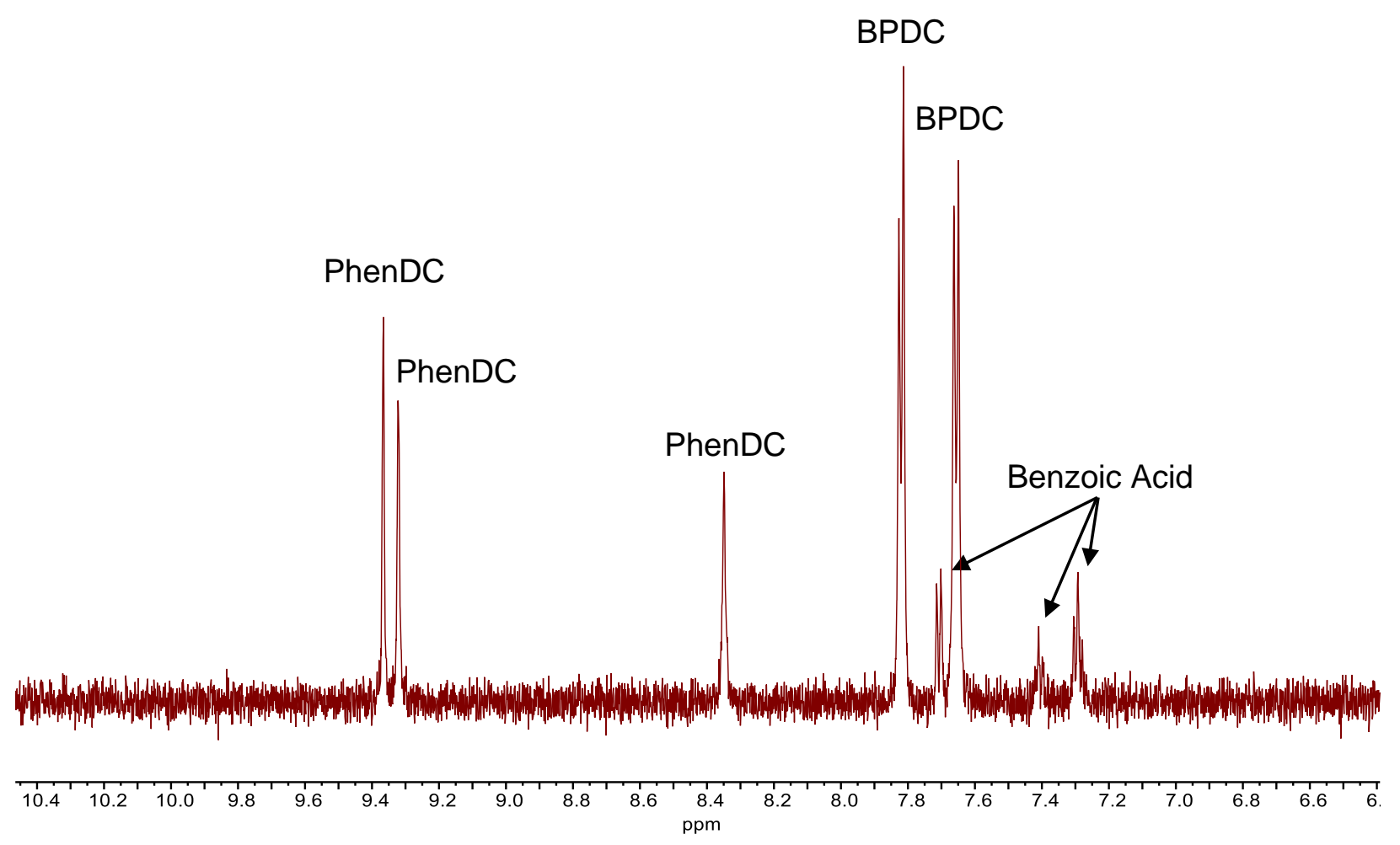

Figure S18. ${ }^{1} \mathrm{H}$ NMR acid digestion of UiO-67-mix $\left(\mathrm{D}_{2} \mathrm{SO}_{4} / \mathrm{DMSO}-d_{6}\right)$

ICP data

UiO-67-mix-Ir(III) (3)

Table S1. Average concentrations of acid-digested ICP sample of 3

\begin{tabular}{|c|c|c|c|c|c|}
\hline Element & $\begin{array}{c}\text { Average } \\
\text { concentration } \\
1\end{array}$ & $\begin{array}{c}\text { Average } \\
\text { concentration } \\
2\end{array}$ & $\begin{array}{c}\text { Average } \\
\text { concentration } \\
3\end{array}$ & $\begin{array}{c}\text { Average } \\
\text { concentration } \\
4\end{array}$ & Units \\
\hline $\mathrm{Zr}$ & 6.548 & 6.813 & 6.082 & 6.246 & $\mathrm{ppm}$ \\
\hline Ir & 5.118 & 4.746 & 4.606 & 4.946 & ppm \\
\hline
\end{tabular}

Table S2. Calculated analyte concentrations and weight percent based on Table S1.

\begin{tabular}{|c|c|c|c|c|c|c|c|}
\hline $\begin{array}{c}\text { Mass of } \\
\text { Sample } \\
\text { Digested } \\
(\mathrm{mg})\end{array}$ & $\begin{array}{l}\text { Mass in } \\
\text { ICP } \\
\text { sample } \\
(\mathrm{mg})\end{array}$ & $\begin{array}{l}\text { Concentration } \\
\operatorname{Zr}(p p m)\end{array}$ & $\begin{array}{l}\text { Concentration } \\
\text { Ir (ppm) }\end{array}$ & $\begin{array}{c}\text { mmol Zr } \\
\text { in ICP } \\
\text { sample }\end{array}$ & $\begin{array}{c}\text { mmol Ir in } \\
\text { ICP } \\
\text { sample }\end{array}$ & $\mathbf{I r} / \mathbf{Z r}_{6}$ & $\begin{array}{l}\% \text { Ir (by } \\
\text { weight) }\end{array}$ \\
\hline 2.5 & 0.375 & 6.4 & 4.9 & 0.000704 & 0.0002525 & 2.15 & 12.94 \\
\hline
\end{tabular}


Table S3. Average concentrations of acid-digested (quartz vessel) ICP sample of 3

\begin{tabular}{c|ccccc} 
Element & $\begin{array}{c}\text { Average } \\
\text { concentration } \\
\mathbf{1}\end{array}$ & $\begin{array}{c}\text { Average } \\
\text { concentration } \\
\mathbf{2}\end{array}$ & $\begin{array}{c}\text { Average } \\
\text { concentration } \\
\mathbf{3}\end{array}$ & $\begin{array}{c}\text { Average } \\
\text { concentration } \\
\mathbf{4}\end{array}$ & Units \\
\hline $\mathrm{Zr}$ & 7.035 & 7.163 & 7.165 & 7.039 & $\mathrm{ppm}$ \\
$\mathrm{Ir}$ & 5.481 & 5.526 & 5.591 & 5.78 & $\mathrm{ppm}$ \\
$\mathrm{B}$ & 0.055 & 0.061 & 0.044 & - & $\mathrm{ppm}$
\end{tabular}

Table S4. Calculated analyte concentrations based on Table S3.

\begin{tabular}{ccccc}
$\begin{array}{c}\text { Concentration } \\
\text { B (ppm) }\end{array}$ & $\begin{array}{c}\text { Concentration Ir } \\
\text { (ppm) }\end{array}$ & $\begin{array}{c}\text { mmol B in ICP } \\
\text { sample }\end{array}$ & $\begin{array}{c}\text { mmol Ir in ICP } \\
\text { sample }\end{array}$ & B/Ir \\
\hline 0.05 & 5.6 & 0.000049 & 0.0002911 & 0.17
\end{tabular}

UiO-67-mix-Ir post-borylation (4)

Table S5. Average concentrations of acid-digested ICP sample of 4

\begin{tabular}{c|ccccc} 
Element & $\begin{array}{c}\text { Average } \\
\text { concentration } \\
\mathbf{1}\end{array}$ & $\begin{array}{c}\text { Average } \\
\text { concentration } \\
\mathbf{2}\end{array}$ & $\begin{array}{c}\text { Average } \\
\text { concentration } \\
\mathbf{3}\end{array}$ & $\begin{array}{c}\text { Average } \\
\text { concentration } \\
\mathbf{4}\end{array}$ & Units \\
\hline $\mathrm{Zr}$ & 5.891 & 6.094 & 6.091 & 5.826 & $\mathrm{ppm}$ \\
$\mathrm{Ir}$ & 4.584 & 4.675 & 4.312 & 4.767 & $\mathrm{ppm}$ \\
$\mathrm{B}$ & 0.147 & 0.164 & 0.144 & - & $\mathrm{ppm}$
\end{tabular}

Table S6. Calculated analyte concentrations and weight percent based on Table S5.

\begin{tabular}{|c|c|c|c|c|c|c|c|}
\hline $\begin{array}{c}\text { Mass of } \\
\text { Sample } \\
\text { Digested } \\
(\mathrm{mg})\end{array}$ & $\begin{array}{l}\text { Mass in } \\
\text { ICP } \\
\text { sample } \\
(\mathrm{mg})\end{array}$ & $\begin{array}{c}\text { Concentration } \\
\operatorname{Zr}(p p m)\end{array}$ & $\begin{array}{l}\text { Concentration } \\
\operatorname{Ir}(\mathrm{ppm})\end{array}$ & $\begin{array}{c}\text { mmol Zr } \\
\text { in ICP } \\
\text { sample }\end{array}$ & $\begin{array}{c}\text { mmol Ir in } \\
\text { ICP } \\
\text { sample }\end{array}$ & $\mathbf{I r} / \mathbf{Z r}_{6}$ & $\begin{array}{l}\text { \%lr (by } \\
\text { weight) }\end{array}$ \\
\hline 2.3 & 0.339 & 6.0 & 4.6 & 0.000655 & 0.0002385 & 2.18 & 13.52 \\
\hline
\end{tabular}

Table S7. Calculated analyte concentrations based on Table S5.

\begin{tabular}{ccccc}
$\begin{array}{c}\text { Concentration B } \\
(\mathbf{p p m})\end{array}$ & $\begin{array}{c}\text { Concentration Ir } \\
(\mathbf{p p m})\end{array}$ & $\begin{array}{c}\text { mmol B in ICP } \\
\text { sample }\end{array}$ & $\begin{array}{c}\text { mmol Ir in ICP } \\
\text { sample }\end{array}$ & B/Ir \\
\hline 0.15 & 4.6 & 0.000140 & 0.0002385 & 0.59
\end{tabular}




\section{XAS data}

XANES for 3, $[\operatorname{Ir}(\mathrm{COD}) \mathrm{Cl}]_{2}$, and $(\mathrm{Phen}) \operatorname{Ir}(\mathrm{COD})(\mathrm{Cl})$

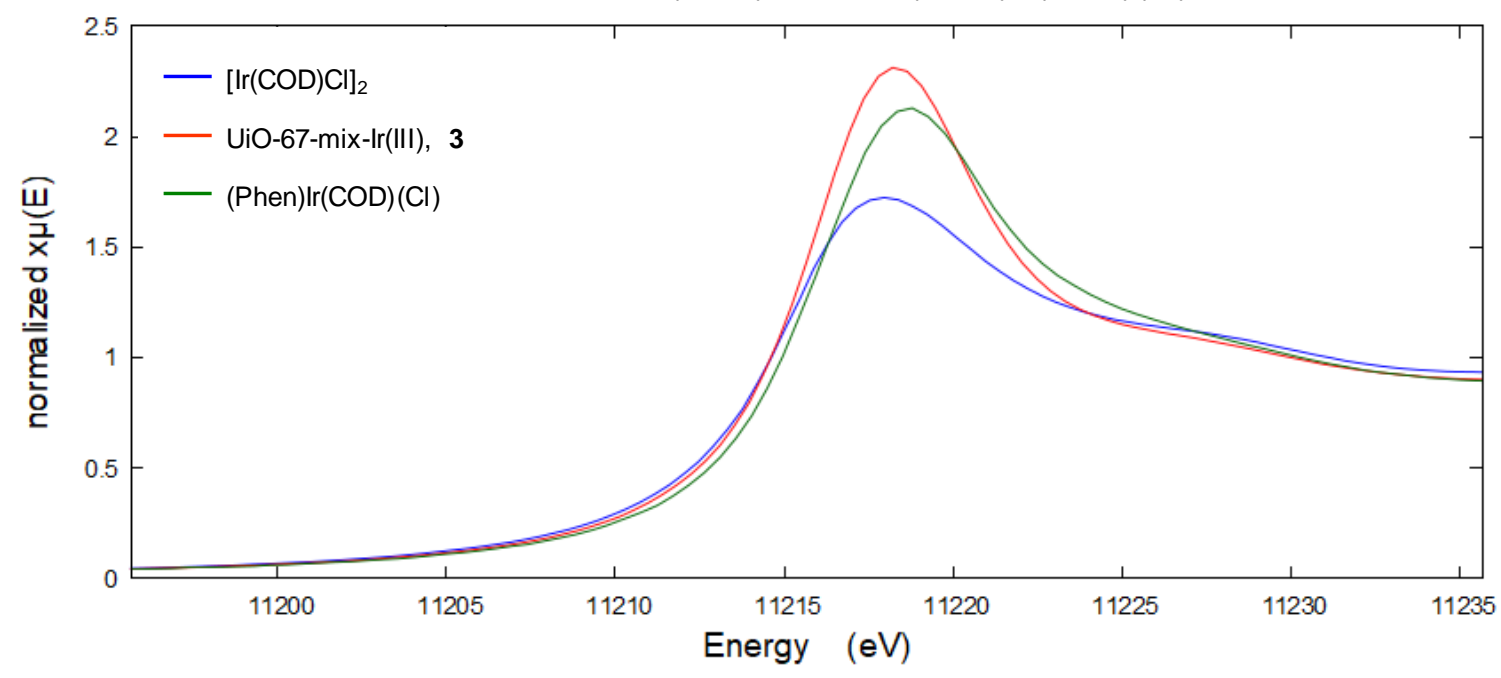

Figure S19. Ir L3 edge XANES of UiO-67-mix-Ir(III), 3, $[\operatorname{Ir}(\mathrm{COD}) \mathrm{Cl}]_{2}$ and (Phen) $\operatorname{Ir}(\mathrm{COD})(\mathrm{Cl})$.

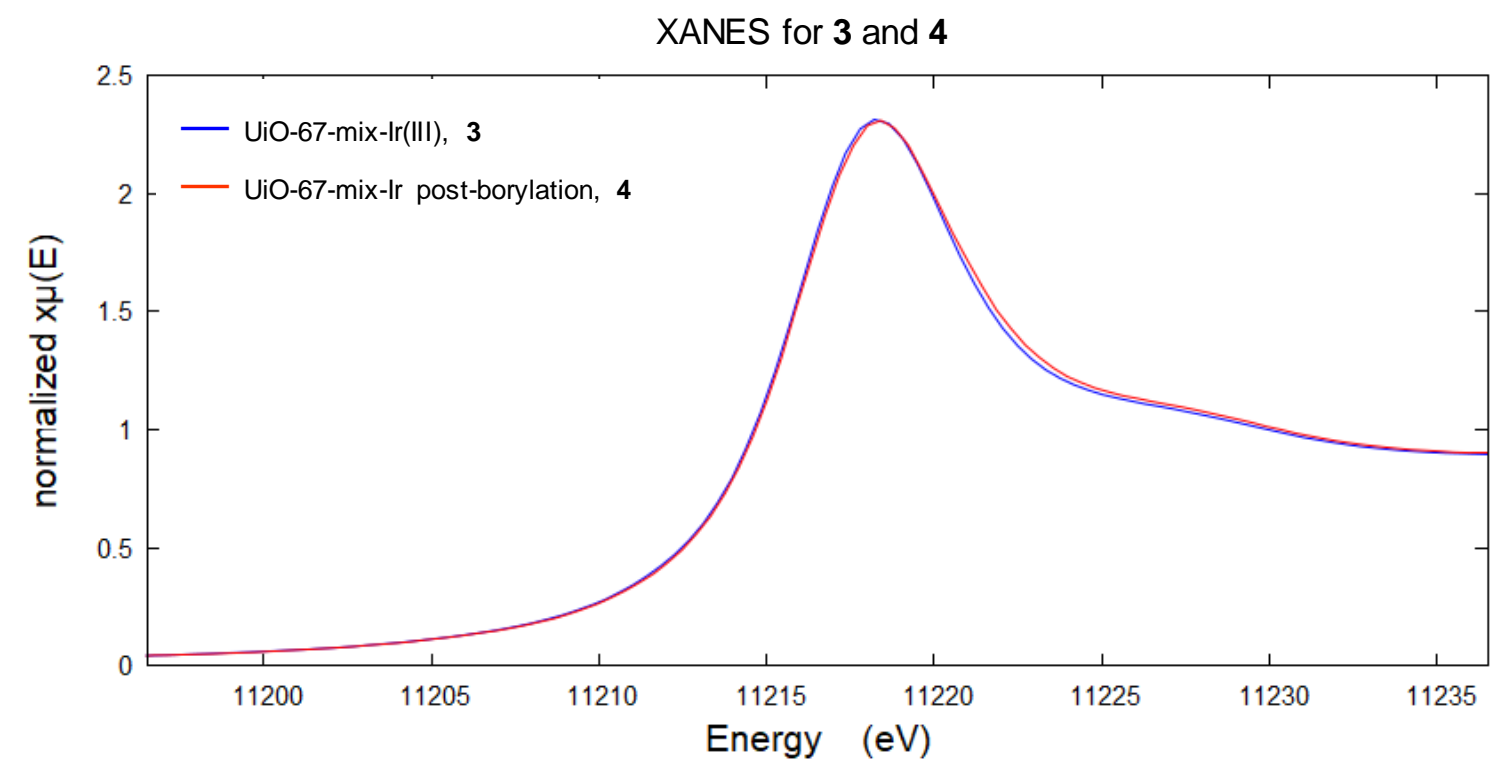

Figure S20. Ir L3 edge XANES of UiO-67-mix-Ir(III), 3, and UiO-67-mix-Ir post-borylation, 4. 


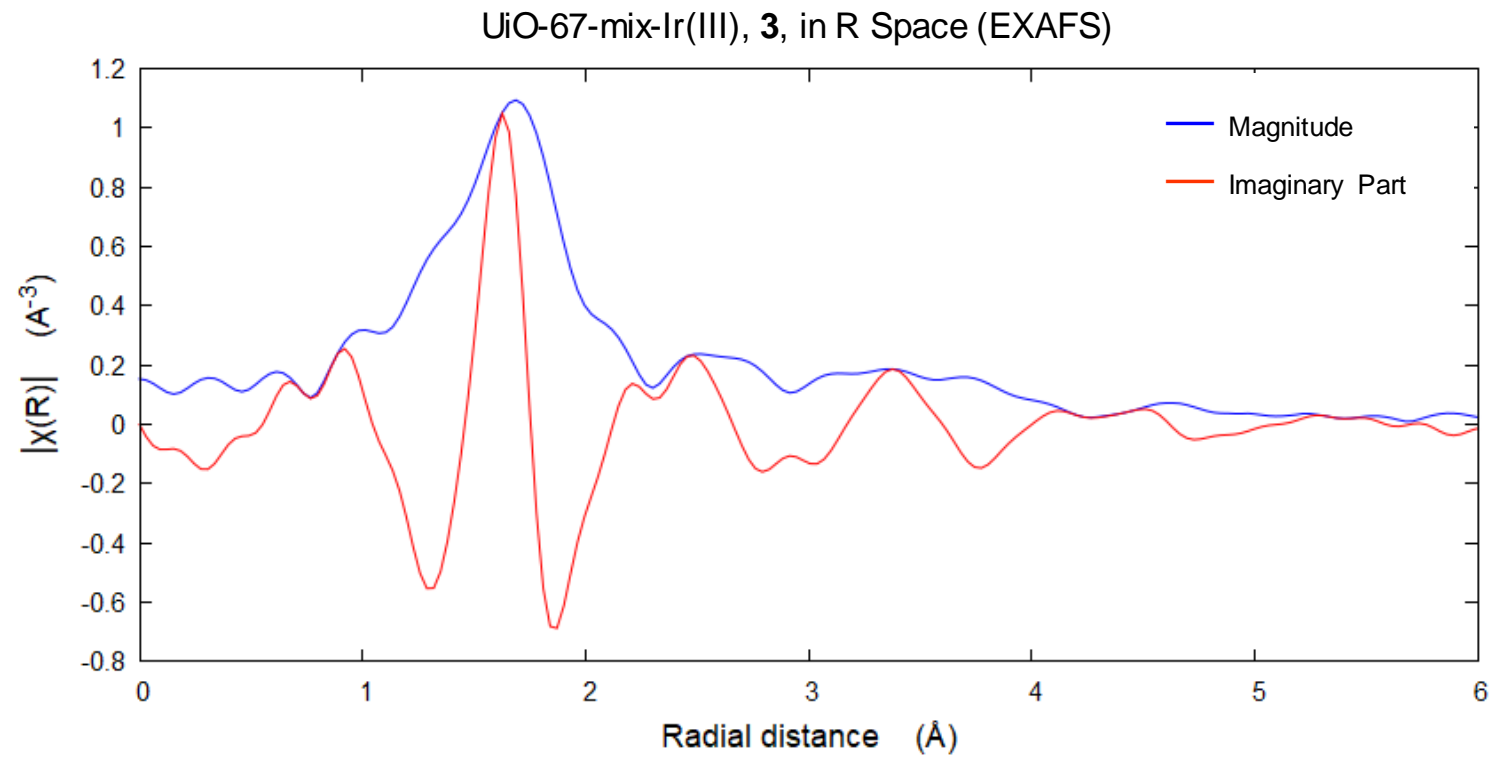

Figure S21. The magnitude and imaginary part of the $k^{2}$-weighted EXAFS of UiO-67-mix-Ir(III), 3.

UiO-67-mix-Ir(III), 3, and $[\operatorname{Ir}(\mathrm{COD}) \mathrm{Cl}]_{2}$ in R Space (EXAFS)

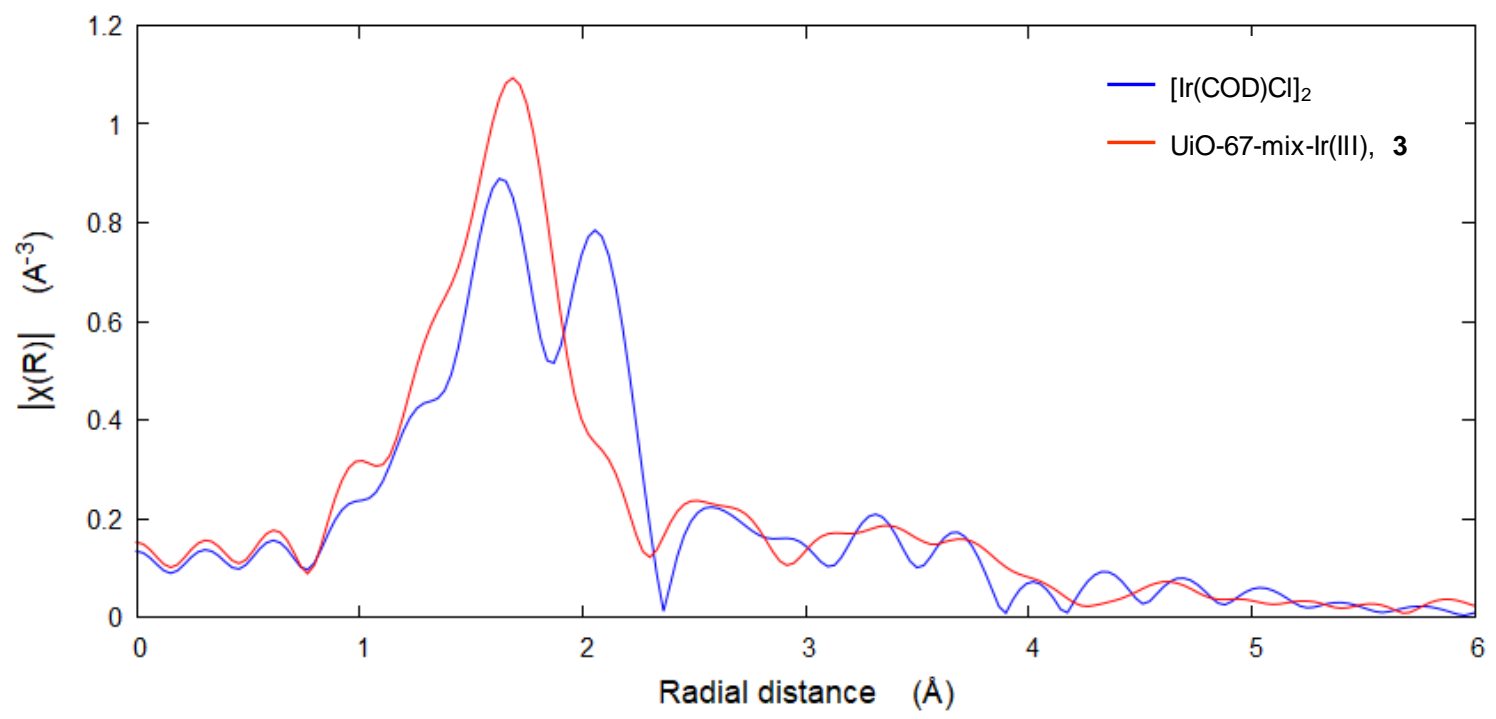

Figure S22. The magnitude part of the $k^{2}$-weighted EXAFS of UiO-67-mix-Ir(III), 3 and $[\operatorname{lr}(\mathrm{COD}) \mathrm{Cl}]_{2}$. 


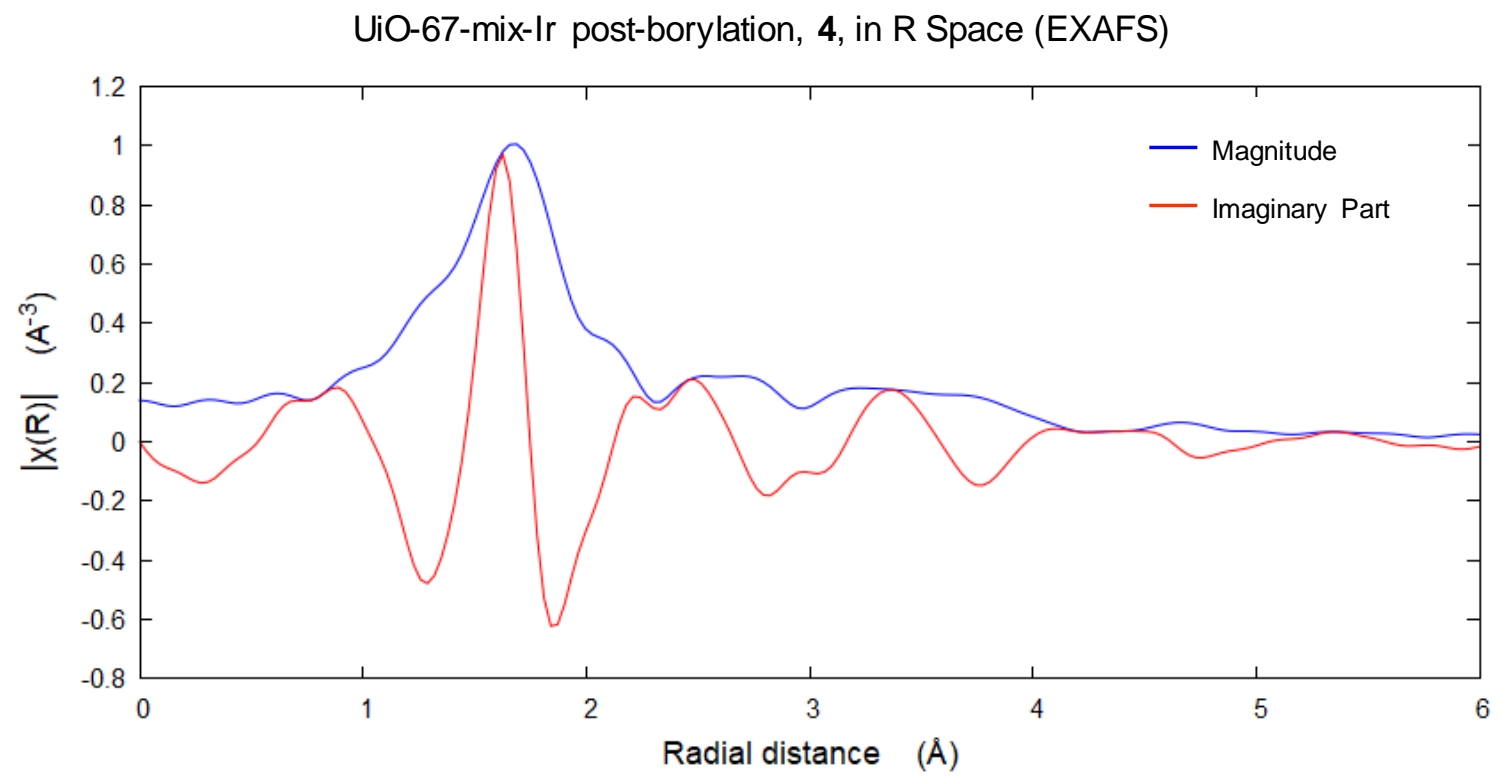

Figure S23. The magnitude and imaginary part of the $k^{2}$-weighted EXAFS of UiO-67-mix-Ir postborylation, 4.

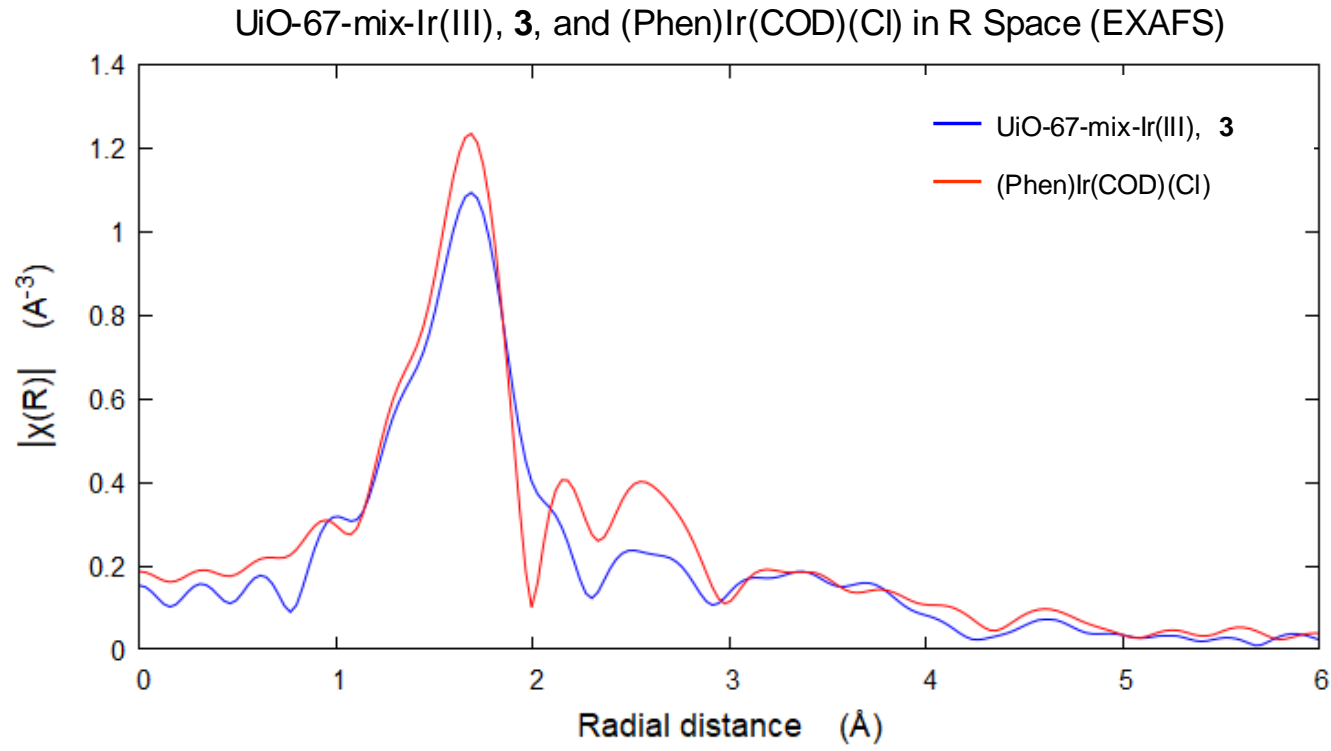

Figure S24. The magnitude part of the $\mathrm{k}^{2}$-weighted EXAFS of UiO-67-mix-Ir(III), 3 and (Phen) $\operatorname{Ir}(\mathrm{COD})(\mathrm{Cl})$. 
Table S8. Ir $L_{\text {III }}$ Edge EXAFS Fitting Parameters

\begin{tabular}{cccccc}
\hline Sample & $\begin{array}{c}\text { Scattering } \\
\text { Path* }\end{array}$ & $\begin{array}{c}\text { Coordination } \\
\text { Number }\end{array}$ & $\mathrm{R}(\AA)$ & $\begin{array}{c}\Delta \sigma^{2}\left(\times 10^{3}\right. \\
\left.\AA^{2}\right)\end{array}$ & $\mathrm{E}_{0}(\mathrm{eV})$ \\
\hline \multirow{2}{*}[\operatorname{lr}(\mathrm{COD})\mathrm{Cl}]{$_{2}$} & $\mathrm{Ir}-\mathrm{C}$ & 4 & $2.08 \pm 0.01$ & $2.9 \pm 0.5$ & $4.0 \pm 0.6$ \\
& $\mathrm{Ir}-\mathrm{Cl}$ & 2 & $2.39 \pm 0.01$ & $4.7 \pm 0.5$ & \\
& $\mathrm{Ir}-\mathrm{C} / \mathrm{N}$ & 6 & $2.10 \pm 0.01$ & $2.3 \pm 1.1$ & \\
$($ Phen) $\operatorname{lr}(\mathrm{COD})(\mathrm{Cl})$ & $\mathrm{Ir}-\mathrm{Cl}$ & 1 & $2.55 \pm 0.01$ & $4.0 \pm 4.2$ & $3.0 \pm 1.8$ \\
& $\mathrm{Ir}-\mathrm{C} / \mathrm{N} / \mathrm{O}$ & $8.6 \pm 0.9$ & $2.13 \pm 0.01$ & $8.1 \pm 1.7$ & $5.2 \pm 1.3$
\end{tabular}

"The amplitude reduction factor $\left(\mathrm{S}_{0}{ }^{2}\right)$ was determined to be 0.78 from $[\operatorname{lr}(\mathrm{COD}) \mathrm{Cl}]_{2}$ and was held constant at this value for all other fits.

\section{XPS data}

UiO-67-mix-Ir(I) (2)

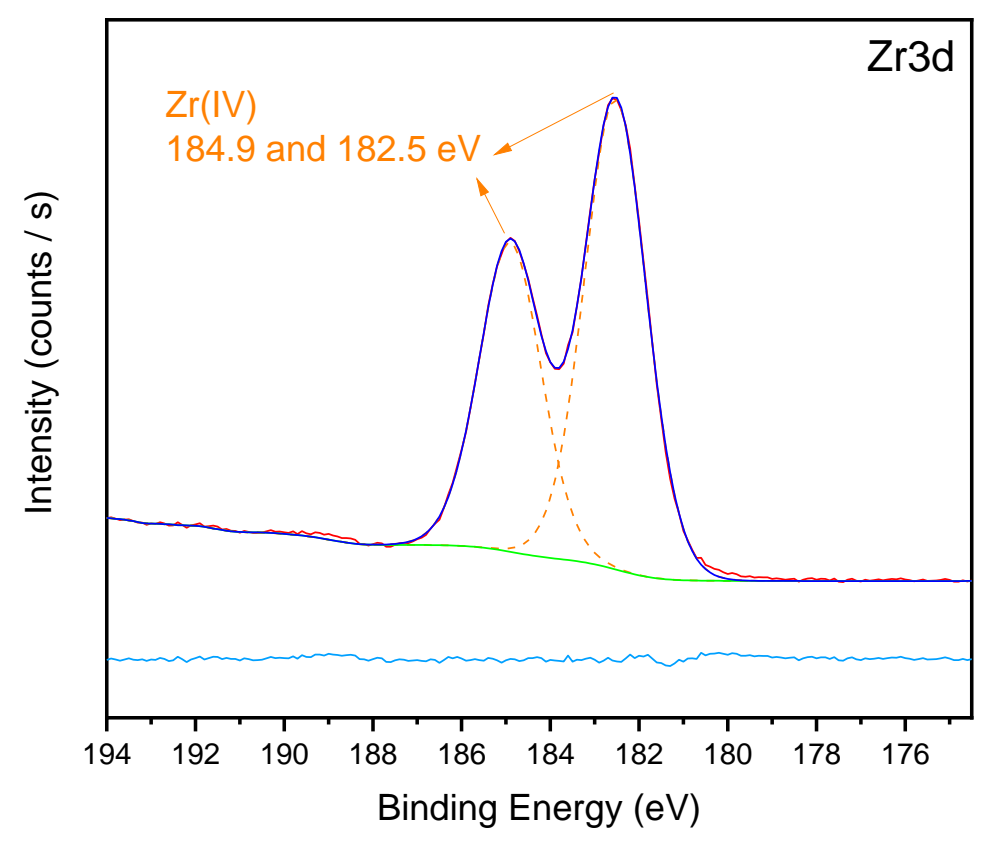

Figure S25. XPS spectra showing the binding energy of UiO-67-mix-Ir(I) at the $\mathrm{Zr}$ 3d region. Raw data (red), fitted peaks (orange dashed), fitting envelope (blue), background (green), residuals (light blue). 


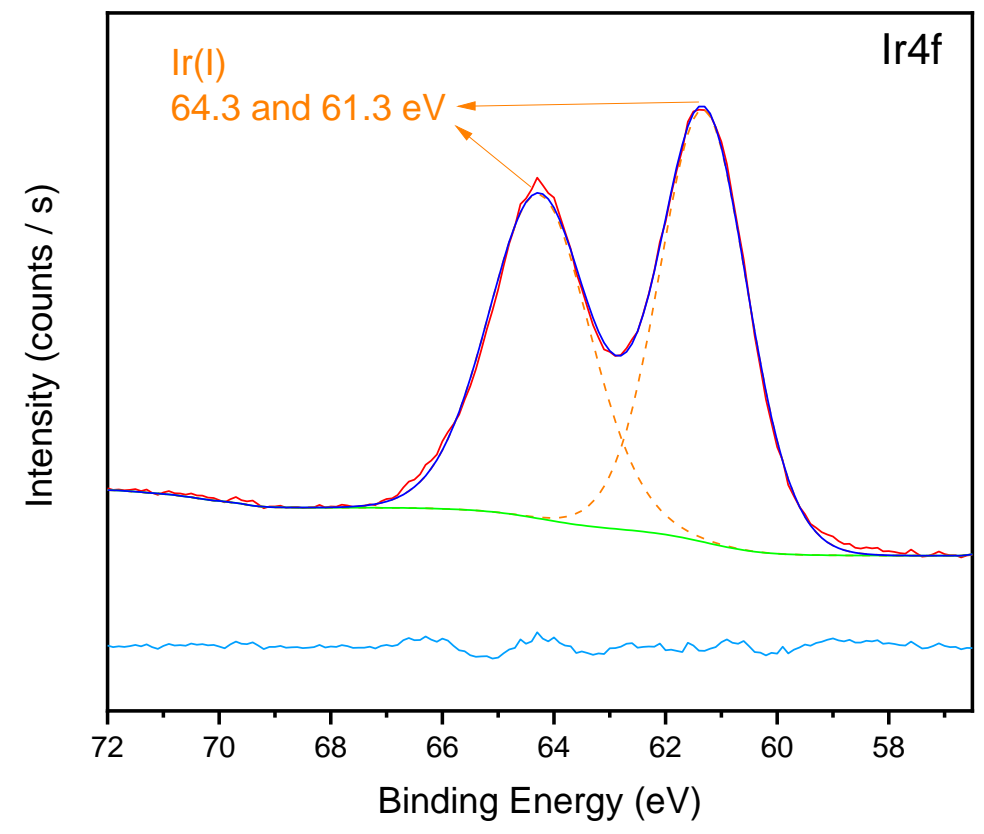

Figure S26. XPS spectra showing the binding energy of UiO-67-mix-Ir $(\mathrm{I})$ at the $\operatorname{Ir} 4 \mathrm{f}$ region. Raw data (red), fitted peaks (orange dashed), fitting envelope (blue), background (green), residuals (light blue).

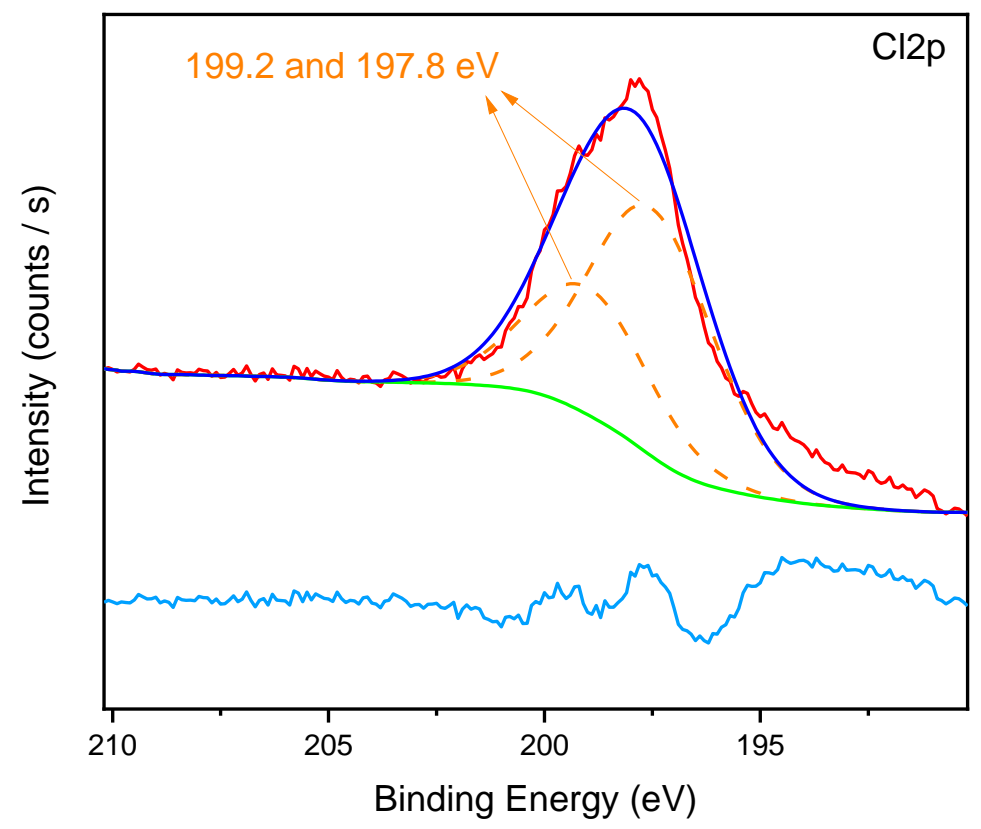

Figure S27. XPS spectra showing the binding energy of UiO-67-mix-Ir(I) at the $\mathrm{Cl} 2 \mathrm{p}$ region. Raw data (red), fitted peaks (orange dashed), fitting envelope (blue), background (green), residuals (light blue). 


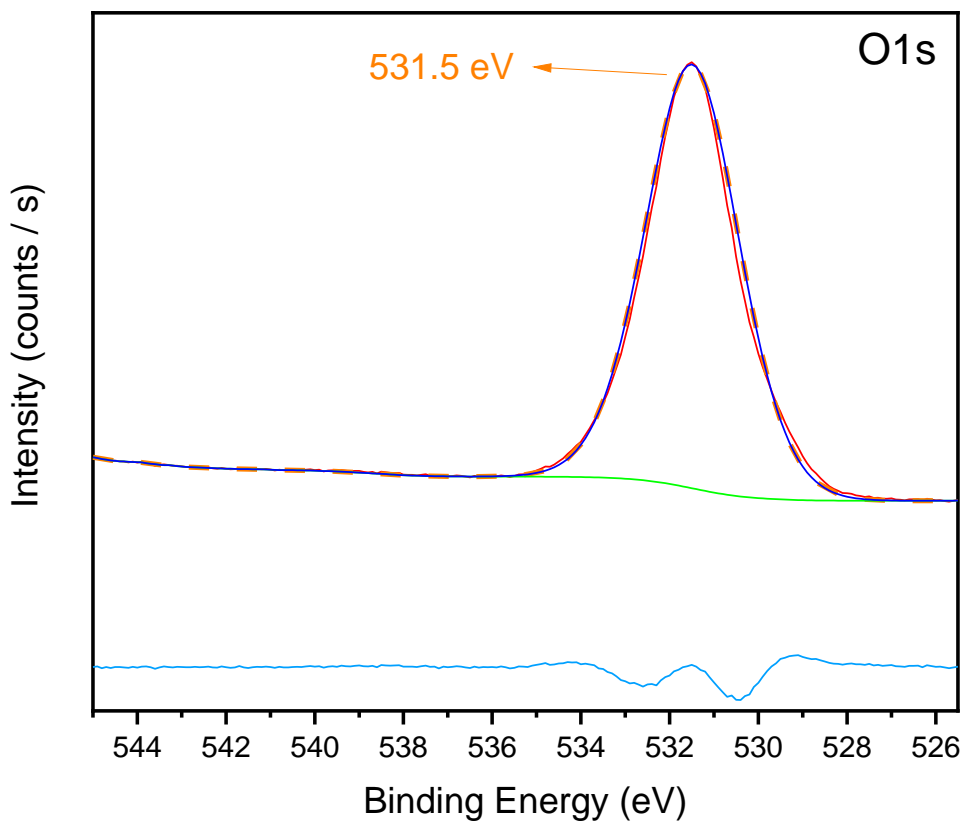

Figure S28. XPS spectra showing the binding energy of UiO-67-mix-Ir(I) at the $O 1$ s region. Raw data (red), fitted peaks (orange dashed), fitting envelope (blue), background (green), residuals (light blue).

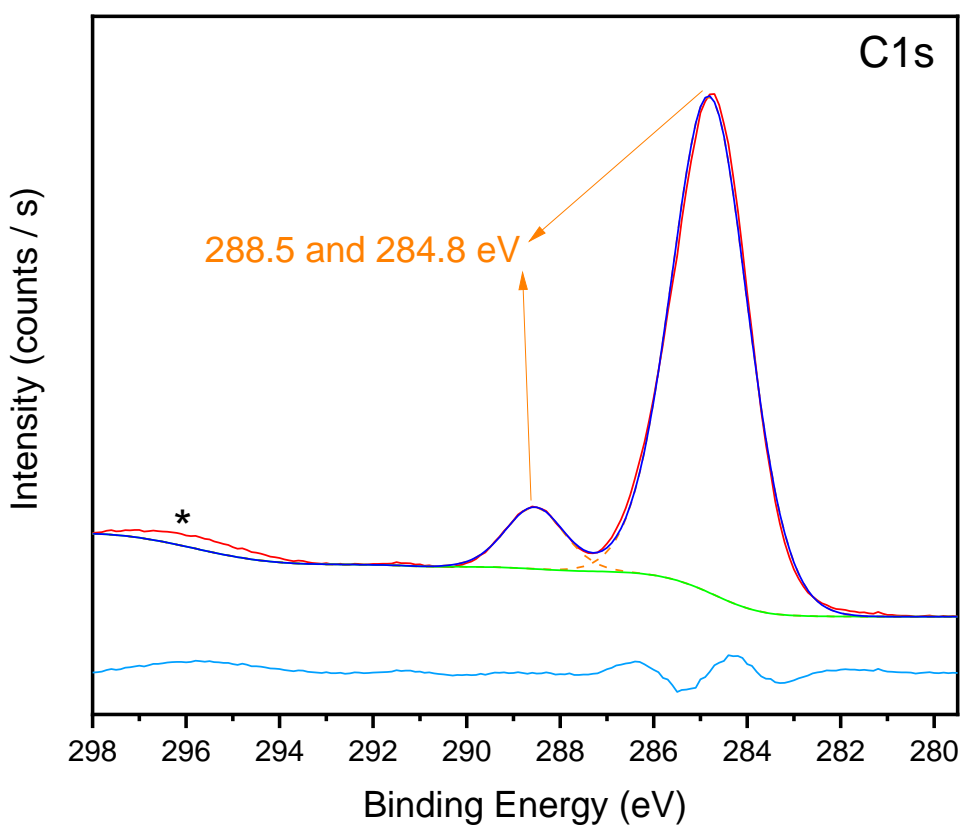

Figure S29. XPS spectra showing the binding energy of UiO-67-mix-Ir(l) at the $C 1$ s region. Raw data (red), fitted peaks (orange dashed), fitting envelope (blue), background (green), residuals (light blue). *Overlapping Ir 4d band 
UiO-67-mix-Ir(III) (3)

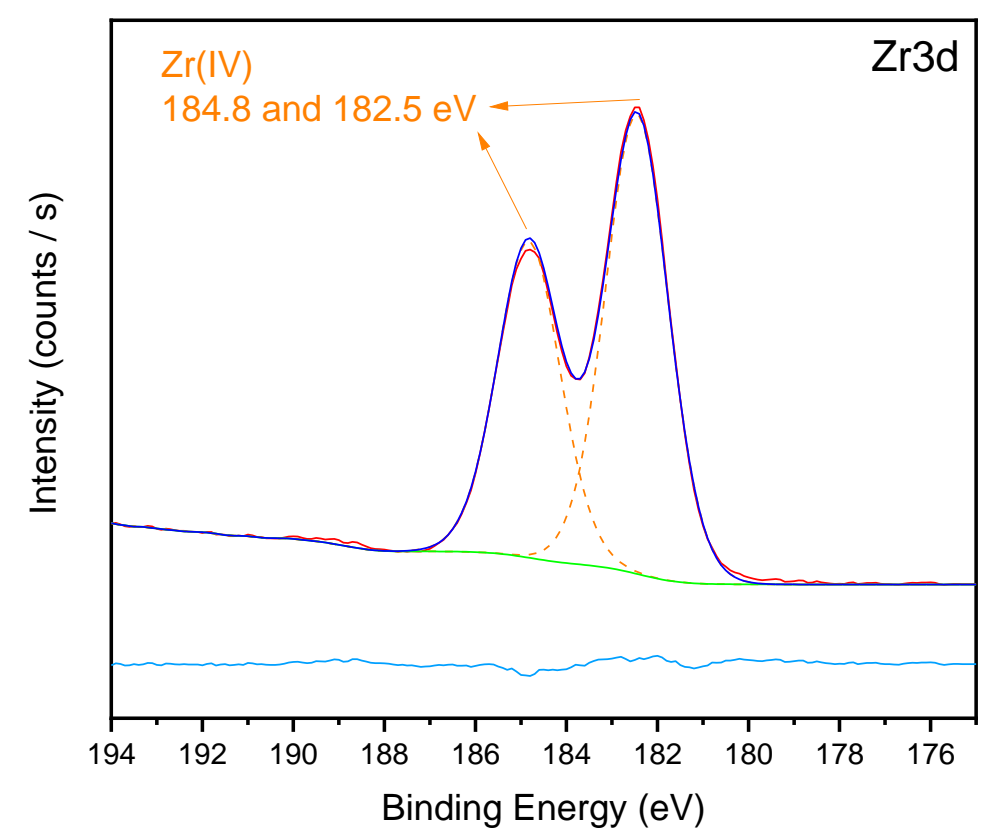

Figure S30. XPS spectra showing the binding energy of UiO-67-mix-Ir(III) at the Zr 3d region. Raw data (red), fitted peaks (orange dashed), fitting envelope (blue), background (green), residuals (light blue).

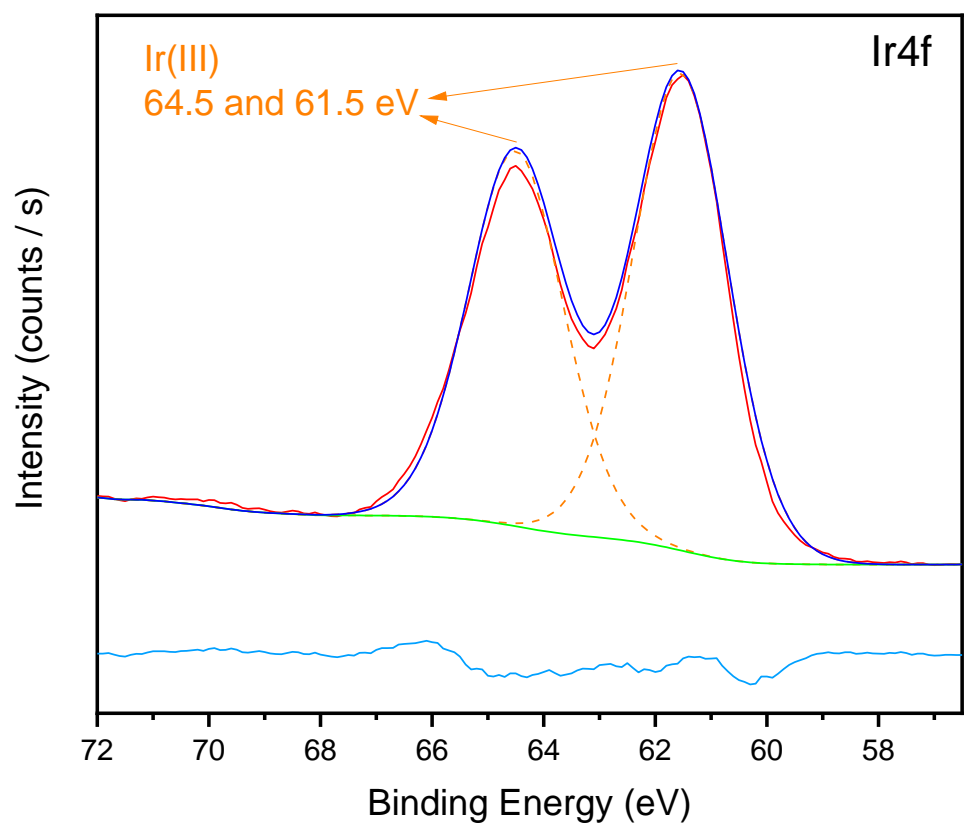

Figure S31. XPS spectra showing the binding energy of UiO-67-mix-Ir(III) at the Ir $4 \mathrm{f}$ region. Raw data (red), fitted peaks (orange dashed), fitting envelope (blue), background (green), residuals (light blue). 


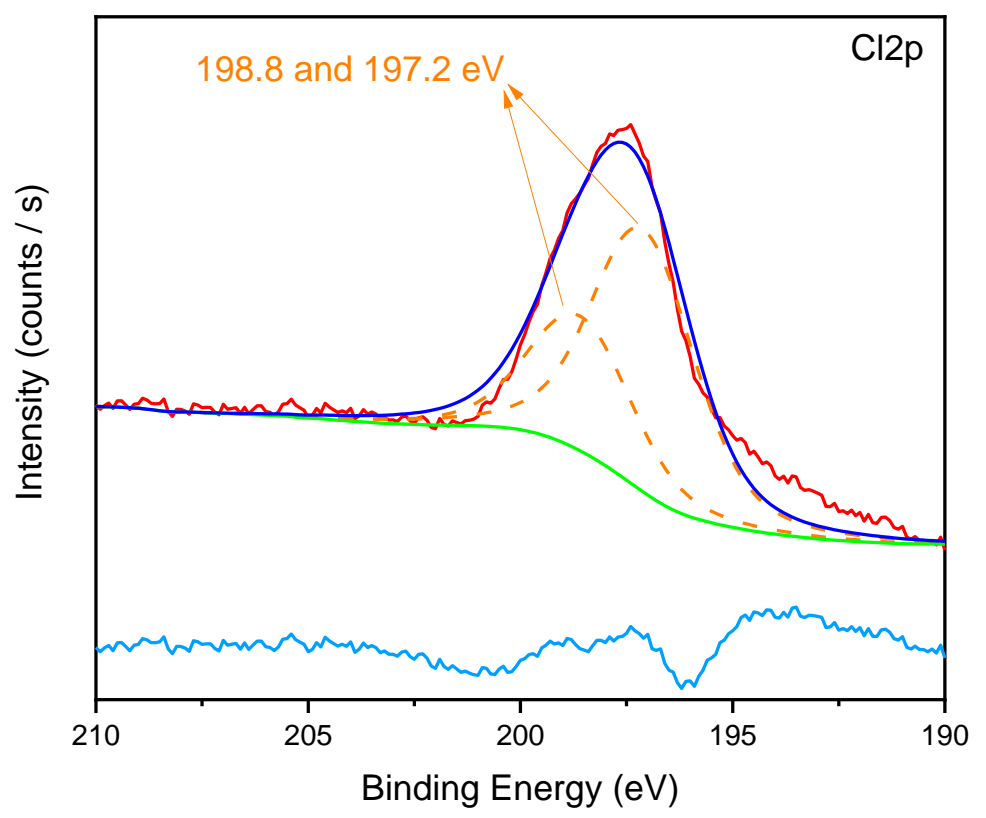

Figure S32. XPS spectra showing the binding energy of UiO-67-mix-Ir(III) at the $\mathrm{Cl} 2 \mathrm{p}$ region. Raw data (red), fitted peaks (orange dashed), fitting envelope (blue), background (green), residuals (light blue).

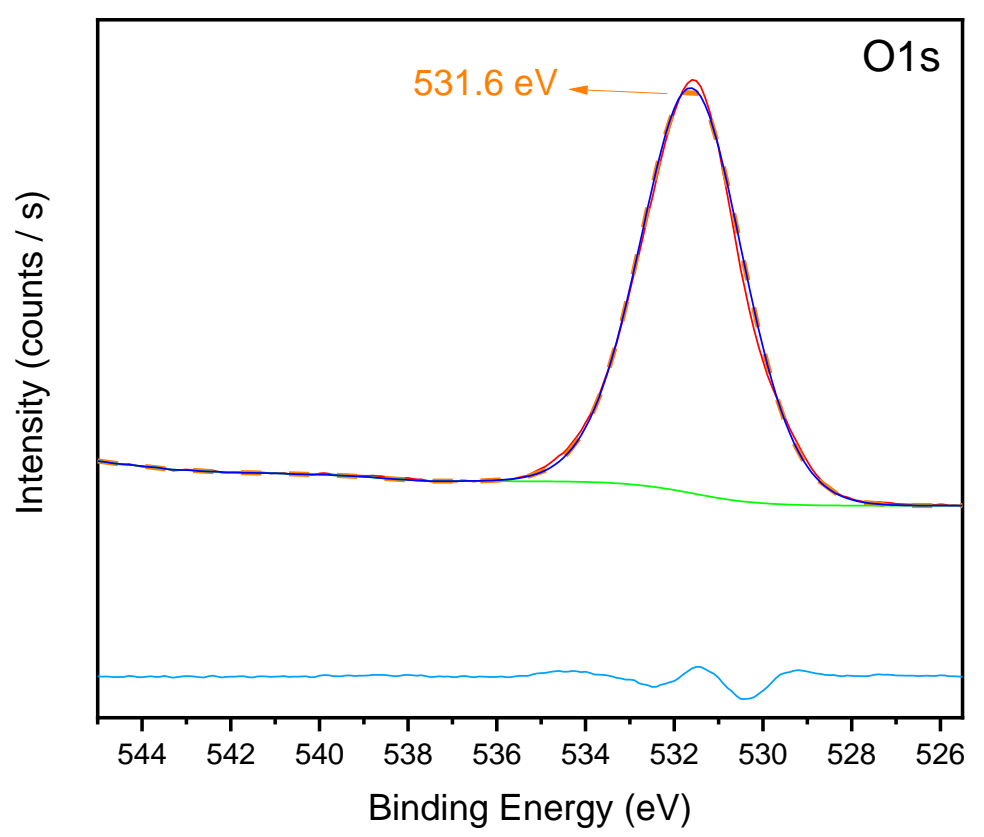

Figure S33. XPS spectra showing the binding energy of UiO-67-mix-Ir(III) at the $\mathrm{O} 1 \mathrm{~s}$ region. Raw data (red), fitted peaks (orange dashed), fitting envelope (blue), background (green), residuals (light blue). 
UiO-67-mix-Ir post-borylation (4)

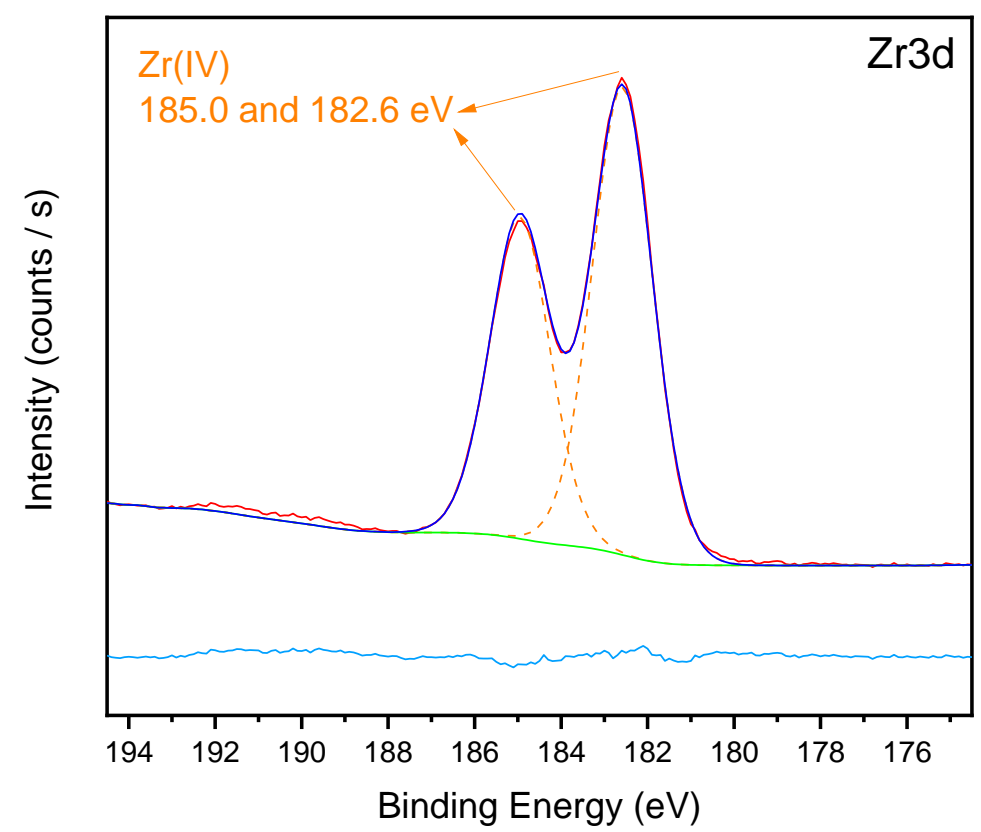

Figure S34. XPS spectra showing the binding energy of UiO-67-mix-Ir post-borylation at the Zr $3 d$ region. Raw data (red), fitted peaks (orange dashed), fitting envelope (blue), background (green), residuals (light blue).

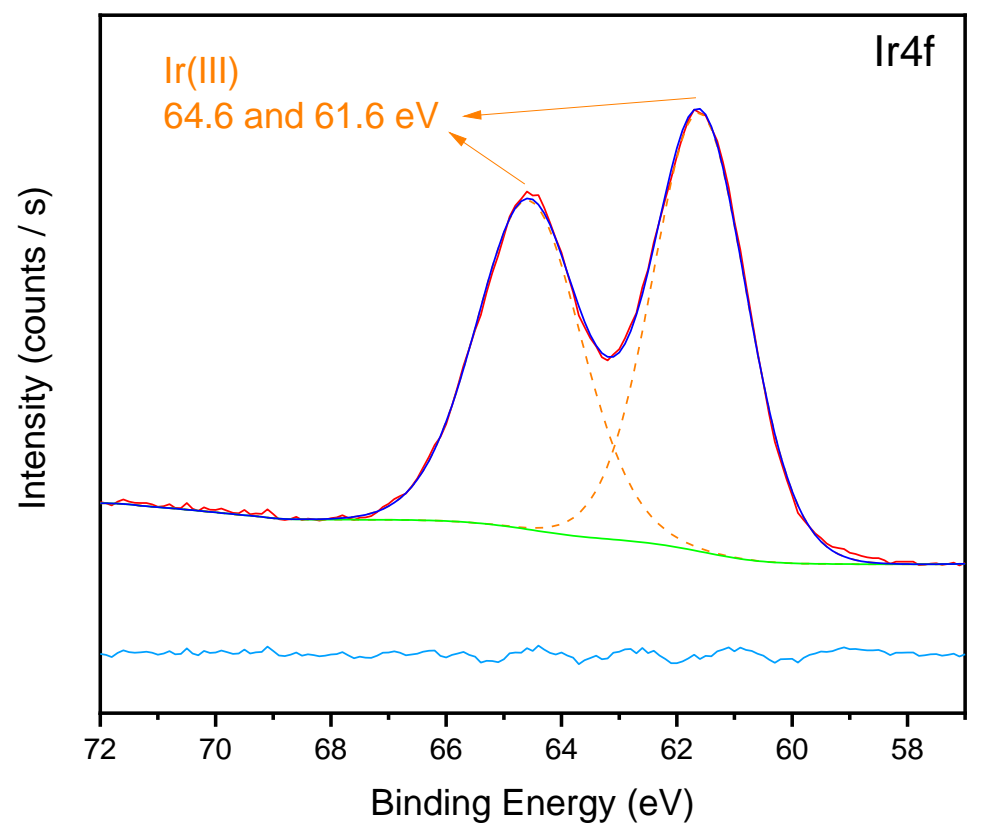

Figure S35. XPS spectra showing the binding energy of UiO-67-mix-Ir post-borylation at the Ir $4 \mathrm{f}$ region. Raw data (red), fitted peaks (orange dashed), fitting envelope (blue), background (green), residuals (light blue). 


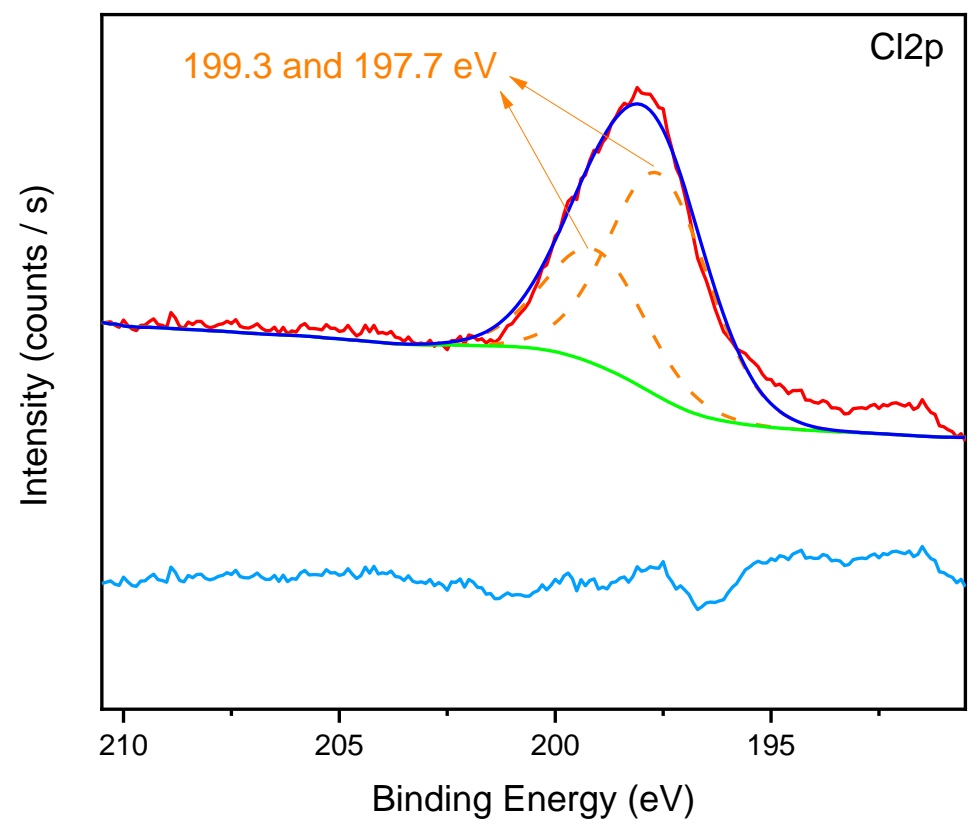

Figure S36. XPS spectra showing the binding energy of UiO-67-mix-Ir post-borylation at the $\mathrm{Cl}$ $2 p$ region. Raw data (red), fitted peaks (orange dashed), fitting envelope (blue), background (green), residuals (light blue).

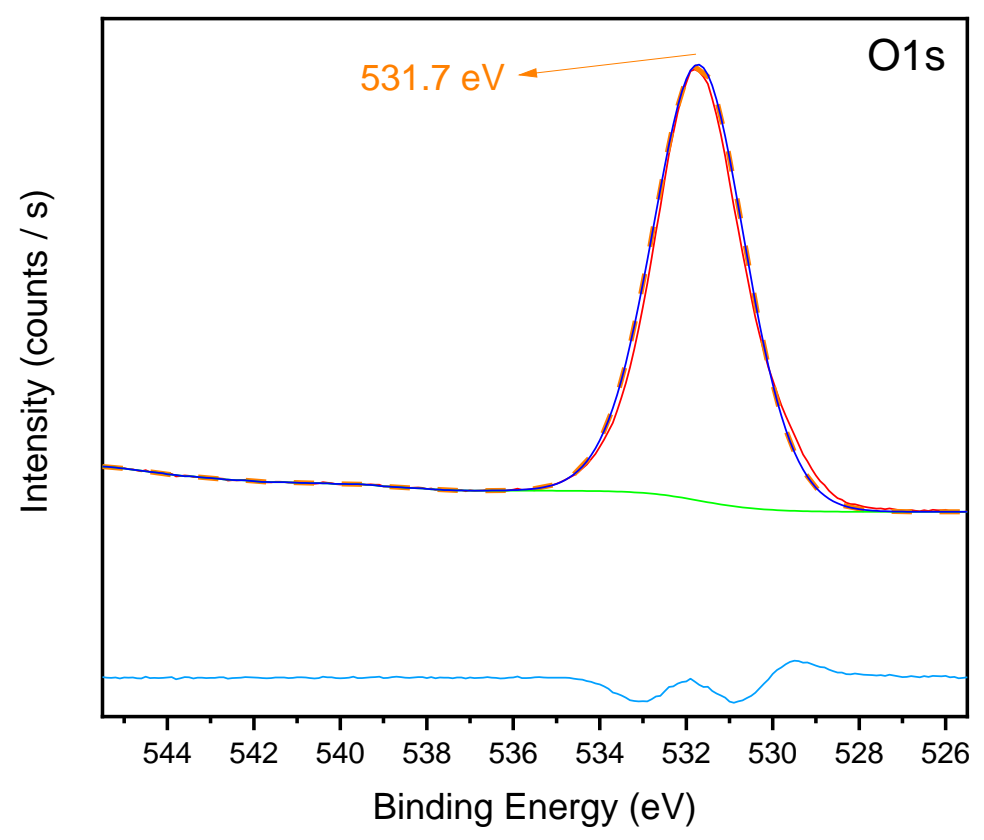

Figure S37. XPS spectra showing the binding energy of UiO-67-mix-Ir post-borylation at the $O$ 1s region. Raw data (red), fitted peaks (orange dashed), fitting envelope (blue), background (green), residuals (light blue). 


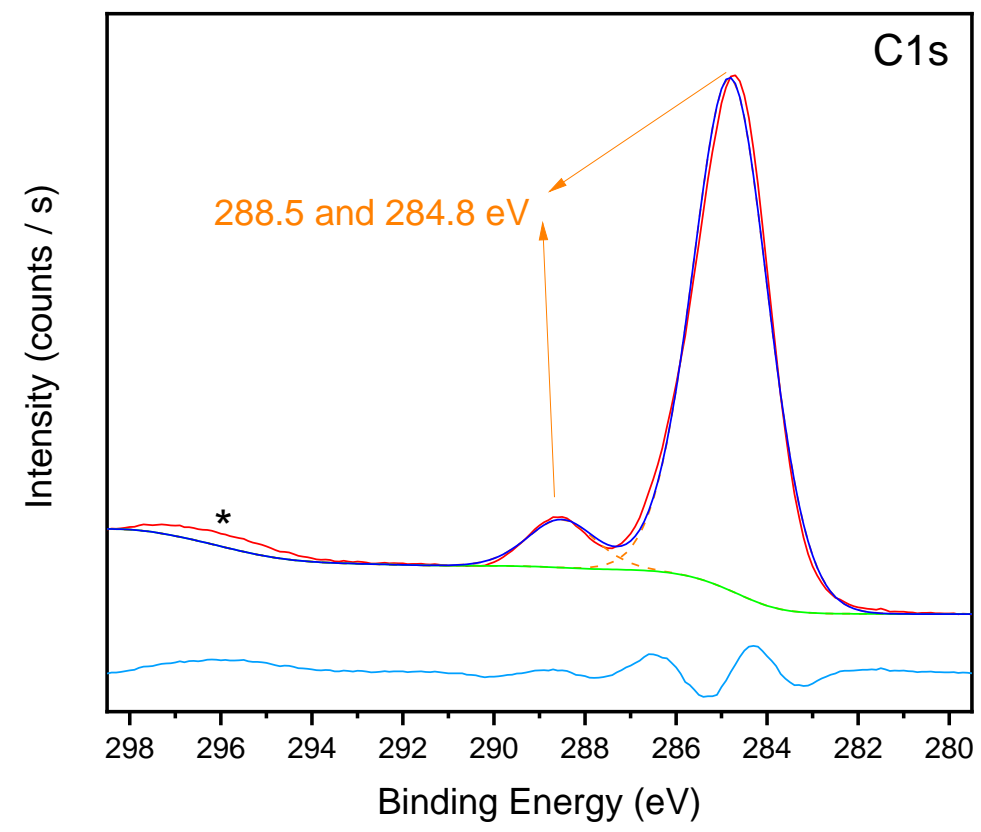

Figure S38. XPS spectra showing the binding energy of UiO-67-mix-Ir(III)-post-boryl at the C 1s region. Raw data (red), fitted peaks (orange dashed), fitting envelope (blue), background (green), residuals (light blue). *Overlapping Ir $4 \mathrm{~d}$ band

\section{PDF data}

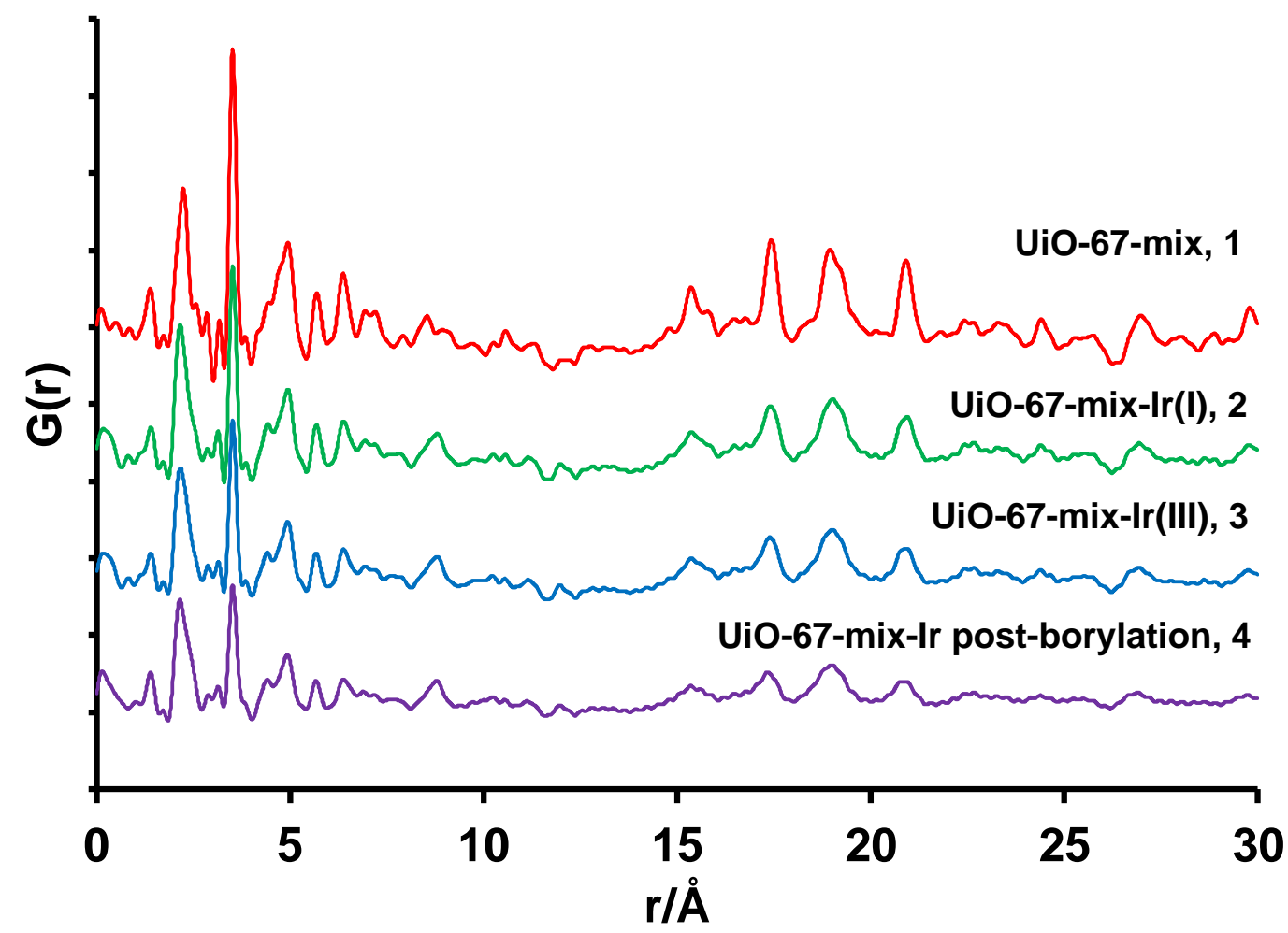

Figure S39. PDF analysis of x-ray diffraction patterns for UiO-67-mix and Ir-metalated samples. 
dPDF data

Table S9. dPDF data for UiO-67-mix-Ir(I), 2

\begin{tabular}{ccccc}
\hline $\begin{array}{c}\text { Assigned Atom } \\
\text { Pairs }\end{array}$ & Center & Height & Area & $\begin{array}{c}\text { Full Width at } \\
\text { Half Maximum } \\
\text { (FWHM) }\end{array}$ \\
\hline Ir-C/N/O & 2.11 & 1.31 & 0.224 & 0.161 \\
$\quad \mathrm{Ir}-\mathrm{Cl}$ & 2.46 & 0.547 & 0.0856 & 0.147 \\
$\begin{array}{c}\mathrm{Ir} . . \mathrm{C} \text { (on COD } \\
\text { and/or ligand) }\end{array}$ & 3.05 & 1.11 & 0.218 & 0.184 \\
\hline
\end{tabular}

Table S10. dPDF data for UiO-67-mix-Ir(III), 3

\begin{tabular}{ccccc}
\hline $\begin{array}{c}\text { Assigned Atom } \\
\text { Pairs }\end{array}$ & Center & Height & Area & $\begin{array}{c}\text { Full Width at } \\
\text { Half Maximum } \\
\text { (FWHM) }\end{array}$ \\
\hline $\mathrm{Ir}-\mathrm{C} / \mathrm{N} / \mathrm{O}$ & 2.11 & 1.20 & 0.214 & 0.168 \\
$\mathrm{Ir}-\mathrm{Cl}$ & 2.44 & 0.629 & 0.105 & 0.157 \\
$\begin{array}{c}\mathrm{Ir} \ldots \mathrm{C} \text { (on COD } \\
\text { and/or ligand) }\end{array}$ & 3.03 & 1.01 & 0.213 & 0.198 \\
\hline
\end{tabular}

Table S11. dPDF data for UiO-67-mix-Ir post-borylation, 4

\begin{tabular}{ccccc}
\hline $\begin{array}{c}\text { Assigned Atom } \\
\text { Pairs }\end{array}$ & Center & Height & Area & $\begin{array}{c}\text { Full Width at } \\
\text { Half Maximum } \\
\text { (FWHM) }\end{array}$ \\
\hline $\begin{array}{c}\mathrm{Ir}-\mathrm{C} / \mathrm{N} / \mathrm{O} \\
\mathrm{Ir}-\mathrm{Cl}\end{array}$ & 2.11 & 1.46 & 0.262 & 0.168 \\
$\begin{array}{c}\mathrm{Ir} . . \mathrm{C} \text { (on COD } \\
\text { and/or ligand) }\end{array}$ & 2.44 & 0.968 & 0.162 & 0.158 \\
\hline
\end{tabular}




\section{DED data}

UiO-67-mix-Ir(I) (2)

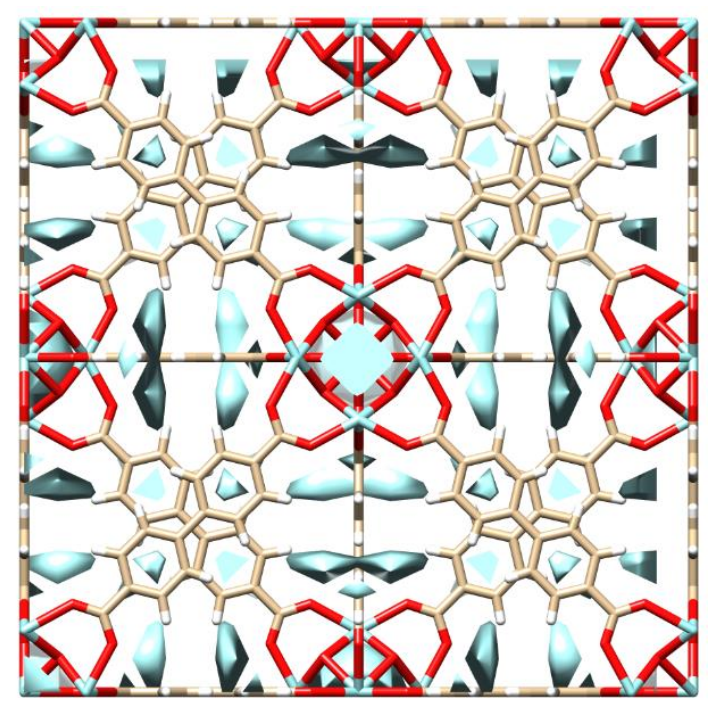

Figure S40. DED analysis of UiO-67-mix-Ir(I), full view, solid bulbs represent electron density. Varying electron density at the $\mathrm{Zr}$ node may be due to the presence of defects following solvothermal deposition or residual iridium grafted on the node.

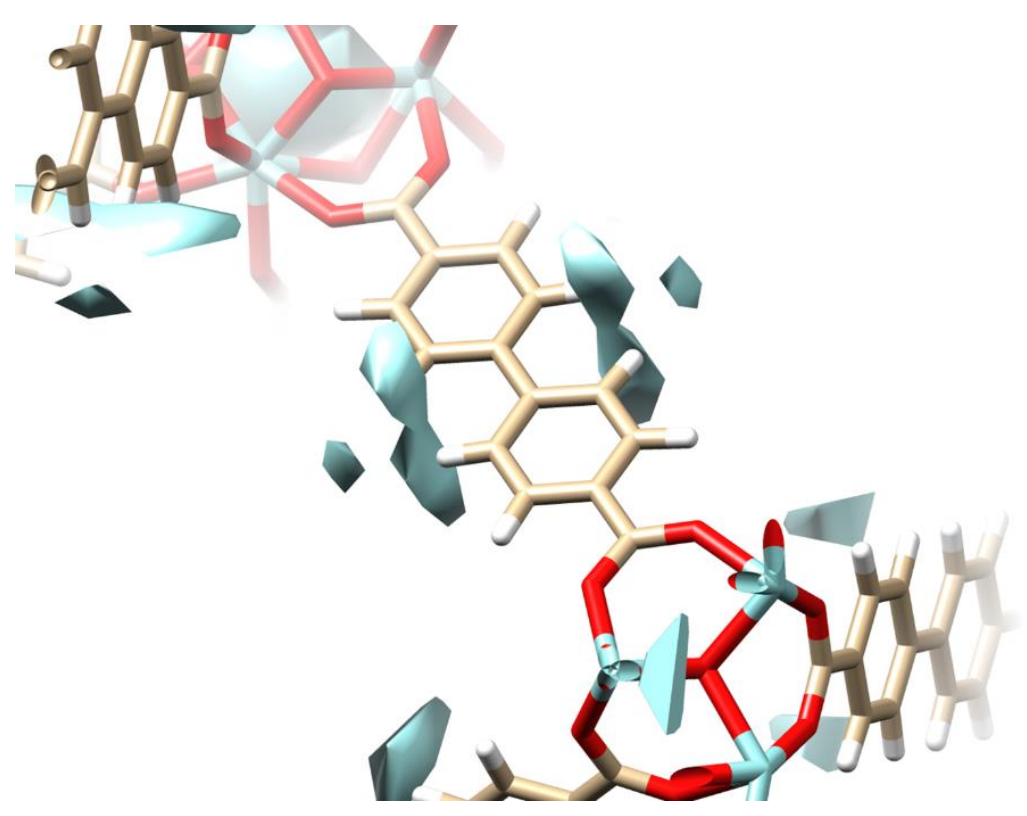

Figure S41. DED analysis of UiO-67-mix-Ir(I), zoomed-in ligand view, solid bulbs represent electron density. Electron density is centered around functionalized linker, likely represented by BPDC based on a pure model of UiO-67. 
UiO-67-mix-Ir(III) (3)

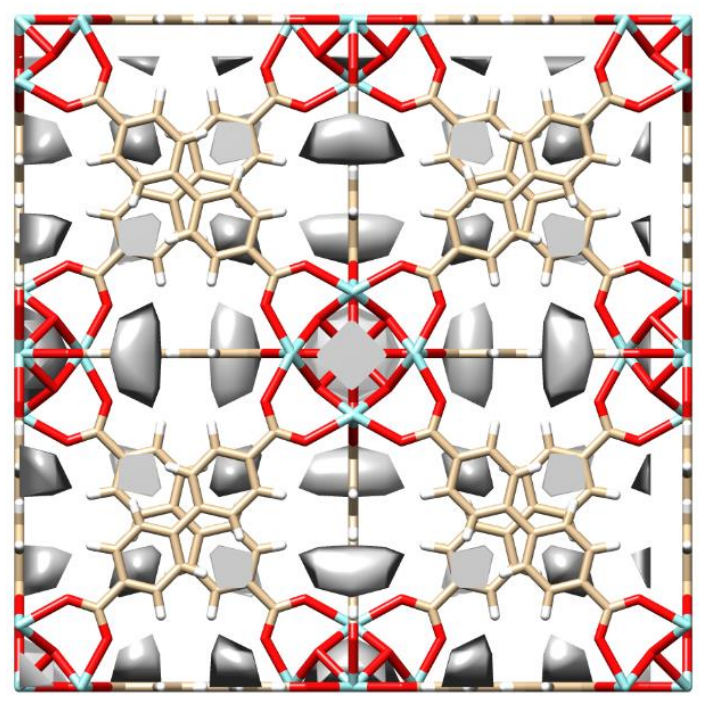

Figure S42. DED analysis of UiO-67-mix-Ir(III), full view, solid bulbs represent electron density. Varying electron density at the $\mathrm{Zr}$ node may be due to the presence of defects following solvothermal deposition or residual iridium grafted on the node.

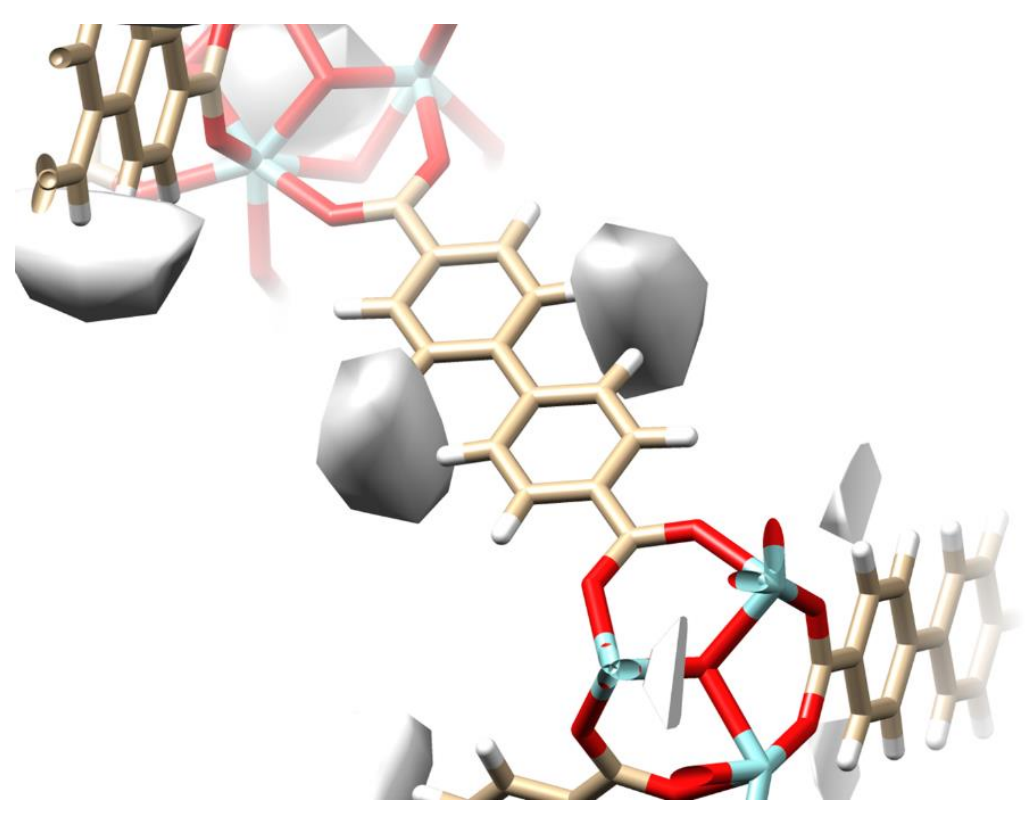

Figure S43. DED analysis of UiO-67-mix-Ir(III), zoomed-in ligand view, solid bulbs represent electron density. Electron density is centered around functionalized linker, likely represented by BPDC based on a pure model of UiO-67. 


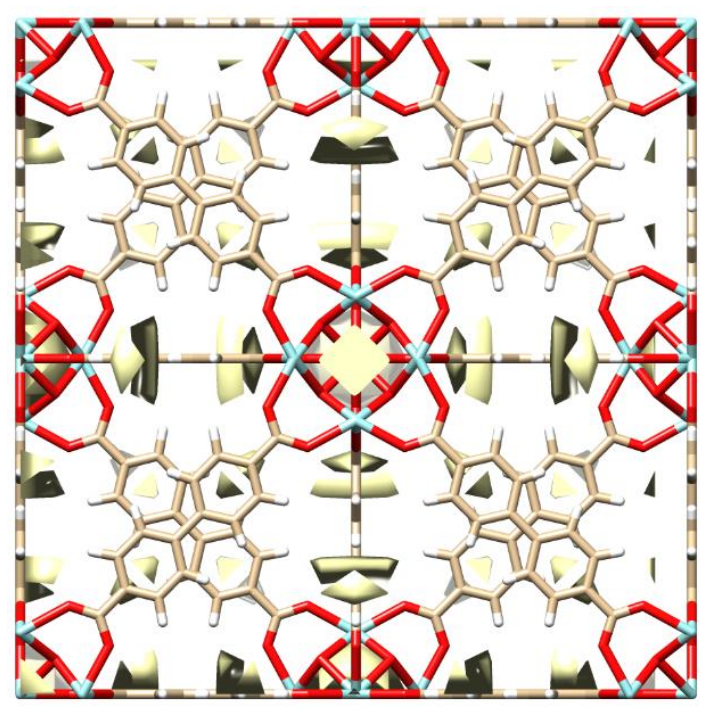

Figure S44. DED analysis of UiO-67-mix-Ir post-borylation, full view, solid bulbs represent electron density. Varying electron density at the $\mathrm{Zr}$ node may be due to the presence of defects following solvothermal deposition or residual iridium grafted on the node.

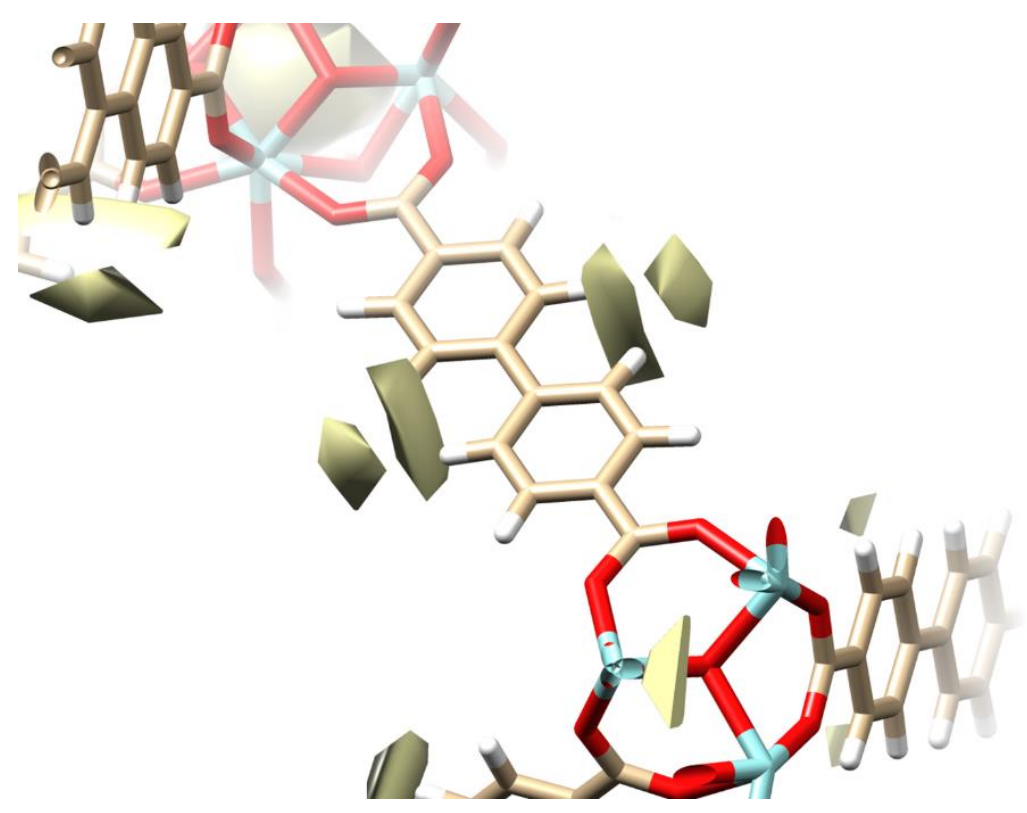

Figure S45. DED analysis of UiO-67-mix-Ir post-borylation, zoomed-in ligand view, solid bulbs represent electron density. Electron density is centered around functionalized linker, likely represented by BPDC based on a pure model of UiO-67. 


\section{Benzene Sorption Data}

\section{UiO-67-mix (1)}

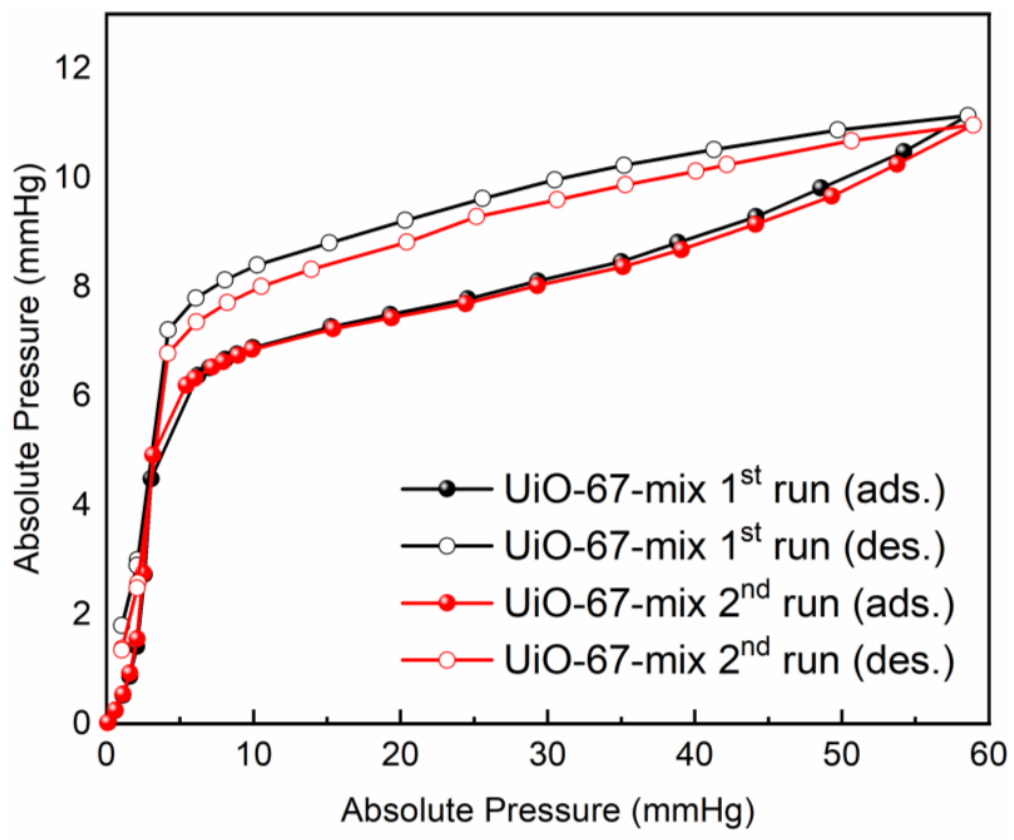

Figure S46. Vapor-phase benzene sorption ( $1^{\text {st }}$ and $2^{\text {nd }}$ cycle) with UiO-67-mix, adsorption and desorption shown.

\section{UiO-67-mix-Ir(III) (3)}

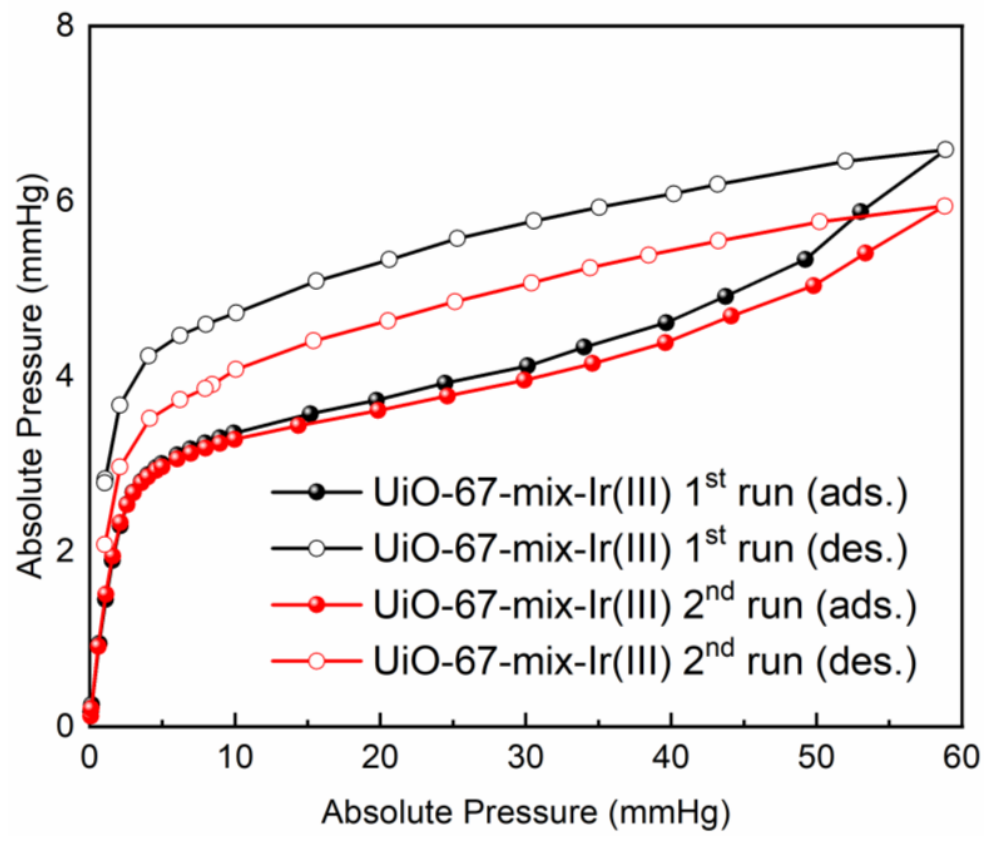

Figure S47. Vapor-phase benzene sorption ( $1^{\text {st }}$ and $2^{\text {nd }}$ cycle) with UiO-67-mix-Ir(III), adsorption and desorption shown. 


\section{Catalysis}
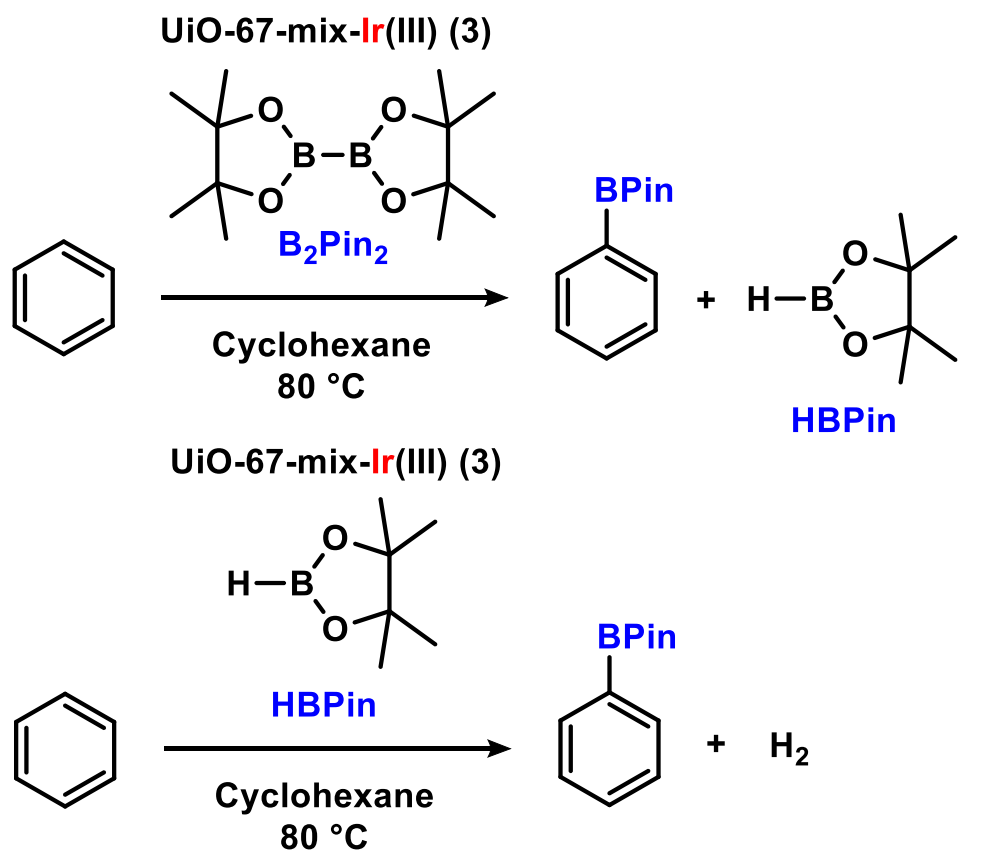

Figure S48. Borylation of benzene forming an equivalent of PhBPin and HBPin. The in situgenerated HBPin can then be used for borylation of another equivalent of benzene to form PhBPin and $\mathrm{H}_{2}$.

\section{General procedure for kinetic monitoring of benzene borylation reaction with $\mathrm{B}_{2} \mathrm{Pin}_{2}$ and HBPin}

In a glovebox under inert atmosphere, to an oven-dried 2-5 $\mathrm{mL}$ Biotage microwave vial charged with stir bar was added 3, anhydrous cyclohexane, anhydrous benzene, HBPin, anhydrous hexadecane as the internal standard, and $\mathrm{B}_{2} \mathrm{Pin}_{2}$ as a stock solution in anhydrous cyclohexane. The microwave vial was then sealed with a cap that was crimped. It was stirred at $1000 \mathrm{rpm}$ using an aluminum block in the center of a Heidolph hotplate with magnetic stirring at room temperature and an initial aliquot was taken via needle and syringe using an outlet purge needle to relieve any pressure. The aliquot was diluted with $\sim 0.2 \mathrm{~mL}$ of anhydrous cyclohexane and solids were filtered using a GE Healthcare Whatman ${ }^{\mathrm{TM}}$ Mini-UniPrep ${ }^{\mathrm{TM}}$ Syringeless Filter vial. The microwave vial was then removed from the aluminum block and the temperature of the hotplate was set to $80^{\circ} \mathrm{C}$. The reaction was then placed on the heated aluminum block and the liquid meniscus was further wrapped in aluminum foil for insulation. The reaction was monitored over time by GC-FID. Note, HBPin was difficult to quantify by GC-FID thus the appearance of PhBPin was used as confirmation that HBPin was a viable boron source for this reaction.

Table S12. Conditions for Kinetic Monitoring of Benzene Borylation with HBPin

\begin{tabular}{ccccccccc}
\hline Entry & $\begin{array}{c}\text { Catalyst } \\
(\mathbf{m g}, \\
\text { targeted) }\end{array}$ & $\begin{array}{c}\text { Catalyst } \\
(\mathbf{m g}, \\
\text { measured) }\end{array}$ & $\begin{array}{c}\text { Benzene } \\
(\mathbf{M})\end{array}$ & $\begin{array}{c}\mathrm{B}_{2} \text { Pin2 } \\
(\mathbf{M}, \\
\text { targeted) }\end{array}$ & $\begin{array}{c}\mathrm{B}_{2} \text { Pin2 } \\
(\mathbf{M}, \\
\text { measure } \\
\mathbf{d} \text { by GC- } \\
\text { FID) }\end{array}$ & $\begin{array}{c}\text { HBPin } \\
(\mathbf{M}, \\
\text { targeted })\end{array}$ & $\begin{array}{c}\text { Hexadecane } \\
(\mathbf{M})\end{array}$ & $\begin{array}{c}\text { Total } \\
\text { Vol } \\
(\mathbf{m L})\end{array}$ \\
\hline 1 & 40 & 40.9 & 0.48 & 0.073 & 0.074 & 0.023 & 0.023 & 3.02 \\
\hline
\end{tabular}



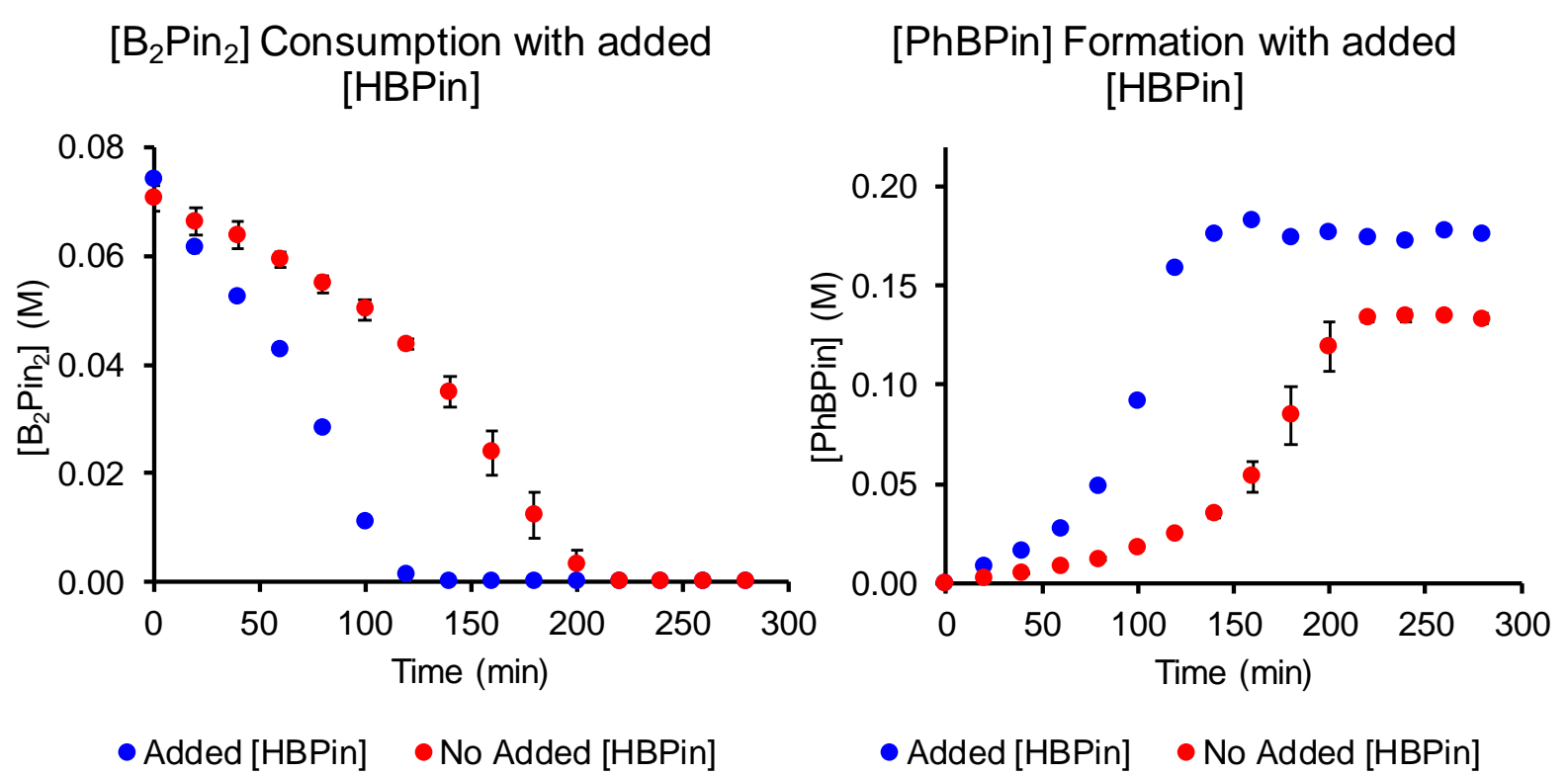

Figure S49. Effect of added [HBPin] on consumption of $\left[\mathrm{B}_{2} \mathrm{Pin}_{2}\right]$ and formation of [PhBPin] overlaid with kinetic plots from Table S19 entries 1-3. Error bars on red plot represent standard deviation (sample) of triplicate runs.

\section{General procedure for heterogeneous benzene borylation reaction with HBPin as the boron source}

In a glovebox under inert atmosphere, to an oven-dried 2-5 $\mathrm{mL}$ Biotage microwave vial charged with stir bar was added UiO-67-mix-Ir(III) $(3,13 \mathrm{mg})$, anhydrous cyclohexane $(0.79 \mathrm{~mL})$, anhydrous benzene $(0.17 \mathrm{~mL}, 1.9 \mathrm{mmol})$, HBPin $(21 \mu \mathrm{L}, 0.145 \mathrm{mmol})$, and anhydrous hexadecane $(20 \mu \mathrm{L}, 0.068 \mathrm{mmol})$ as the internal standard. The microwave vial was then sealed with a cap that was crimped. It was stirred at $80{ }^{\circ} \mathrm{C}$ at $500 \mathrm{rpm}$ for 3 hours using an aluminum block in the center of a Heidolph hotplate with magnetic stirring. The liquid meniscus was further wrapped in aluminum foil for insulation. After 3 hours, an aliquot was then taken with a needle and syringe as well as an outlet purge needle to relieve pressure. It was diluted with $\sim 0.2 \mathrm{~mL}$ of anhydrous cyclohexane and solids were filtered using a GE Healthcare Whatman ${ }^{\mathrm{TM}}$ MiniUniPrep $^{\text {TM }}$ Syringeless Filter vial, after which it was analyzed by GC-FID. Note, HBPin was difficult to quantify by GC-FID thus the appearance of PhBPin was used as confirmation that HBPin was a viable boron source for this reaction.

Table S13. Formation of PhBPin with HBPin as the Boron Source

\section{Entry}

[PhBPin] (M) after $3 \mathrm{~h}$

1

0.198

\section{General procedure for kinetic monitoring of benzene borylation reaction with 2}

In a glovebox under inert atmosphere, to an oven-dried 2-5 mL Biotage microwave vial charged with stir bar was added 2, anhydrous cyclohexane, anhydrous benzene, anhydrous hexadecane 
as the internal standard, $\mathrm{B}_{2} \mathrm{Pin}_{2}$ as a stock solution in anhydrous cyclohexane. The microwave vial was then sealed with a cap that was crimped. It was stirred at $1000 \mathrm{rpm}$ using an aluminum block in the center of a Heidolph hotplate with magnetic stirring at room temperature and an initial aliquot was taken via needle and syringe using an outlet purge needle to relieve any pressure. The aliquot was diluted with $\sim 0.2 \mathrm{~mL}$ of anhydrous cyclohexane and solids were filtered using a GE Healthcare Whatman ${ }^{\mathrm{TM}}$ Mini-UniPrep ${ }^{\mathrm{TM}}$ Syringeless Filter vial. The microwave vial was then removed from the aluminum block and the temperature of the hotplate was set to $80{ }^{\circ} \mathrm{C}$. The reaction was then placed on the heated aluminum block and the liquid meniscus was further wrapped in aluminum foil for insulation. The reaction was monitored over time by GC-FID.

Table S14. Conditions for Kinetic Monitoring of Benzene Borylation with 2

\begin{tabular}{cccccccc}
\hline Entry & $\begin{array}{c}\text { Catalyst }(\mathbf{m g}, \\
\text { targeted) }\end{array}$ & $\begin{array}{c}\text { Catalyst }(\mathbf{m g}, \\
\text { measured })\end{array}$ & $\begin{array}{c}\text { Benzene } \\
(\mathbf{M})\end{array}$ & $\begin{array}{c}\mathbf{B}_{2} \text { Pin }_{2} \\
(\mathbf{M}, \\
\text { targeted })\end{array}$ & $\begin{array}{c}\mathbf{B}_{2} \text { Pin } \\
\text { measured } \\
\text { by GC-FID) }\end{array}$ & $\begin{array}{c}\text { Hexadecane } \\
(\mathbf{M})\end{array}$ & $\begin{array}{c}\text { Total } \\
\text { Volume } \\
(\mathbf{m L})\end{array}$ \\
\hline 1 & 40 & 40.9 & 1.93 & 0.073 & 0.071 & 0.023 & 3.02 \\
\hline
\end{tabular}
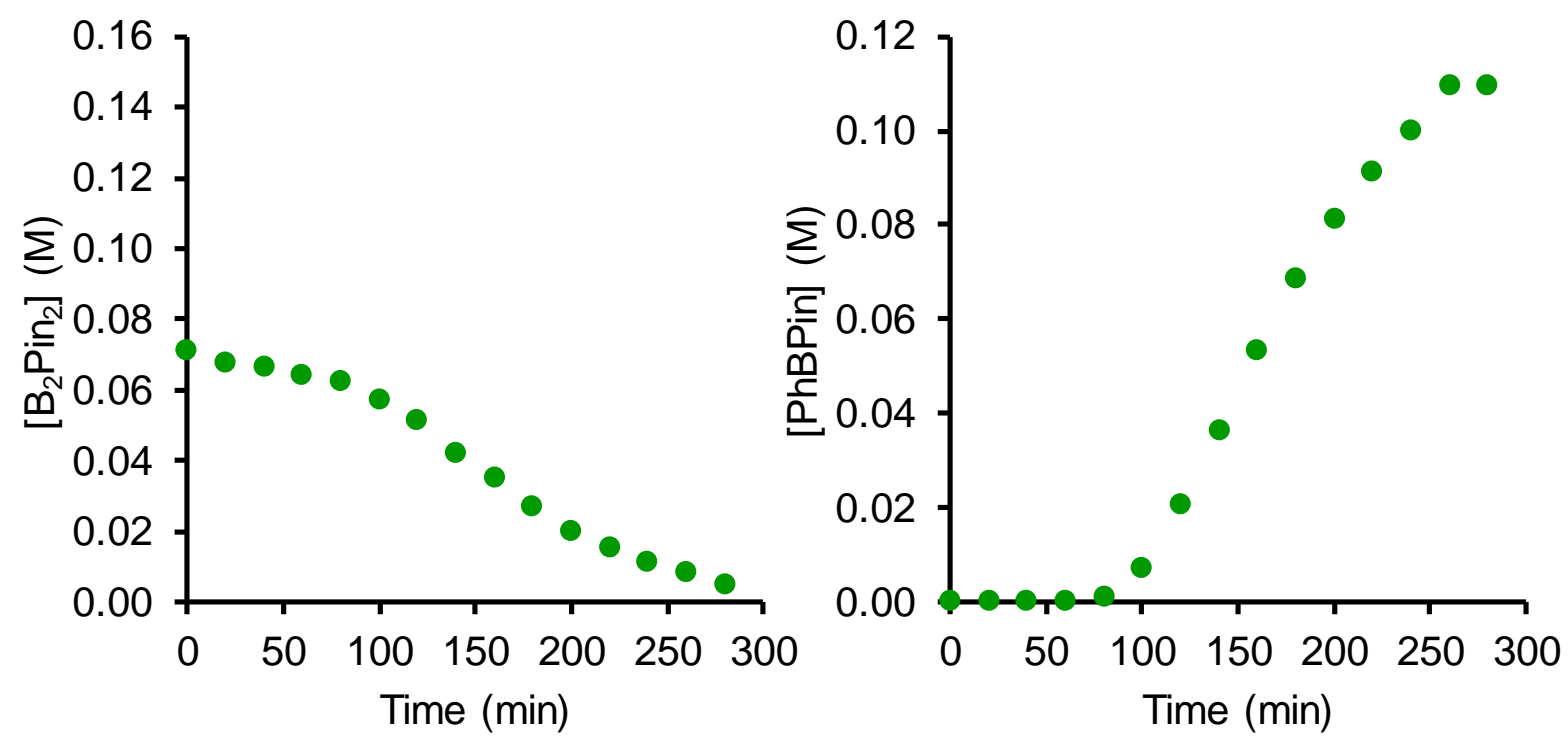

Figure S50. Benzene borylation with 2.

\section{General procedure for hot filtration test of heterogeneous benzene borylation reaction}

In a glovebox under inert atmosphere, to an oven-dried 2-5 mL Biotage microwave vial charged with stir bar was added UiO-67-mix-Ir(III) $(10 \mathrm{mg})$, anhydrous cyclohexane $(0.27 \mathrm{~mL})$, anhydrous benzene $(0.13 \mathrm{~mL}, 1.5 \mathrm{mmol}), \mathrm{B}_{2} \mathrm{Pin}_{2}$ as a stock solution in anhydrous cyclohexane $(14 \mathrm{mg}, 0.055$ $\mathrm{mmol})$, and anhydrous hexadecane $(20 \mu \mathrm{L}, 0.068 \mathrm{mmol})$ as the internal standard. The microwave vial was then sealed with a cap that was crimped. It was stirred at $80{ }^{\circ} \mathrm{C}$ at $1000 \mathrm{rpm}$ for $50 \mathrm{~min}$ using an aluminum block in the center of a Heidolph hotplate with magnetic stirring. The liquid meniscus was further wrapped in aluminum foil for insulation. After $50 \mathrm{~min}$, an aliquot was then taken with a needle and syringe as well as an outlet purge needle to relieve pressure. It was diluted with $\sim 0.2 \mathrm{~mL}$ of anhydrous cyclohexane and solids were filtered using a GE Healthcare 
Whatman $^{\mathrm{TM}}$ Mini-UniPrep ${ }^{\mathrm{TM}}$ Syringeless Filter vial, after which it was analyzed by GC-FID. The reaction was then syringe-filtered while hot into another oven-dried 2-5 mL Biotage microwave vial charged with stir bar. The vial was then sealed with a cap that was crimped. It was stirred again at $80{ }^{\circ} \mathrm{C}$ at $1000 \mathrm{rpm}$ for an additional 16 hours, after which it was analyzed by GC-FID.

Table S15. Product Distribution of Hot Filtration Test

\begin{tabular}{ccccc}
\hline Entry & $\begin{array}{c}{\left[\mathrm{B}_{2} \mathrm{Pin}_{2}\right](\mathrm{M}) \mathbf{5 0} \mathrm{min}} \\
\text { aliquot }\end{array}$ & $\begin{array}{c}\text { [PhBPin] (M) } 50 \\
\text { min aliquot }\end{array}$ & $\begin{array}{c}{\left[\mathrm{B}_{2} \mathrm{Pin}_{2}\right](\mathrm{M}) \mathbf{1 6} \mathrm{h}} \\
\text { hot filtration }\end{array}$ & $\begin{array}{c}\text { [PhBPin] (M) 16 } \mathrm{h} \\
\text { hot filtration }\end{array}$ \\
\hline 1 & 0.059 & 0.024 & 0.061 & 0.026 \\
\hline
\end{tabular}

Note, the amount of $\mathrm{B}_{2} \mathrm{Pin}_{2}$ and PhBPin stay approximately the same before and after hot filtration; minor fluctuations may be caused by release of physisorbed reagent and product from the porous material upon hot filtration.

\section{General procedure for native MOF control reaction of heterogeneous benzene borylation reaction}

In a glovebox under inert atmosphere, to an oven-dried 2-5 mL Biotage microwave vial charged with stir bar was added UiO-67-mix (1, $10 \mathrm{mg})$, anhydrous cyclohexane $(0.27 \mathrm{~mL})$, anhydrous benzene $(0.13 \mathrm{~mL}, 1.5 \mathrm{mmol}), \mathrm{B}_{2} \mathrm{Pin}_{2}$ as a stock solution in anhydrous cyclohexane (14 mg, 0.055 $\mathrm{mmol})$, and anhydrous hexadecane $(20 \mu \mathrm{L}, 0.068 \mathrm{mmol})$ as the internal standard. The microwave vial was then sealed with a cap that was crimped. It was stirred at $80{ }^{\circ} \mathrm{C}$ at $1000 \mathrm{rpm}$ for $14 \mathrm{~h}$ using an aluminum block in the center of a Heidolph hotplate with magnetic stirring. The liquid meniscus was further wrapped in aluminum foil for insulation. After $14 \mathrm{~h}$, an aliquot was then taken with a needle and syringe as well as an outlet purge needle to relieve pressure. It was diluted with $\sim 0.2 \mathrm{~mL}$ of anhydrous cyclohexane and solids were filtered using a GE Healthcare Whatman $^{\mathrm{TM}}$ Mini-UniPrep ${ }^{\mathrm{TM}}$ Syringeless Filter vial, after which it was analyzed by GC-FID.

Table S16. Product Distribution of Native MOF Control Reaction

\begin{tabular}{ccc}
\hline Entry & {$\left[\mathrm{B}_{2}\right.$ Pin $\left._{2}\right](\mathrm{M})$ after 14 $\mathrm{h}$} & {$[$ PhBPin] (M) after 14 h } \\
\hline 1 & 0.075 & 0 \\
\hline
\end{tabular}

\section{General procedure for no catalyst control reaction of benzene borylation reactions}

In a glovebox under inert atmosphere, to an oven-dried 2-5 mL Biotage microwave vial charged with stir bar was added anhydrous cyclohexane $(0.27 \mathrm{~mL})$, anhydrous benzene $(0.13 \mathrm{~mL}, 1.5$ $\mathrm{mmol}), \mathrm{B}_{2} \mathrm{Pin}_{2}$ as a stock solution in anhydrous cyclohexane (14 $\left.\mathrm{mg}, 0.055 \mathrm{mmol}\right)$, and anhydrous hexadecane $(20 \mu \mathrm{L}, 0.068 \mathrm{mmol})$ as the internal standard. The microwave vial was then sealed with a cap that was crimped. It was stirred at $80^{\circ} \mathrm{C}$ at $1000 \mathrm{rpm}$ for $14 \mathrm{~h}$ using an aluminum block in the center of a Heidolph hotplate with magnetic stirring. The liquid meniscus was further wrapped in aluminum foil for insulation. After $14 \mathrm{~h}$, an aliquot was then taken with a needle and syringe as well as an outlet purge needle to relieve pressure. It was diluted with $\sim 0.2 \mathrm{~mL}$ of anhydrous cyclohexane and solids were filtered using a GE Healthcare Whatman ${ }^{\mathrm{TM}}$ MiniUniPrep $^{\mathrm{TM}}$ Syringeless Filter vial, after which it was analyzed by GC-FID.

\section{Table S17. Product Distribution of No Catalyst Control Reaction}




\section{General procedure for kinetic monitoring of heterogeneous benzene borylation reactions}

In a glovebox under inert atmosphere, to an oven-dried 2-5 mL Biotage microwave vial charged with stir bar was added catalyst, anhydrous cyclohexane, anhydrous benzene, $\mathrm{B}_{2} \mathrm{Pin}_{2}$ as a stock solution in anhydrous cyclohexane, and anhydrous hexadecane as the internal standard. The microwave vial was then sealed with a cap that was crimped. It was stirred at $1000 \mathrm{rpm}$ using an aluminum block in the center of a Heidolph hotplate with magnetic stirring at room temperature and an initial aliquot was taken via needle and syringe using an outlet purge needle to relieve any pressure. The aliquot was diluted with $\sim 0.2 \mathrm{~mL}$ of anhydrous cyclohexane and solids were filtered using a GE Healthcare Whatman ${ }^{\mathrm{TM}}$ Mini-UniPrep ${ }^{\mathrm{TM}}$ Syringeless Filter vial. The microwave vial was then removed from the aluminum block and the temperature of the hotplate was set to $80{ }^{\circ} \mathrm{C}$. The reaction was then placed on the heated aluminum block and the liquid meniscus was further wrapped in aluminum foil for insulation. The reaction was monitored over time by GC-FID.

Table S18. Conditions for Kinetic Monitoring of Benzene Borylation

\begin{tabular}{cccccccc}
\hline Entry & $\begin{array}{c}\text { Catalyst }(\mathbf{m g}, \\
\text { targeted) }\end{array}$ & $\begin{array}{c}\text { Catalyst }(\mathbf{m g}, \\
\text { measured })\end{array}$ & $\begin{array}{c}\text { Benzene } \\
(\mathbf{M})\end{array}$ & $\begin{array}{c}\mathbf{B}_{2} \text { Pin }_{2} \\
\mathbf{( M ,} \\
\text { targeted) }\end{array}$ & $\begin{array}{c}\mathbf{B}_{2} \text { Pin }_{2}(\mathbf{M}, \\
\text { measured } \\
\text { by GC-FID) }\end{array}$ & $\begin{array}{c}\text { Hexadecane } \\
(\mathbf{M})\end{array}$ & $\begin{array}{c}\text { Total } \\
\text { Volume } \\
(\mathbf{m L})\end{array}$ \\
\hline 1 & 40 & 40.3 & 0.22 & 0.073 & 0.079 & 0.023 & 3.02 \\
2 & 40 & 40.9 & 0.48 & 0.073 & 0.072 & 0.023 & 3.02 \\
3 & 40 & 39.9 & 0.96 & 0.073 & 0.074 & 0.023 & 3.02 \\
4 & 40 & 40.3 & 1.93 & 0.073 & 0.077 & 0.023 & 3.02 \\
5 & 40 & 40.6 & 0.48 & 0.037 & 0.041 & 0.023 & 3.02 \\
6 & 40 & 40.2 & 0.48 & 0.018 & 0.018 & 0.023 & 3.02 \\
7 & 40 & 40.6 & 0.48 & 0.146 & 0.139 & 0.023 & 3.02 \\
8 & 20 & 20.3 & 1.93 & 0.073 & 0.071 & 0.023 & 3.02 \\
9 & 80 & 80.4 & 1.93 & 0.073 & 0.070 & 0.023 & 3.02 \\
\hline
\end{tabular}

$$
\text { Mass Balance }(\%)=\frac{\left[\mathrm{B}_{2} \mathrm{Pin}_{2}\right]+\frac{1}{2}[\mathrm{PhBPin}]}{\left[\mathrm{B}_{2} \mathrm{Pin}_{2}\right]_{\text {initial }}} \times 100
$$

Equation 1. Calculation of mass balance as a percentage based on limiting reagent $\mathrm{B}_{2} \mathrm{Pin}_{2}$ for benzene borylation kinetic plots. 


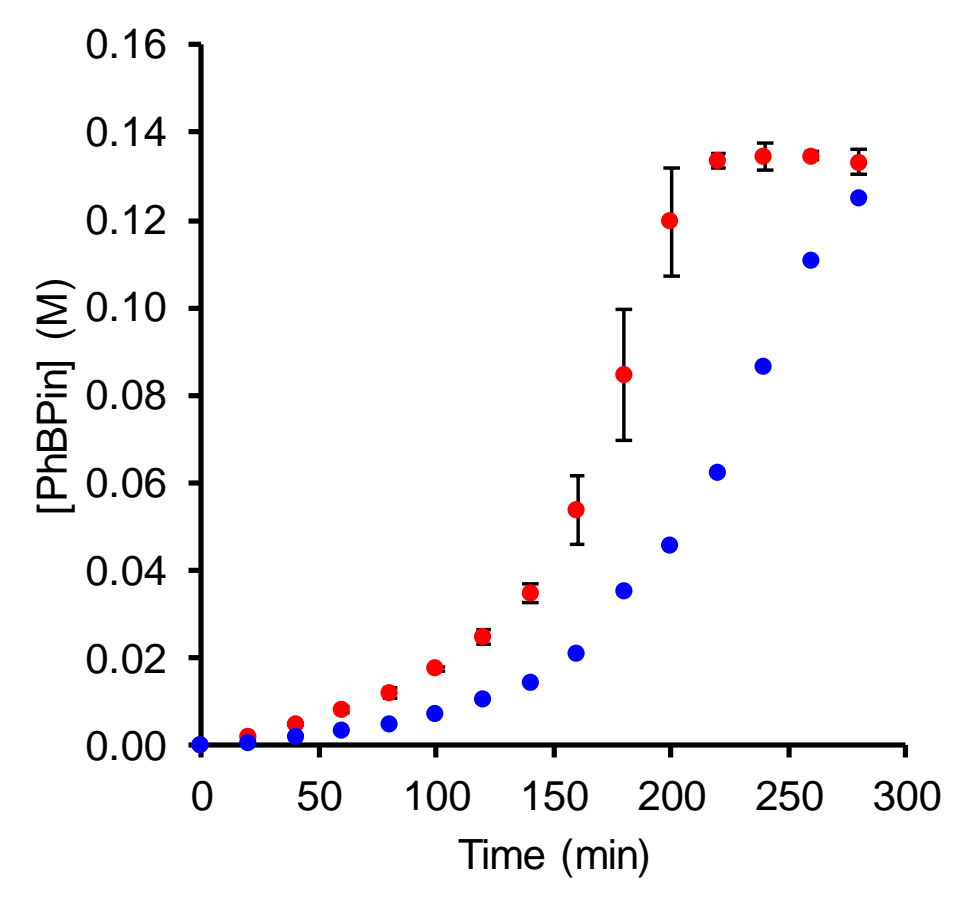

- Catalyst Batch $1 \quad$ Catalyst Batch 2

Figure S51. A comparison of benzene borylation with two batches of catalyst. Catalyst batch 1 represents the kinetic plot of [PhBPin] formation from Table S19, entries 1-3 with error bars representing standard deviation (sample) of the triplicate runs. Catalyst batch 2 represents a reaction run with a different catalyst batch under conditions listed in Table S18, entry 2 . This plot shows that error within the same catalyst batch is low however the instantaneous rate can differ between batches.

$0.22 \mathrm{M}$ [Benzene]

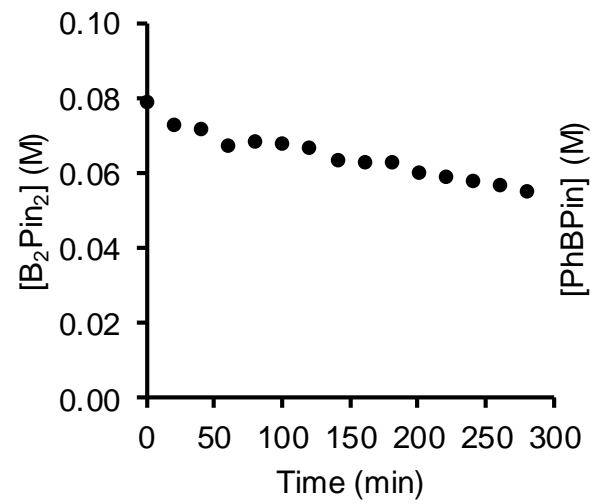

$0.22 \mathrm{M}$ [Benzene]

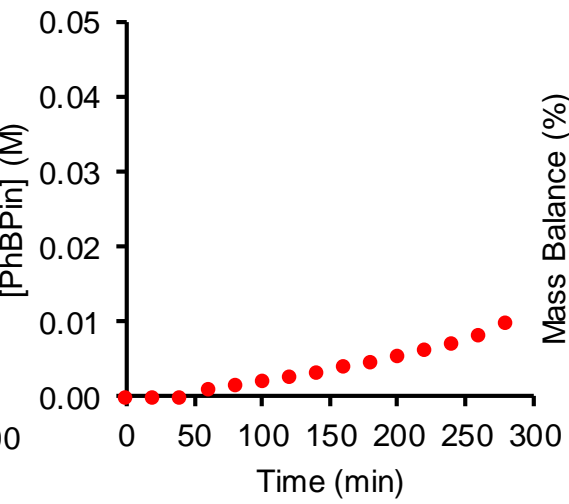

$0.22 \mathrm{M}$ [Benzene]

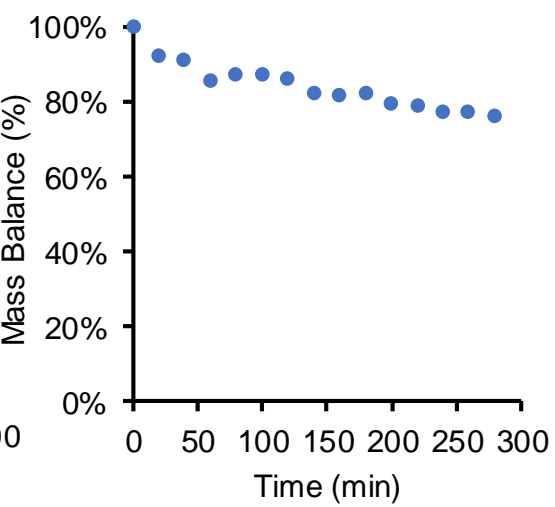

Figure S52. Kinetic plots for Table S18, Entry 1. 

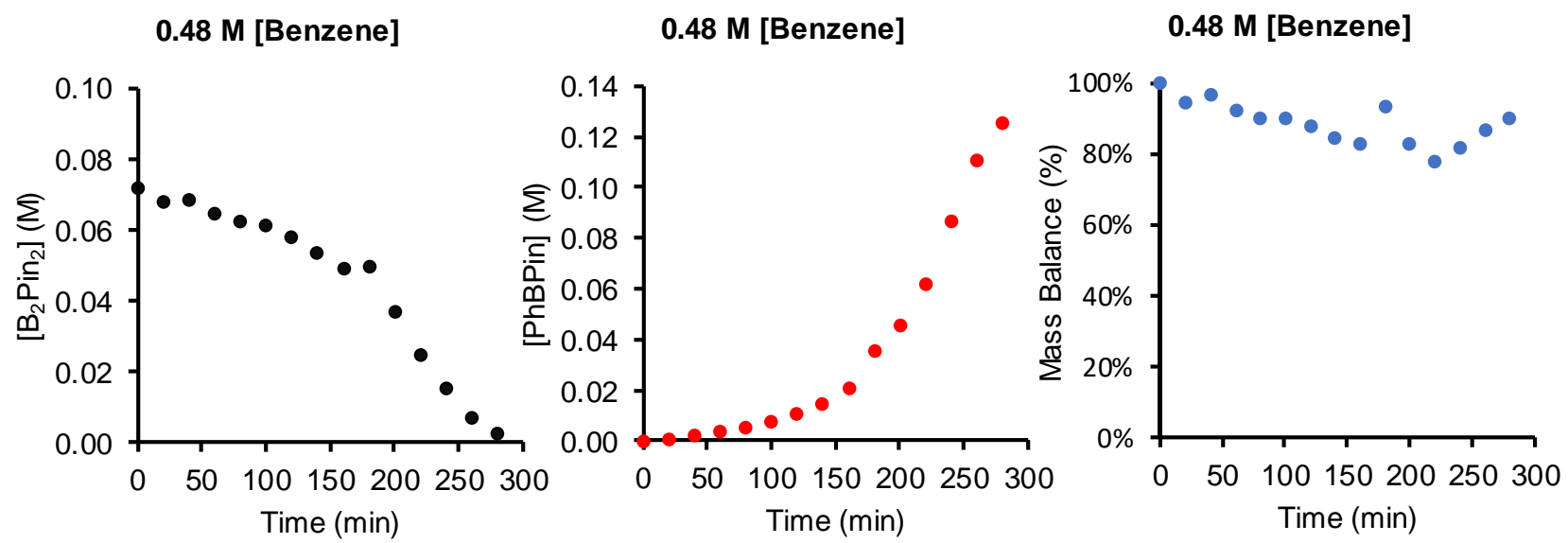

Figure S53. Kinetic plots for Table S18, Entry 2.

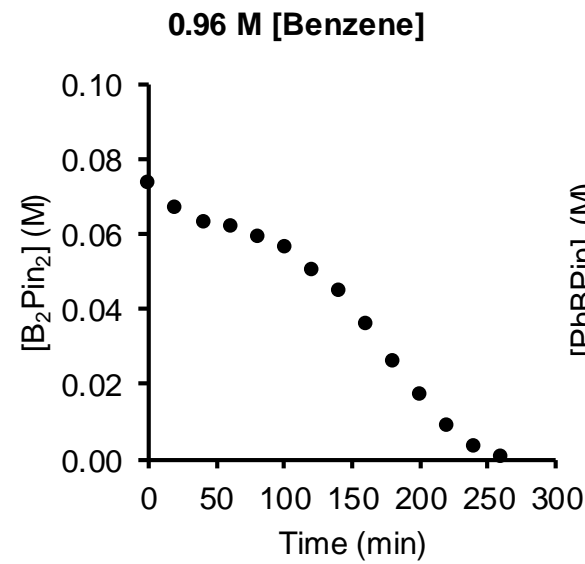

0.96 M [Benzene]

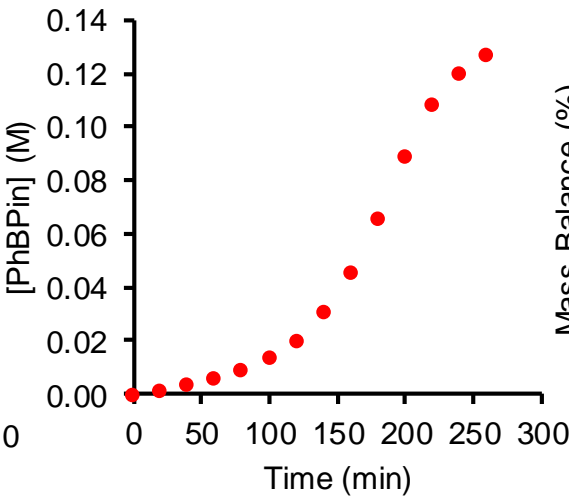

0.96 M [Benzene]

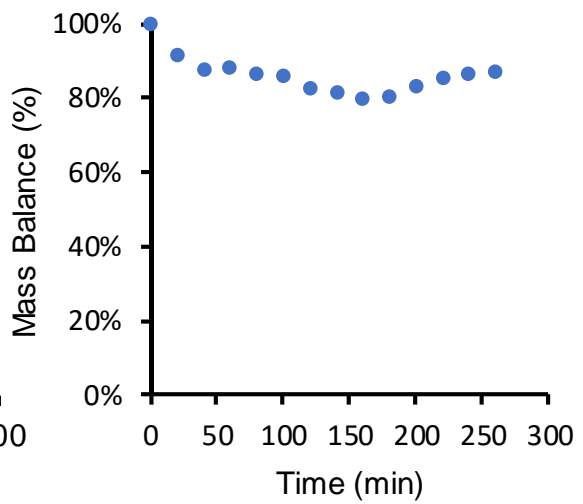

Figure S54. Kinetic plots for Table S18, Entry 3.

1.93 M [Benzene]

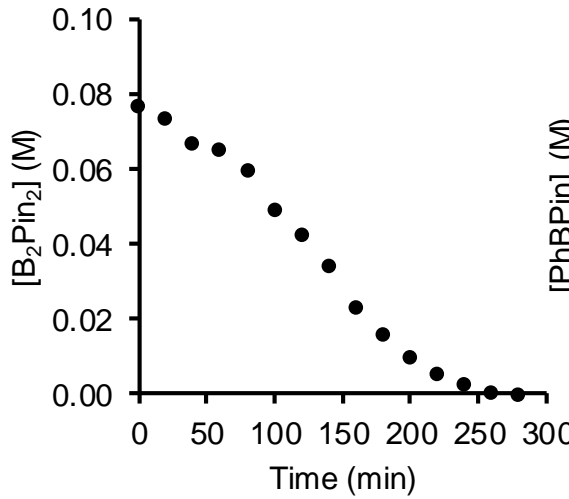

1.93 M [Benzene]

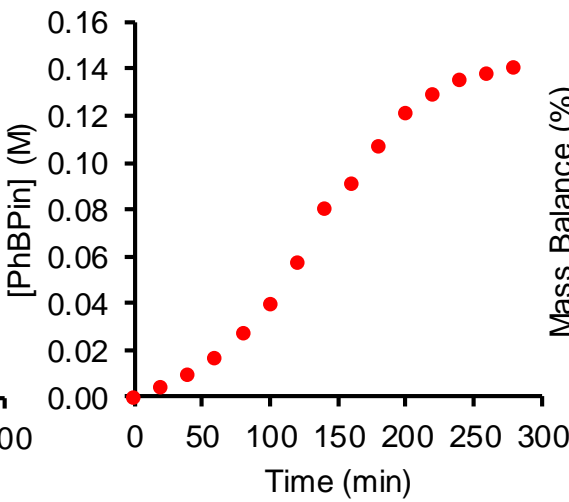

1.93 M [Benzene]

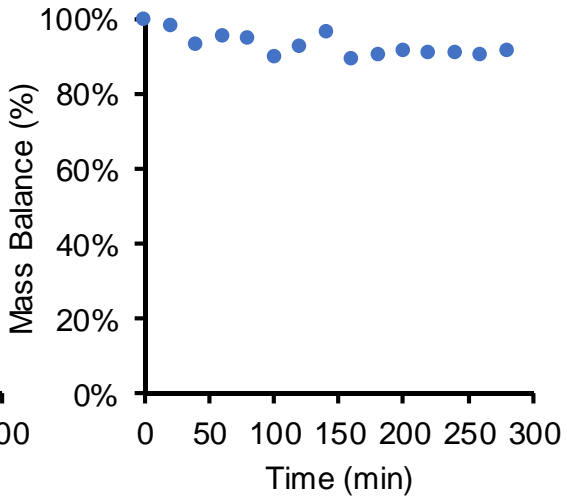

Figure S55. Kinetic plots for Table S18, Entry 4. 
$0.037 \mathrm{M}\left[\mathrm{B}_{2} \mathrm{Pin}_{2}\right]$

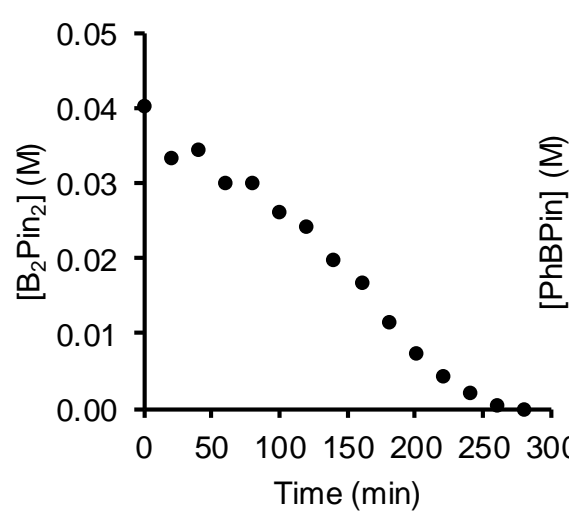

$0.037 \mathrm{M}\left[\mathrm{B}_{2} \mathrm{Pin}_{2}\right]$

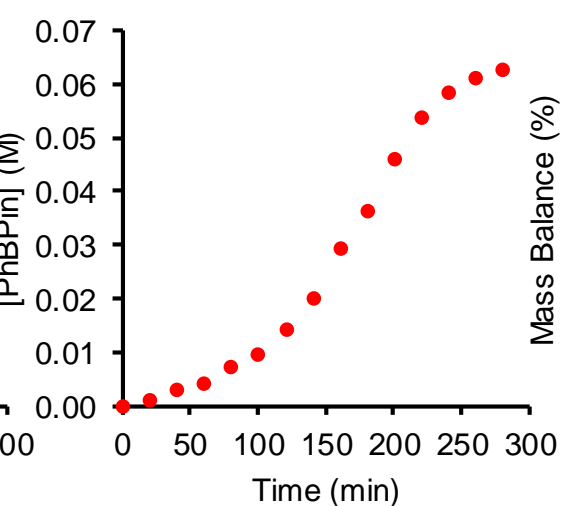

$0.037 \mathrm{M}\left[\mathrm{B}_{2} \mathrm{Pin}_{2}\right]$

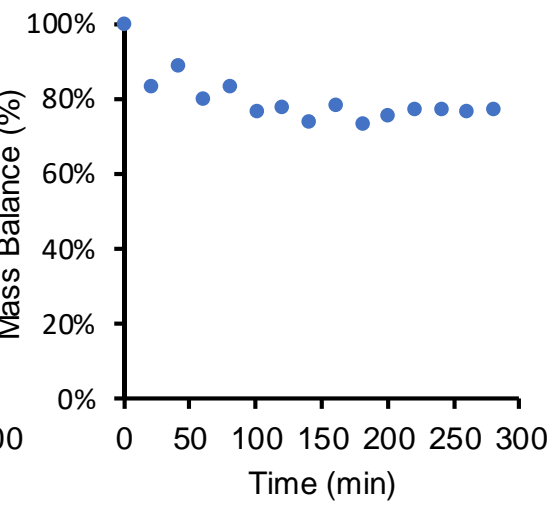

Figure S56. Kinetic plots for Table S18, Entry 5.
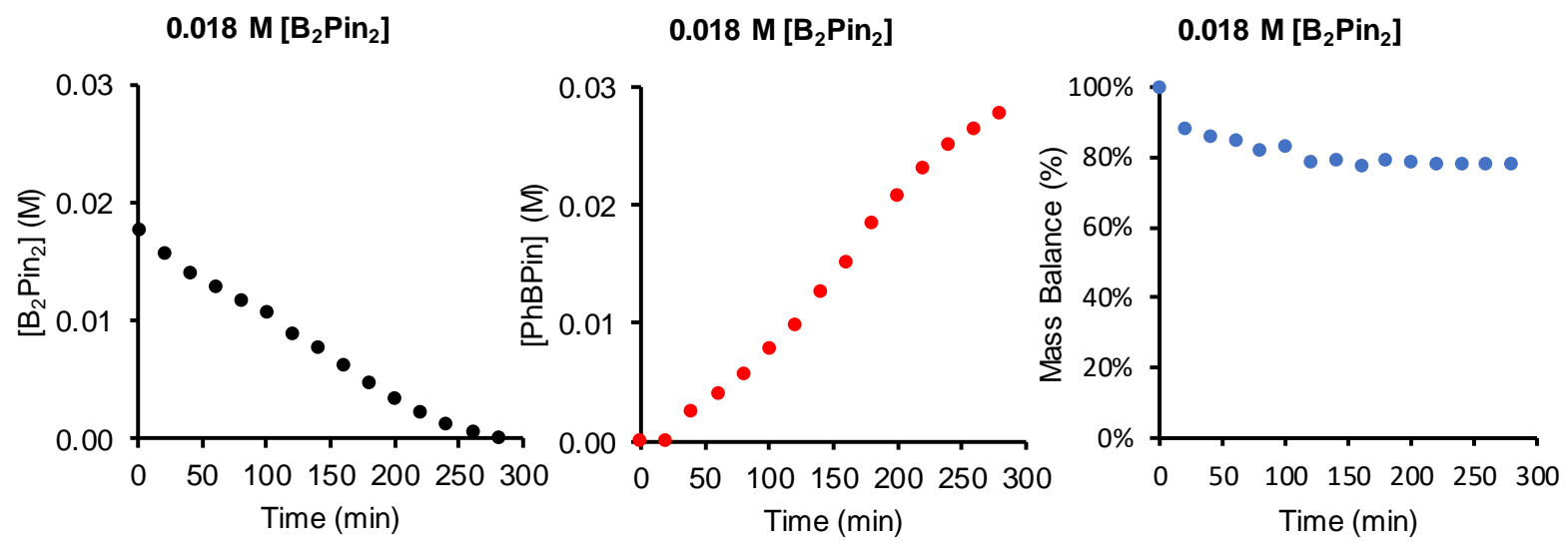

Figure S57. Kinetic plots for Table S18, Entry 6.

$0.146 \mathrm{M}\left[\mathrm{B}_{2} \mathrm{Pin}_{2}\right]$

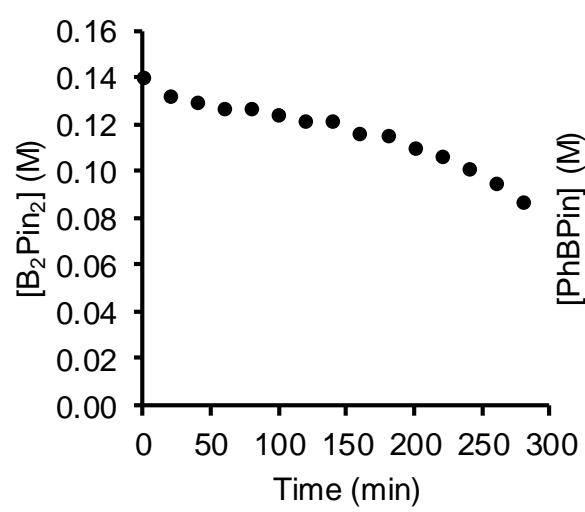

$0.146 \mathrm{M}\left[\mathrm{B}_{2} \mathrm{Pin}_{2}\right]$

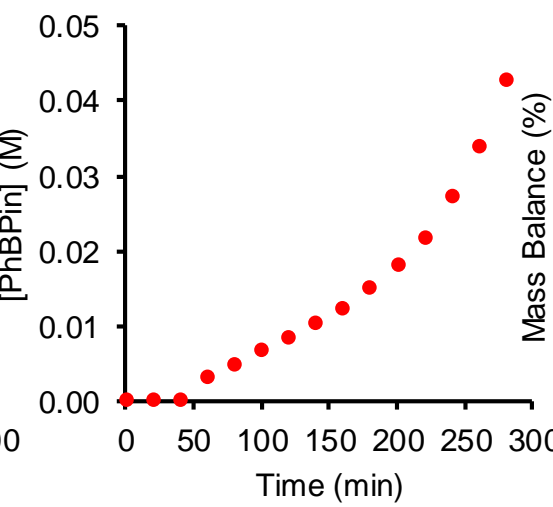

$0.146 \mathrm{M}\left[\mathrm{B}_{2} \mathrm{Pin}_{2}\right]$

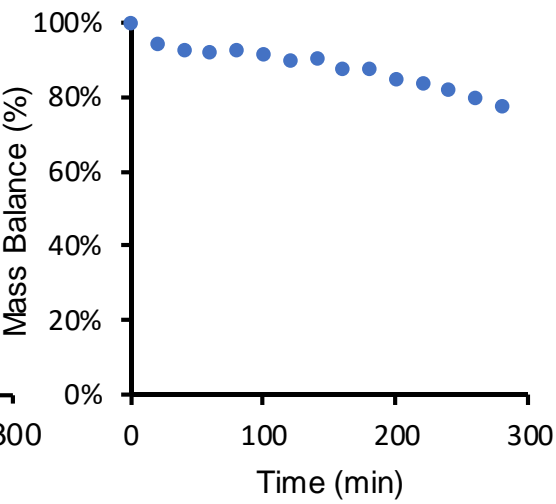

Figure S58. Kinetic plots for Table S18, Entry 7. 
$20 \mathrm{mg}$ Catalyst

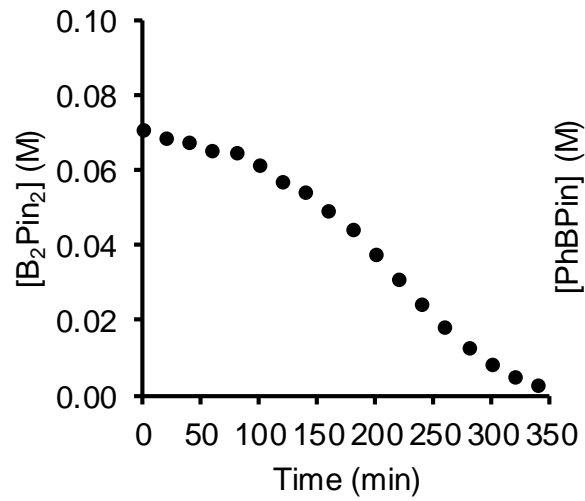

$20 \mathrm{mg}$ Catalyst

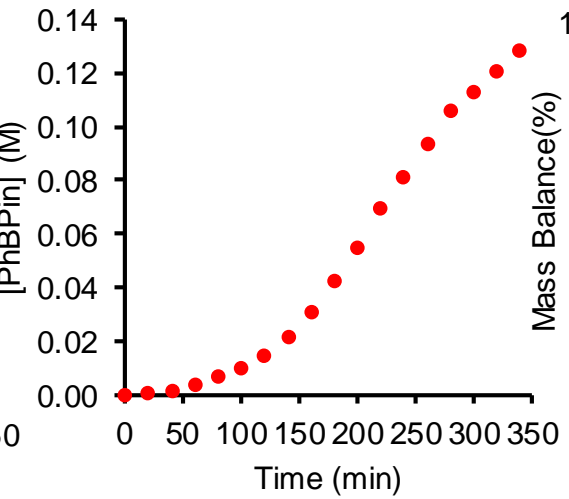

20 mg Catalyst

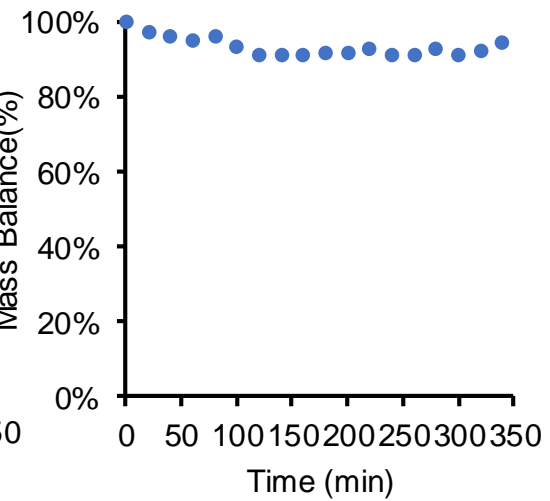

Figure S59. Kinetic plots for Table S18, Entry 8.
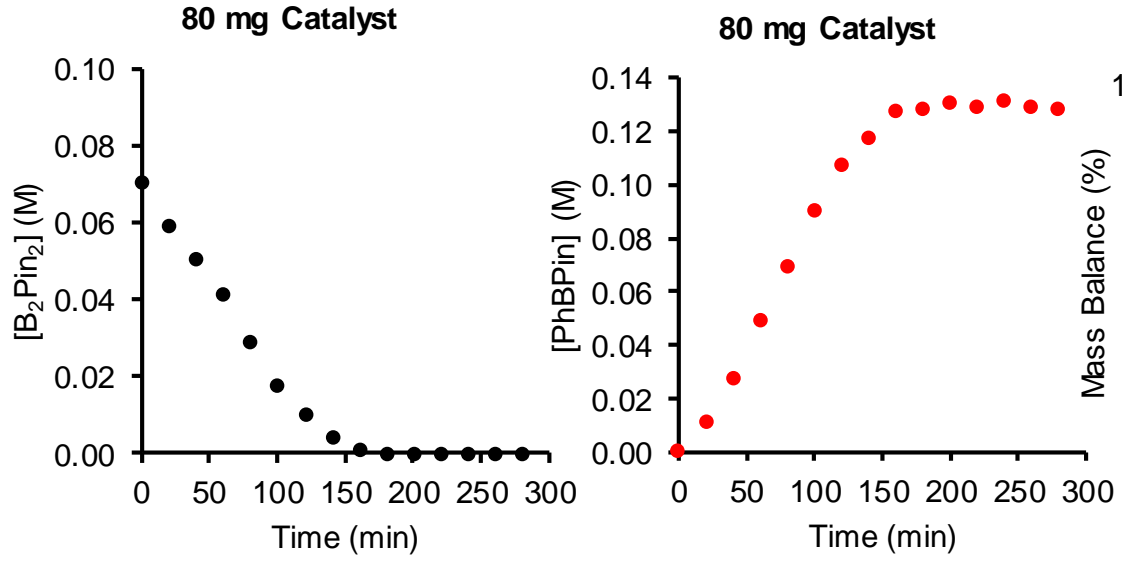

$80 \mathrm{mg}$ Catalyst

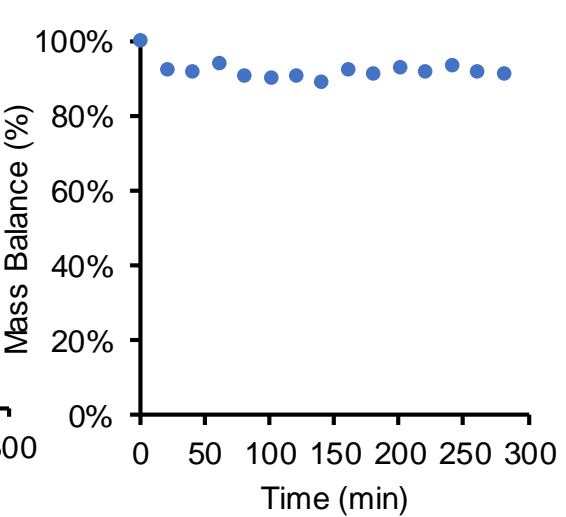

Figure S60. Kinetic plots for Table S18, Entry 9.

$$
\sum_{a=1}^{n}\left(\frac{\left(\left[\mathrm{B}_{2} \mathrm{Pin}_{2}\right]_{a}+\left[\mathrm{B}_{2} \mathrm{Pin}_{2}\right]_{a-1}\right)}{2}\right)^{x} \times\left(\mathrm{t}_{a}-\mathrm{t}_{a-1}\right)
$$

Equation 2. Mathematical expression to obtain each value of the normalized time scale for VTNA to deduce rate dependence on $\left[\mathrm{B}_{2} \mathrm{Pin}_{2}\right]$ in the borylation reaction. An overlay of the kinetic data can be obtained when a normalized time scale is generated in place of the time axis. This normalization is represented by the time integral of $\left[\mathrm{B}_{2} \mathrm{Pin}_{2}\right]$ raised to the appropriate exponent " $x$ ", which represents the order with respect to $\left[\mathrm{B}_{2} \mathrm{Pin}_{2}\right]$. 


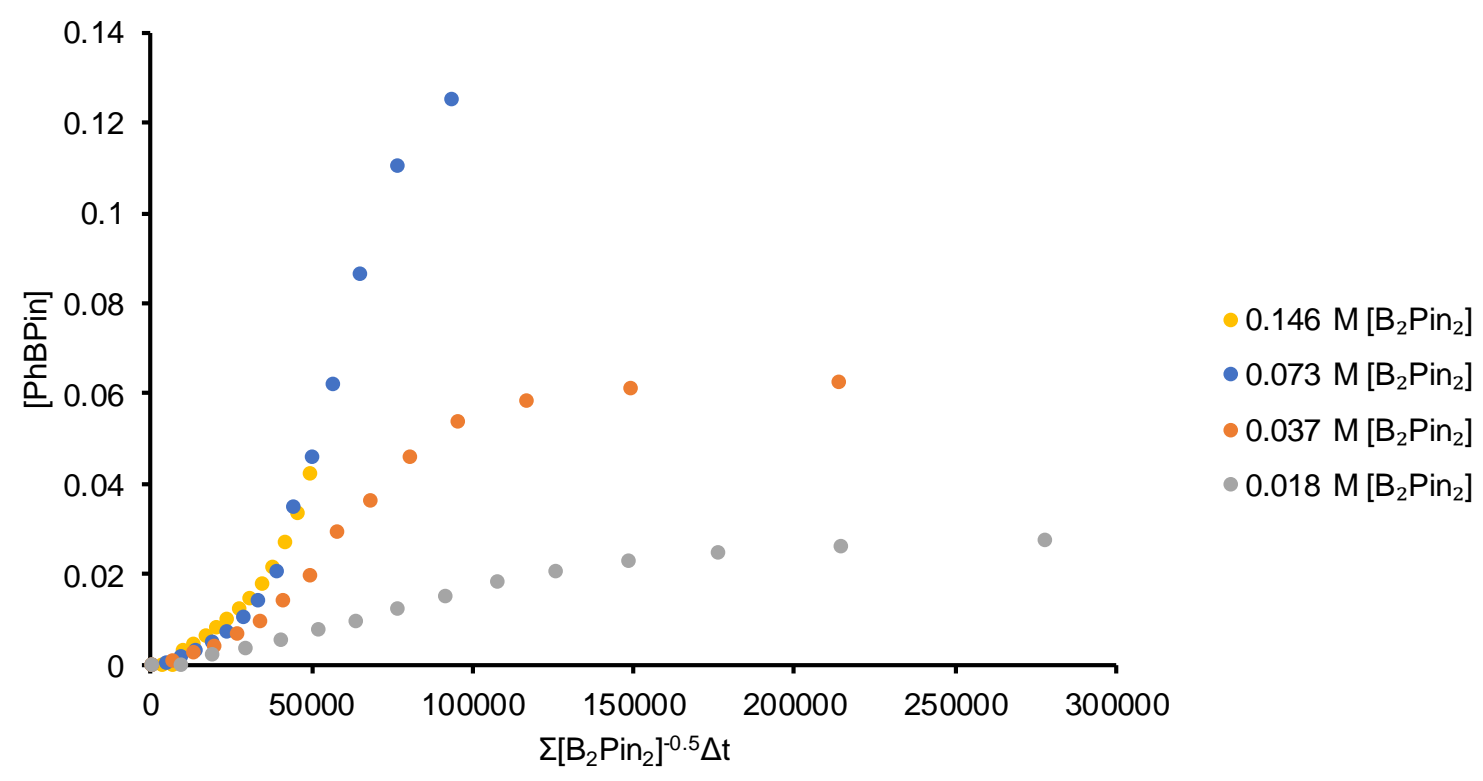

Figure S61. VTNA plots for determination of rate dependence on $\mathrm{B}_{2} \mathrm{Pin}_{2}$ (Table S18. Entries 2, $5,6,7),-0.5$ represents the plot fit to -0.5 order.

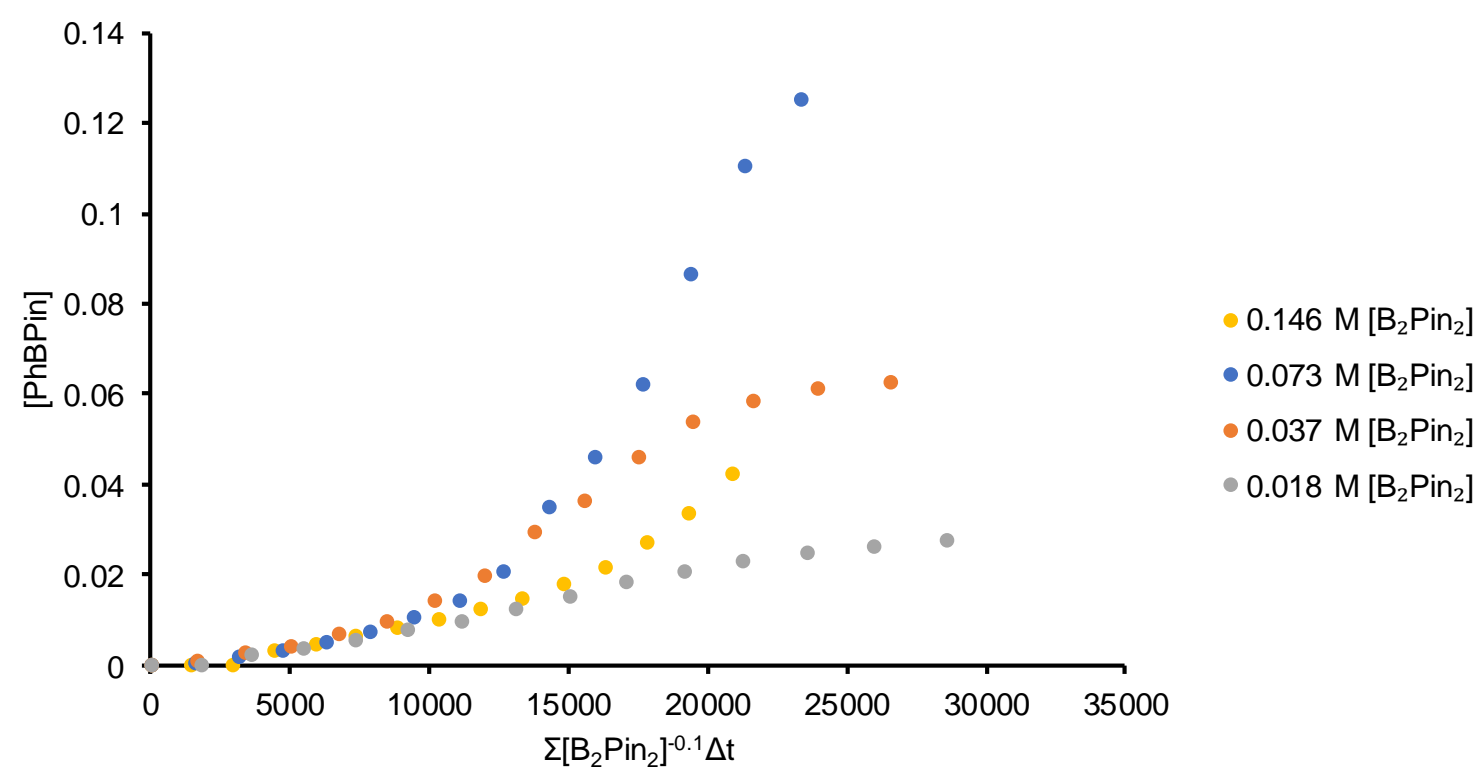

Figure S62. VTNA plots for determination of rate dependence on $\mathrm{B}_{2} \mathrm{Pin}_{2}$ (Table S18. Entries 2, $5,6,7),-0.1$ represents the plot fit to -0.1 order. 


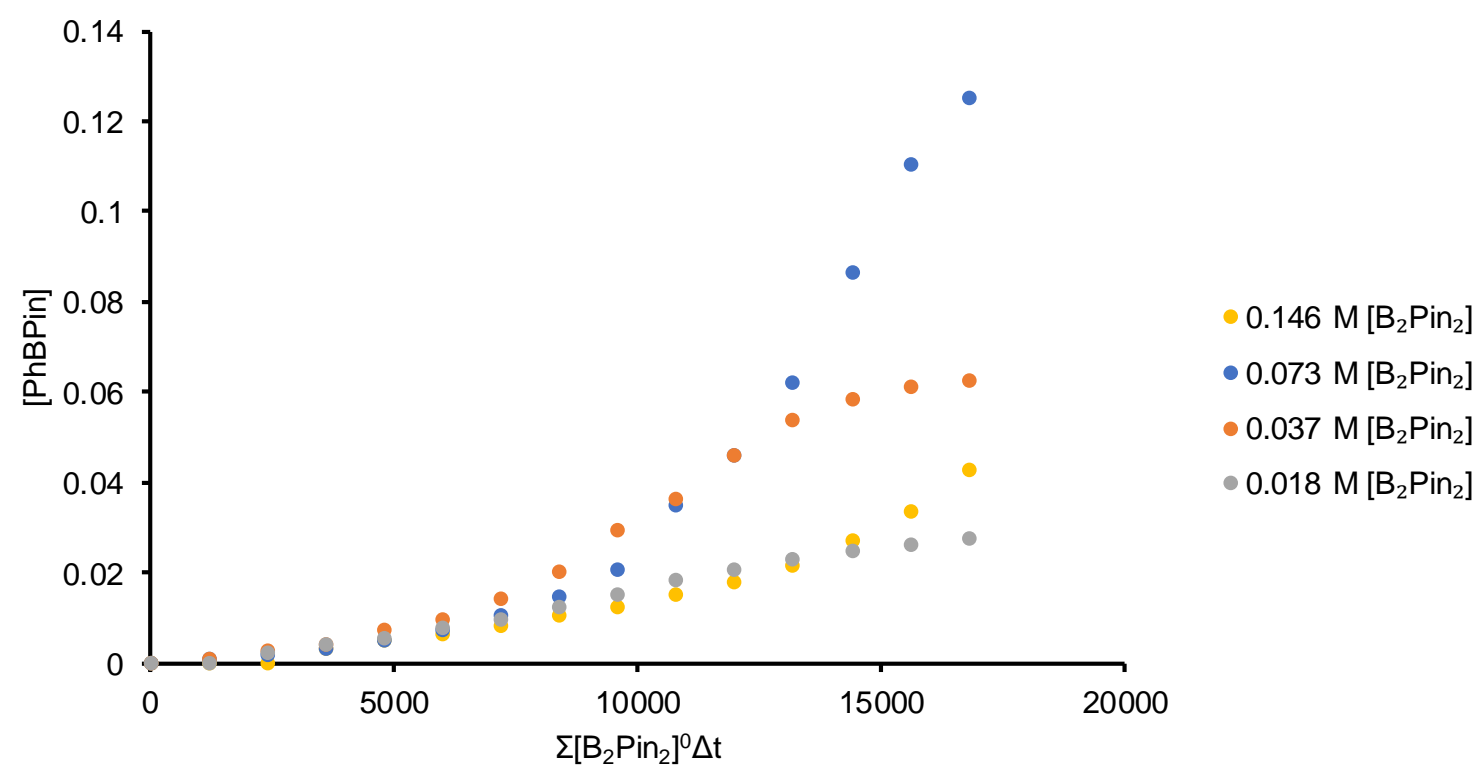

Figure S63. VTNA plots for determination of rate dependence on $\mathrm{B}_{2} \mathrm{Pin}_{2}$ (Table S18. Entries 2, $5,6,7), 0$ represents the plot fit to 0 order.

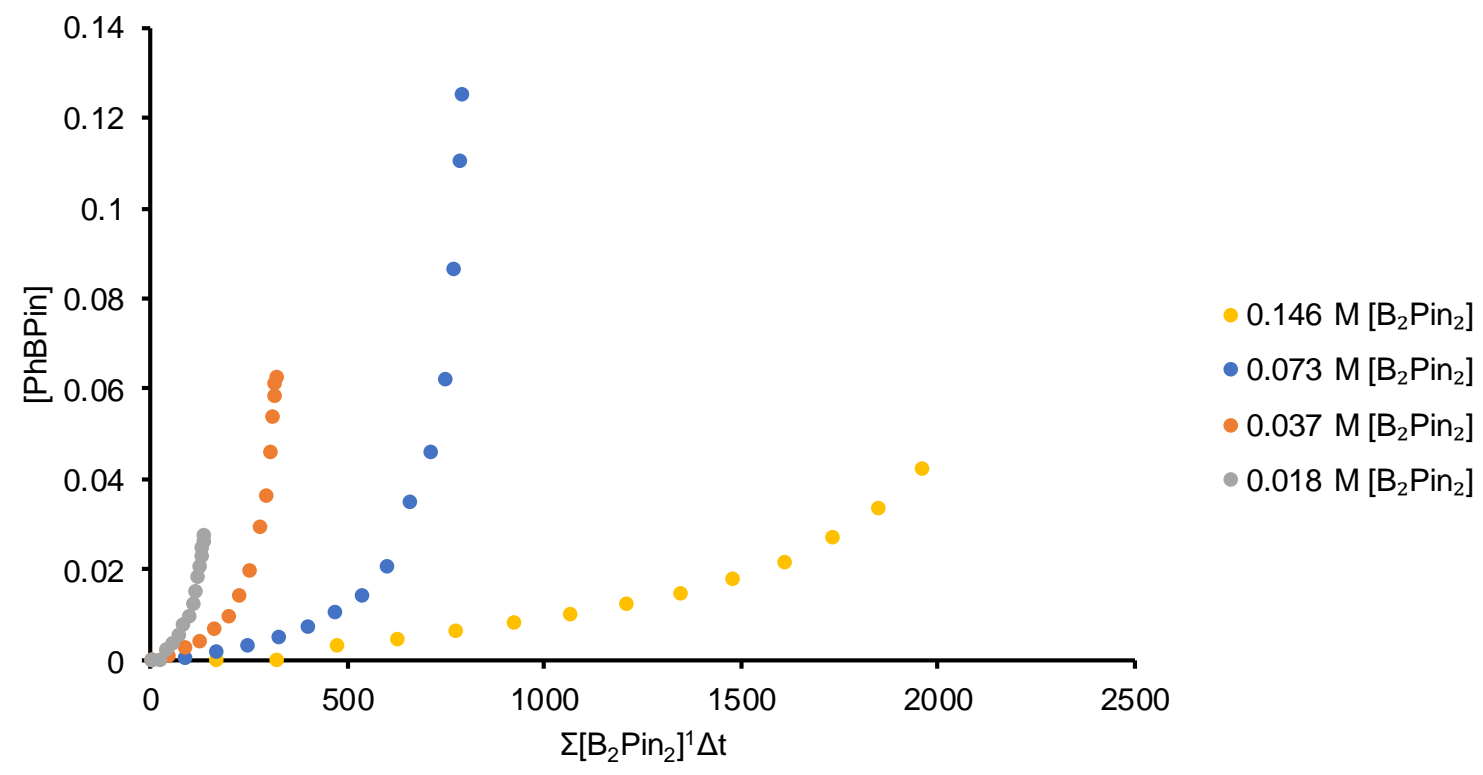

Figure S64. VTNA plots for determination of rate dependence on $\mathrm{B}_{2} \mathrm{Pin}_{2}$ (Table S18. Entries 2, $5,6,7), 1$ represents the plot fit to 1 order. 


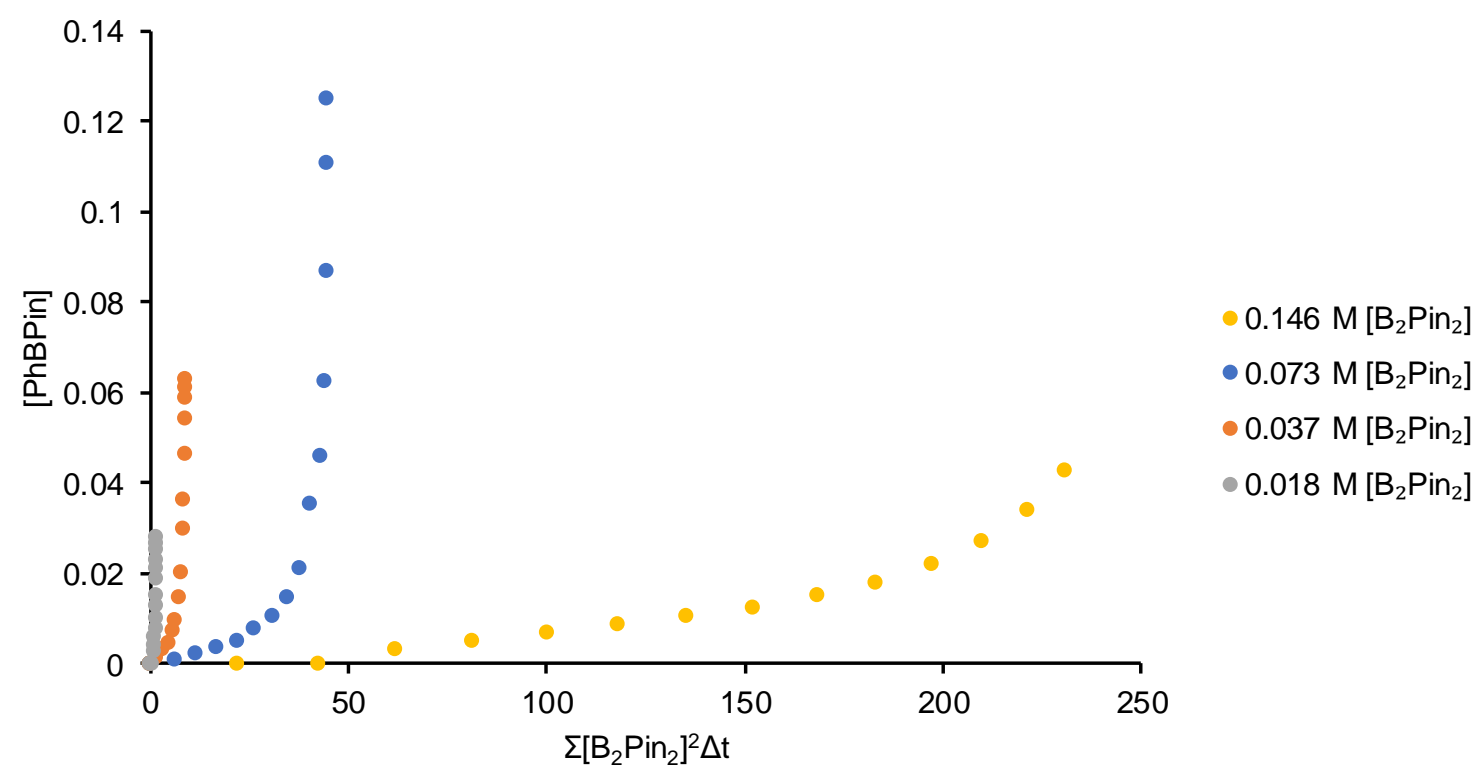

Figure S65. VTNA plots for determination of rate dependence on $\mathrm{B}_{2} \mathrm{Pin}_{2}$ (Table S18. Entries 2, $5,6,7), 2$ represents the plot fit to 2 order.

$$
\begin{gathered}
\sum_{a=1}^{n}\left(\frac{\left([\text { Benzene }]_{a}+[\text { Benzene }]_{a-1}\right)}{2}\right)^{x} \times\left(\mathrm{t}_{a}-\mathrm{t}_{a-1}\right) \\
{[\text { Benzene }]=[\text { Benzene }]_{\text {initial }}-[\text { PhBPin }]}
\end{gathered}
$$

Equation 3. Mathematical expression to obtain each value of the normalized time scale for VTNA to deduce rate dependence on [Benzene] in the borylation reaction. An overlay of the kinetic data can be obtained when a normalized time scale is generated in place of the time axis. This normalization is represented by the time integral of [Benzene] raised to the appropriate exponent " $x$ ", which represents the order with respect to [Benzene]. To calculate [Benzene] for each time point, [PhBPin] at the desired timepoint is subtracted from [Benzene] $]_{\text {initial, }}$ due to their $1: 1$ reaction stoichiometry. 


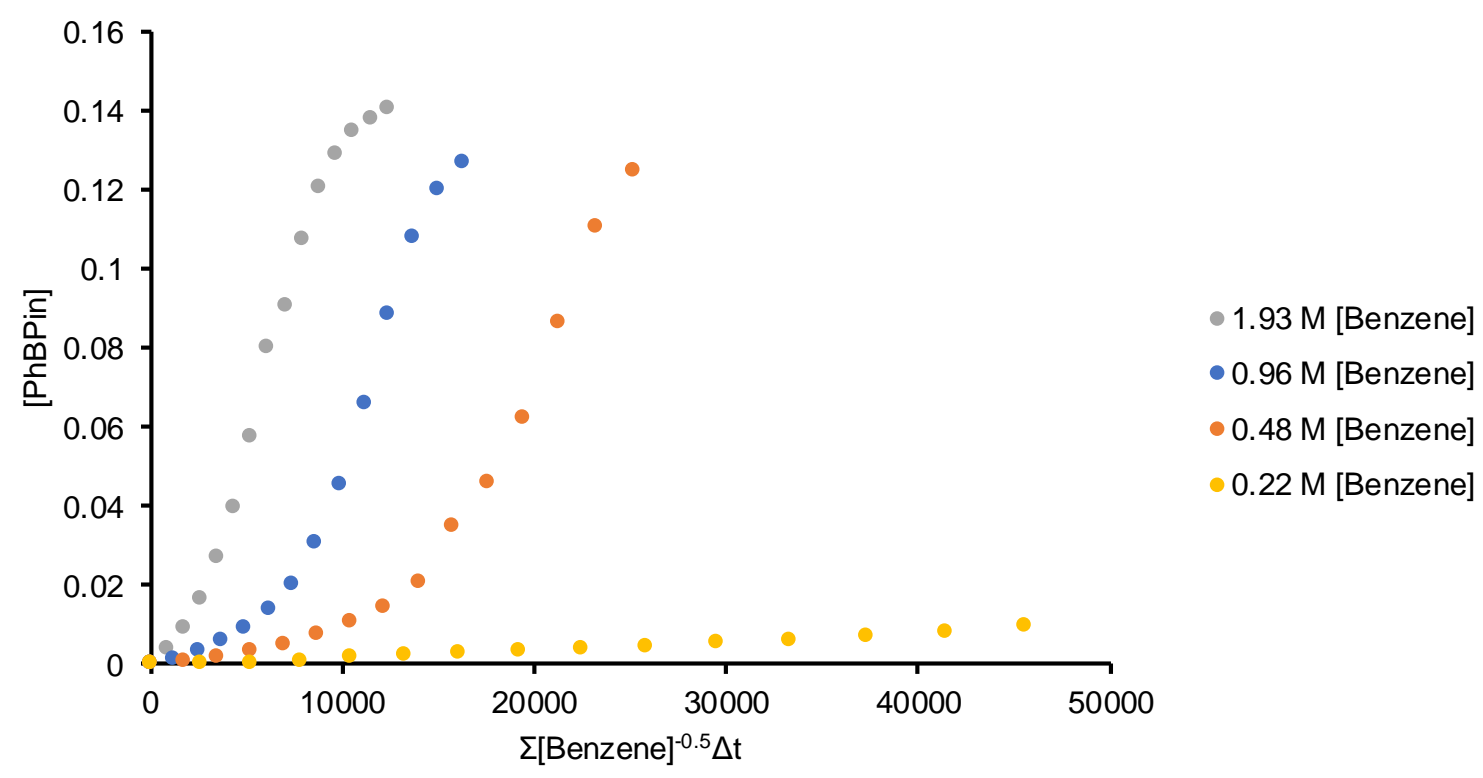

Figure S66. VTNA plots for determination of rate dependence on Benzene (Table S18. Entries $1,2,3,4),-0.5$ represents the plot fit to -0.5 order.

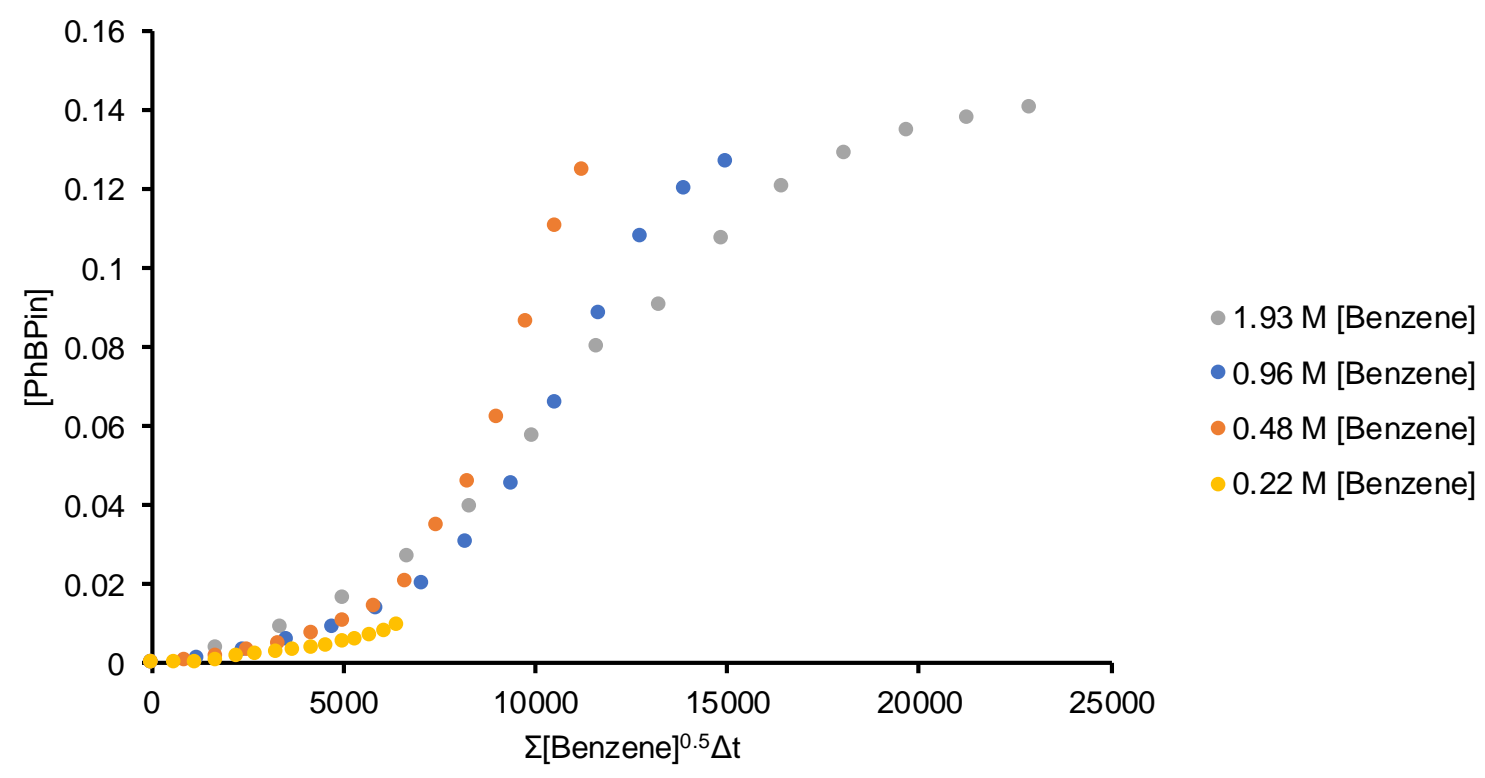

Figure S67. VTNA plots for determination of rate dependence on Benzene (Table S18. Entries $1,2,3,4), 0.5$ represents the plot fit to 0.5 order. 


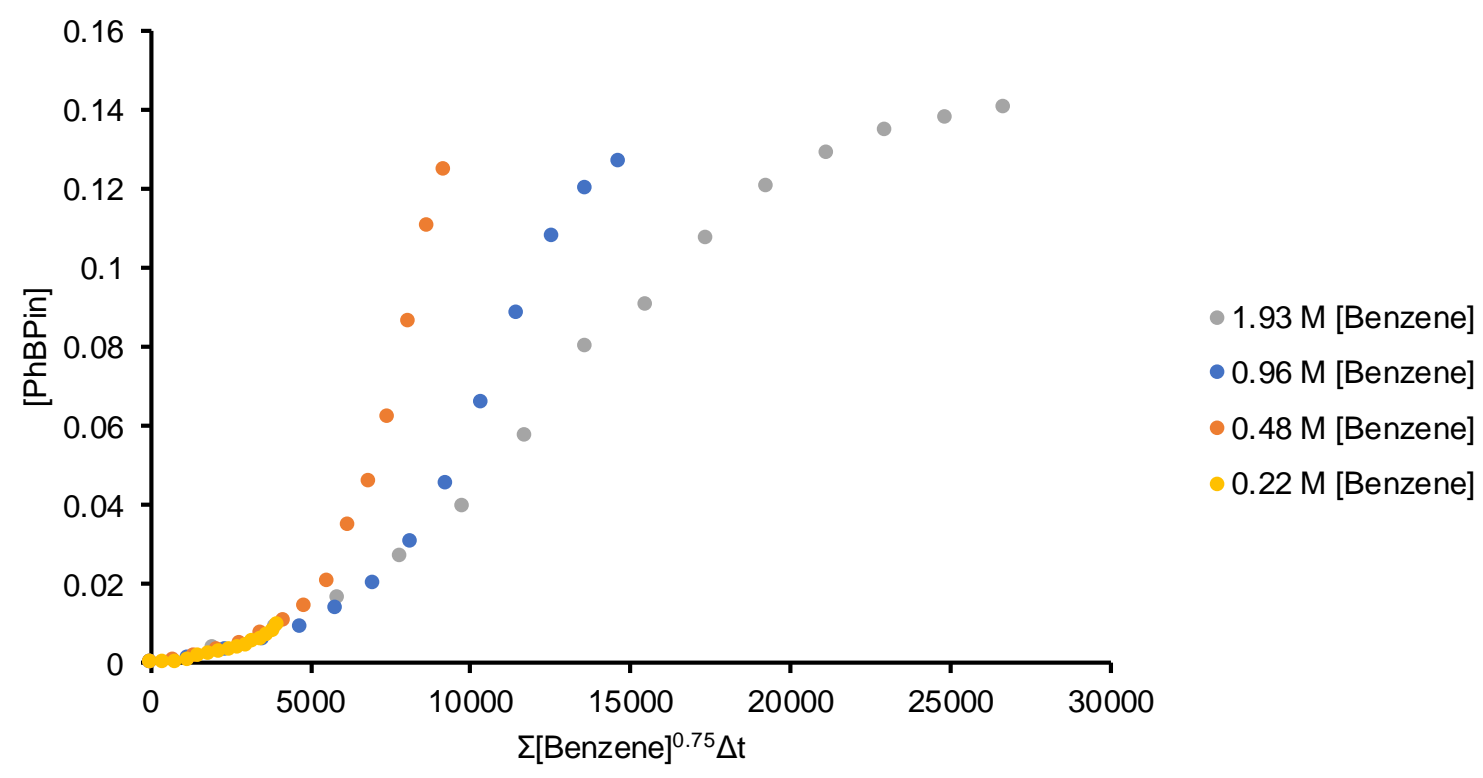

Figure S68. VTNA plots for determination of rate dependence on Benzene (Table S18. Entries $1,2,3,4), 0.75$ represents the plot fit to 0.75 order.

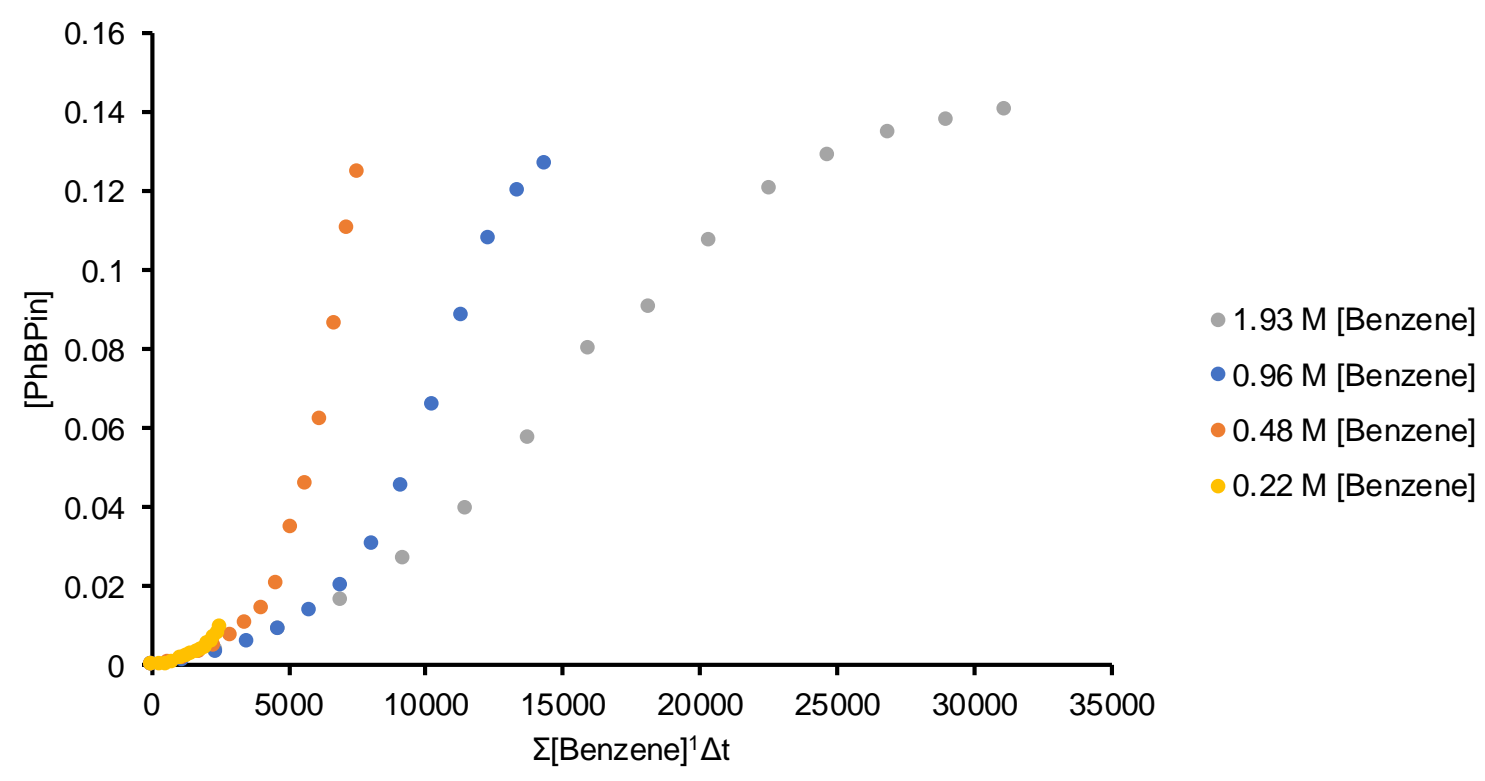

Figure S69. VTNA plots for determination of rate dependence on Benzene (Table S18. Entries $1,2,3,4), 1$ represents the plot fit to 1 order. 


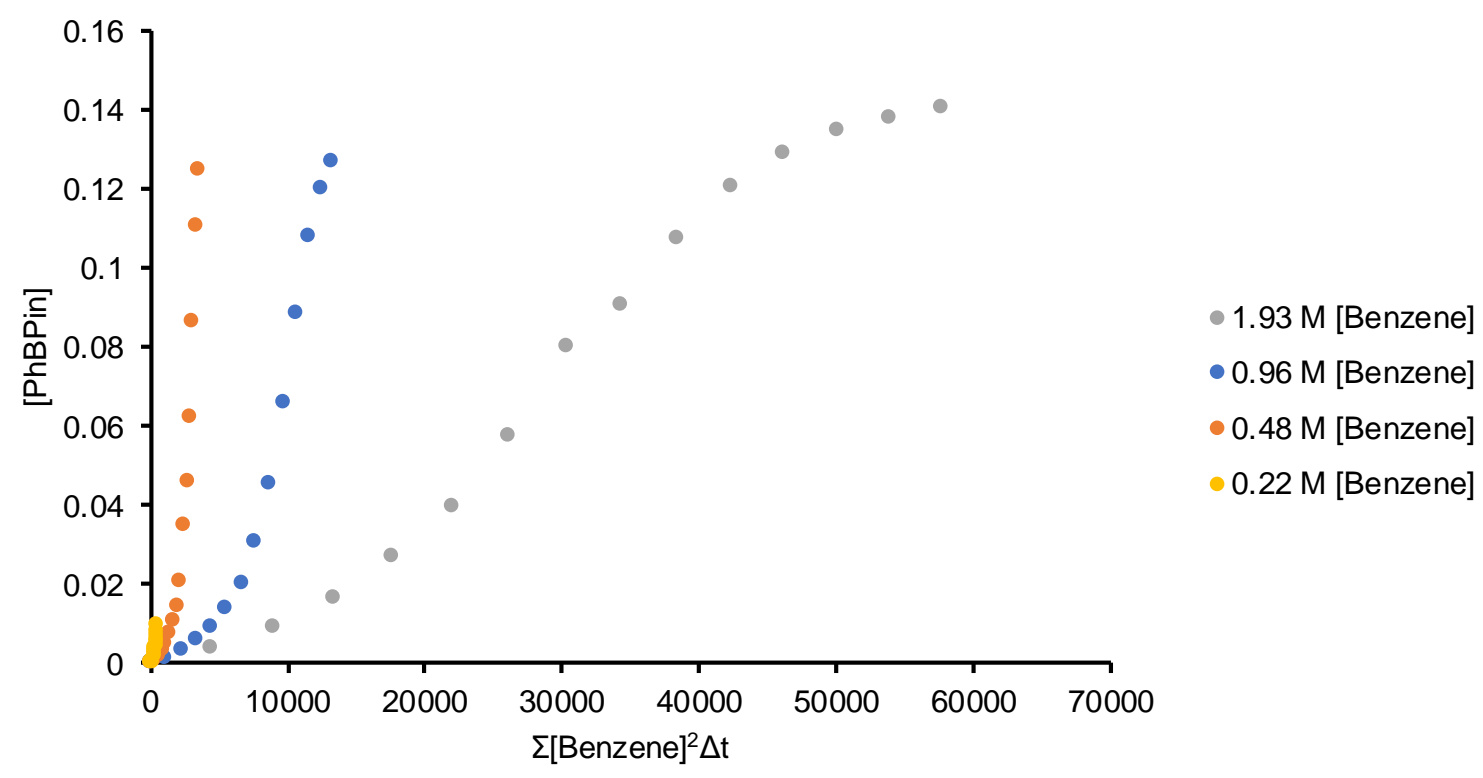

Figure S70. VTNA plots for determination of rate dependence on Benzene (Table S18. Entries $1,2,3,4), 2$ represents the plot fit to 2 order.

$$
\begin{gathered}
\left([\text { Catalyst }]_{a}\right)^{x} \times\left(\mathrm{t}_{a}\right) \\
{[\text { Catalyst }]=\frac{\mathrm{mmol} \text { Ir in Catalyst }}{\mathrm{mL} \text { of reaction }}}
\end{gathered}
$$

Equation 4. Mathematical expression to obtain each value of the normalized time scale for VTNA to deduce rate dependence on [Catalyst] in the borylation reaction. An overlay of the kinetic data can be obtained when a normalized time scale is generated in place of the time axis. This normalization is represented by the [Catalyst] raised to the appropriate exponent " $x$ ", which represents the order with respect to [Catalyst] multiplied by the time. To calculate [Catalyst], the $\mathrm{mmol}$ of Ir in the catalyst are calculated using values found by ICP-OES and this is divided by the volume of the reaction. 


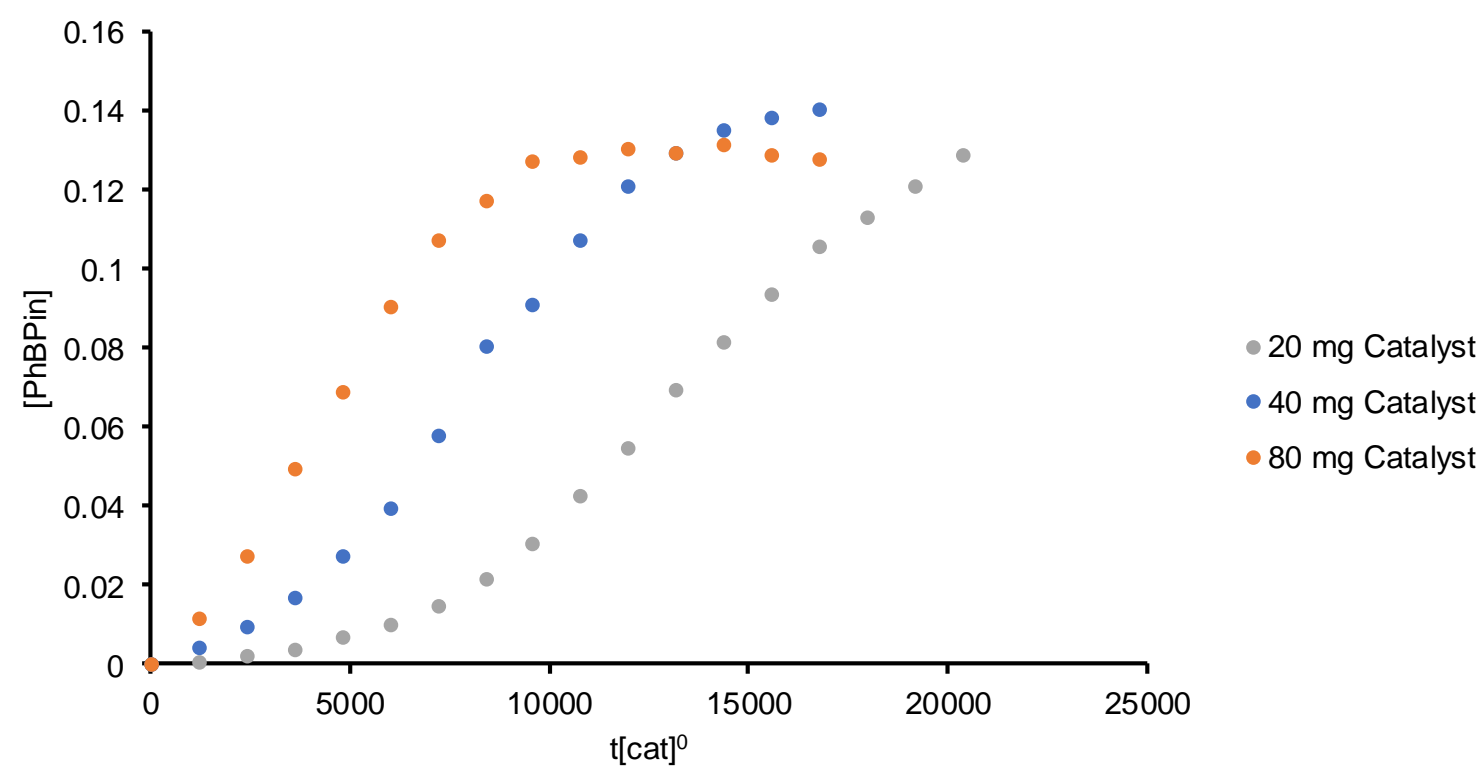

Figure S71. VTNA plots for determination of rate dependence on Catalyst (Table S18. Entries 4, 8, 9), 0 represents the plot fit to 0 order.

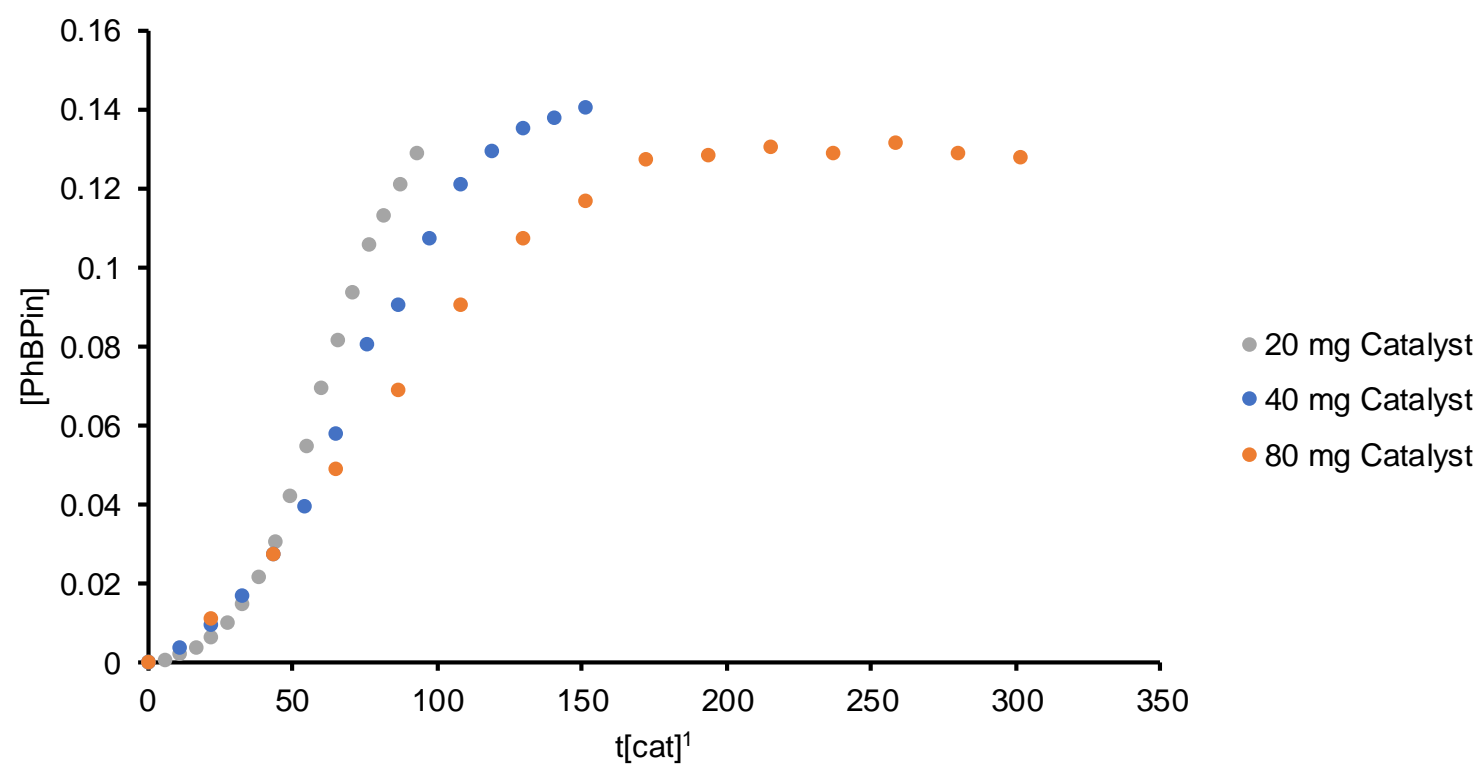

Figure S72. VTNA plots for determination of rate dependence on Catalyst (Table S18. Entries 4, $8,9), 1$ represents the plot fit to 1 order. 


\section{$\mathrm{B}_{2} \mathrm{Pin}_{2}$ Calibration Curve}

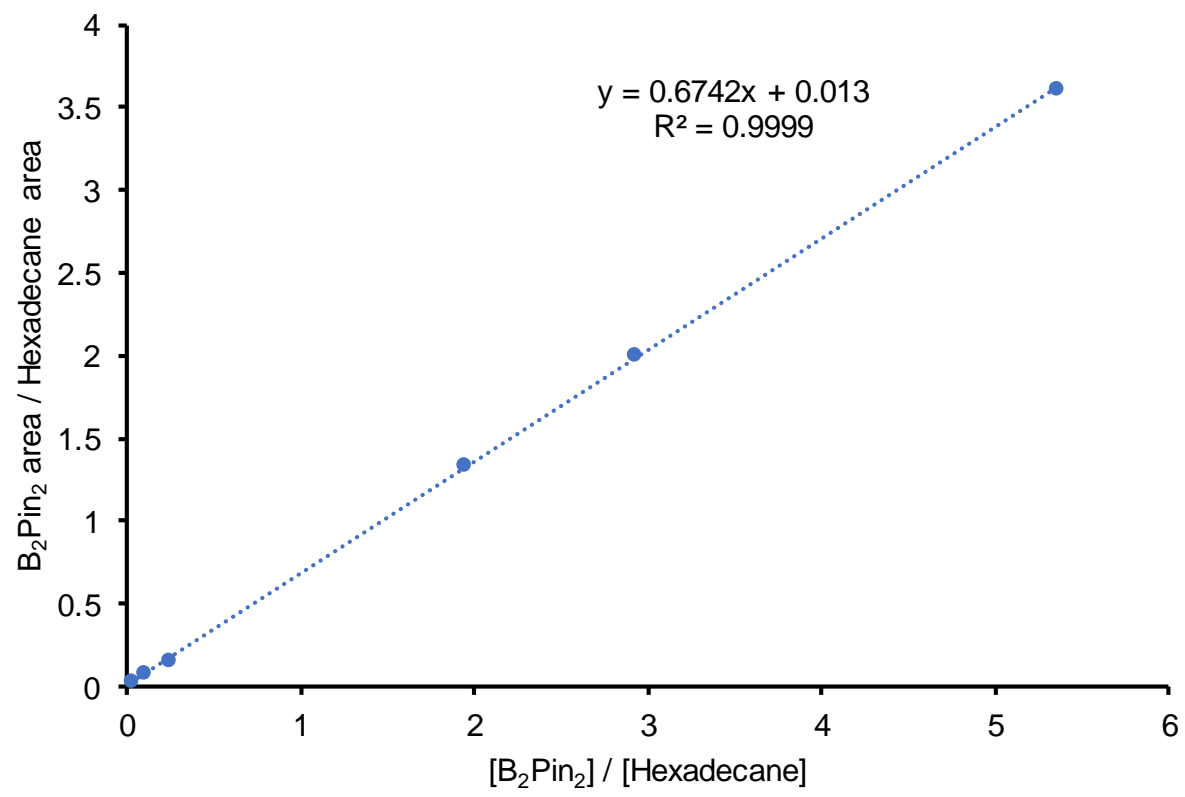

Figure S73. Calibration curve for quantification of $\left[\mathrm{B}_{2} \mathrm{Pin}_{2}\right]$ by GC-FID.

\section{PhBPin Calibration Curve}

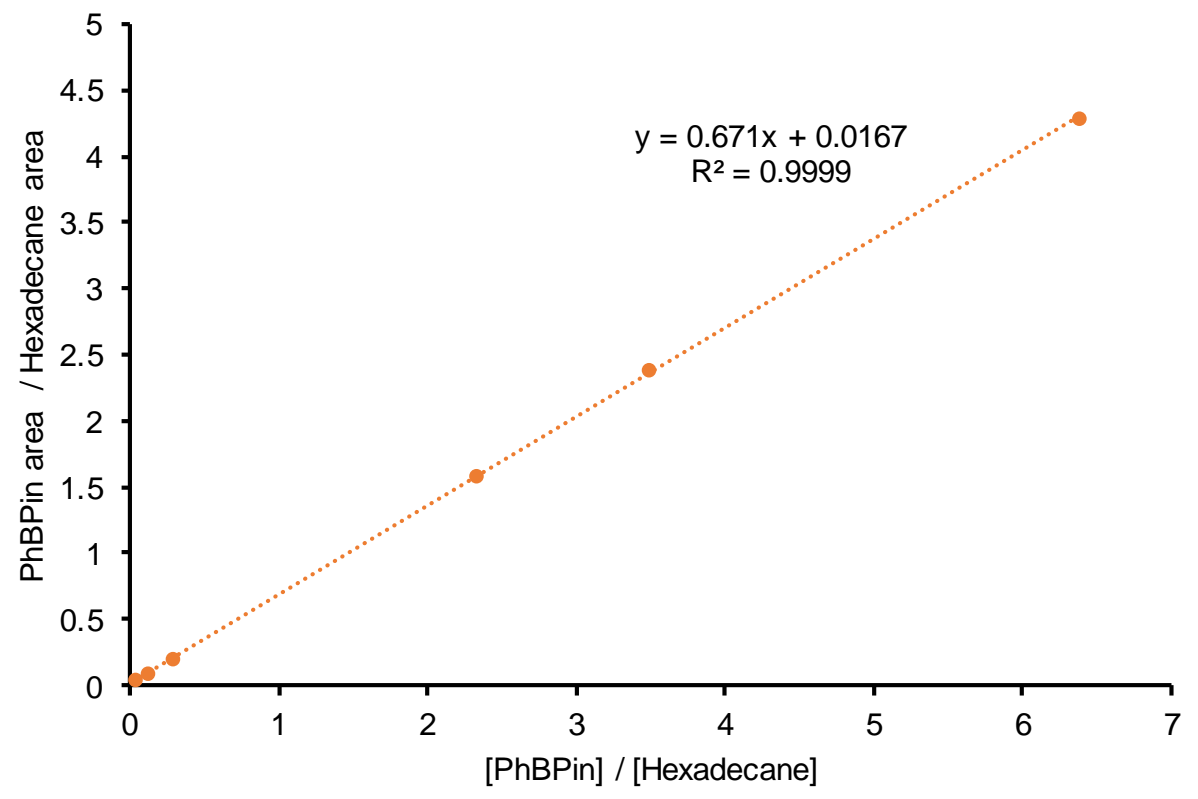

Figure S74. Calibration curve for quantification of [PhBPin] by GC-FID. 
4-methylphenylboronic acid pinacol ester Calibration Curve

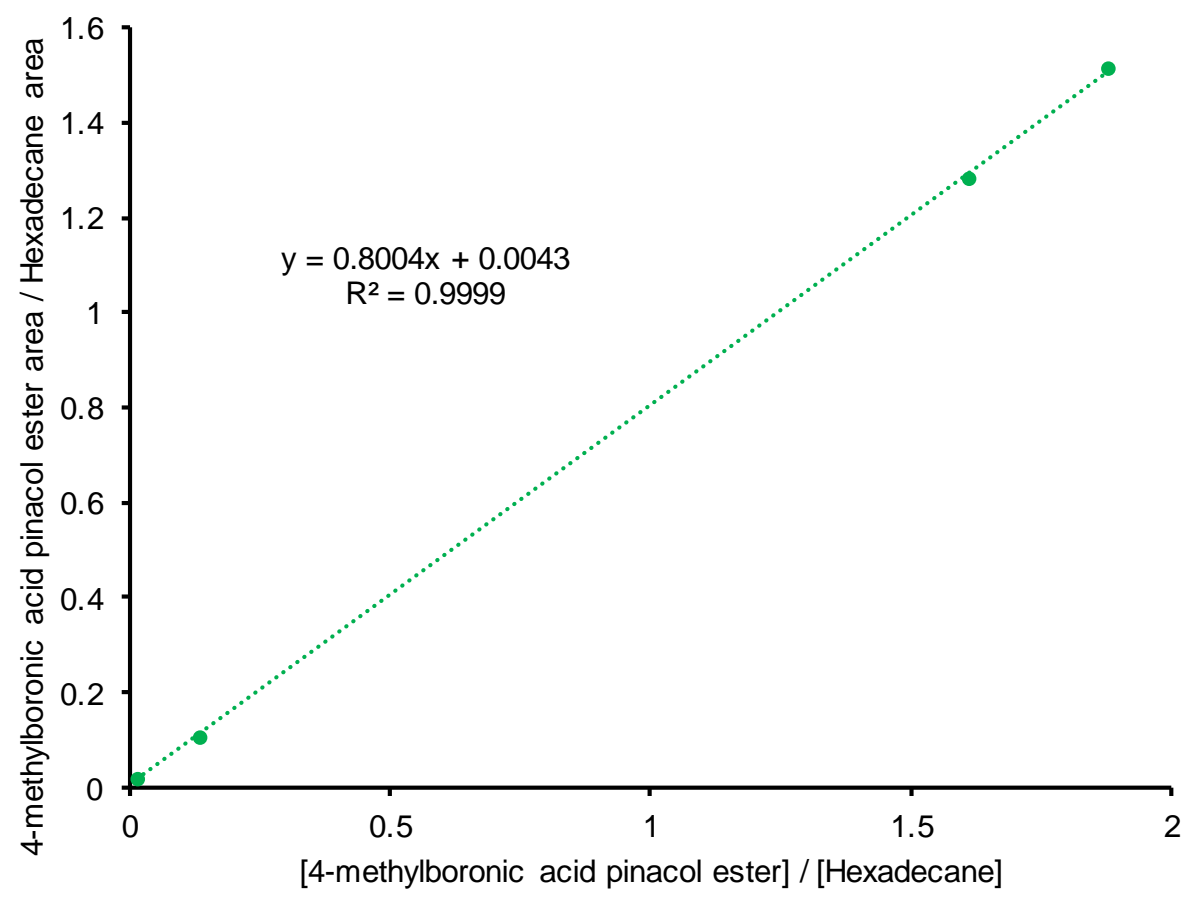

Figure S75. Calibration curve for quantification of [4-methylboronic acid pinacol ester] by GCFID.

3-methylphenylboronic acid pinacol ester Calibration Curve

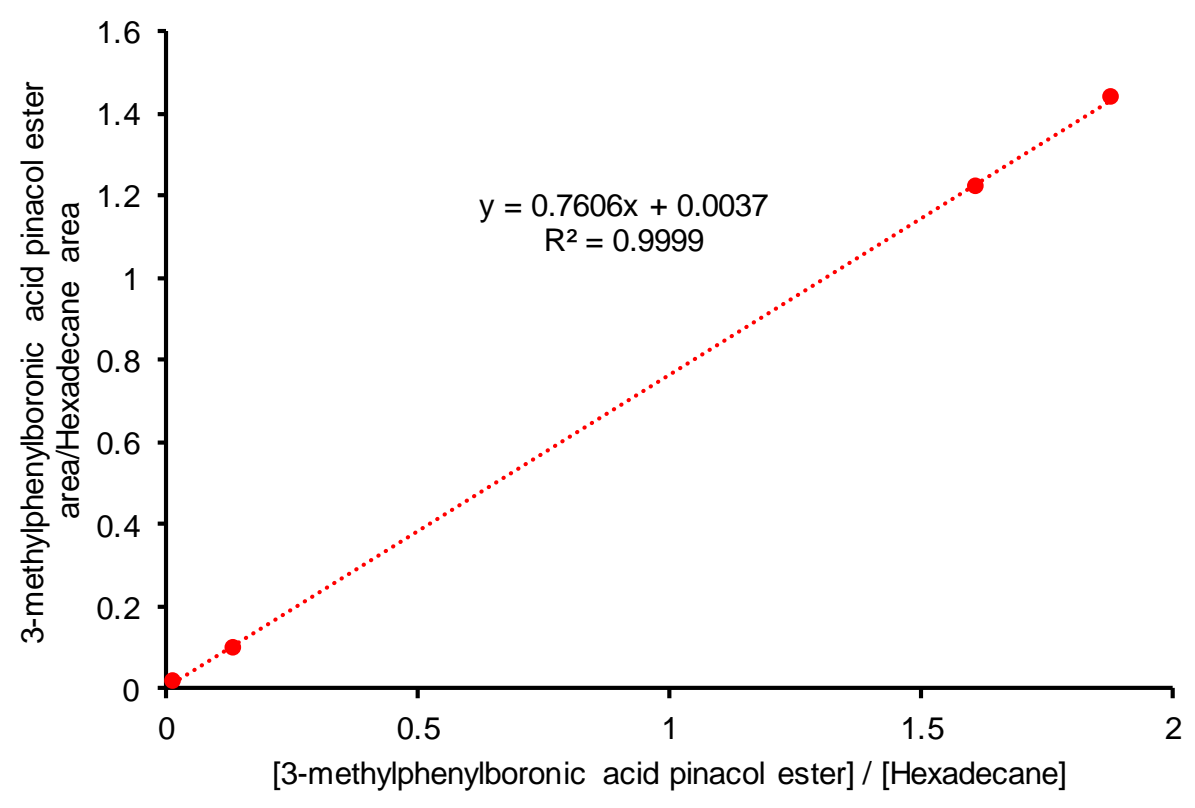


Figure S76. Calibration curve for quantification of [3-methylboronic acid pinacol ester] by GCFID.

2-methylphenylboronic acid pinacol ester Calibration

Curve

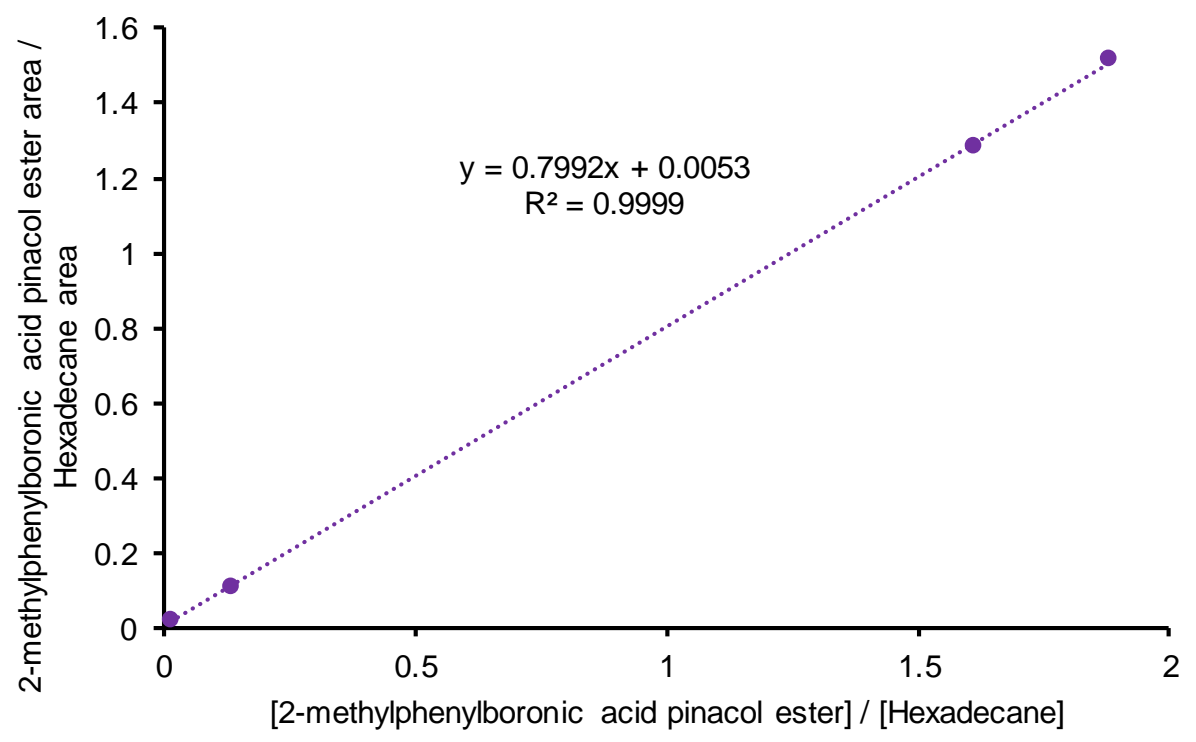

Figure S77. Calibration curve for quantification of [2-methylboronic acid pinacol ester] by GCFID.

\section{General procedure for kinetic isotope effect of heterogeneous benzene borylation reactions}

In a glovebox under inert atmosphere, to an oven-dried 2-5 $\mathrm{mL}$ Biotage microwave vial charged with stir bar was added UiO-67-mix-Ir(III) $(3,40 \mathrm{mg})$, anhydrous cyclohexane $(0.48 \mathrm{~mL})$, anhydrous benzene- $d_{6}(0.13 \mathrm{~mL}, 1.5 \mathrm{mmol})$ or anhydrous benzene $(0.13 \mathrm{~mL}, 1.5 \mathrm{mmol}), \mathrm{B}_{2} \mathrm{Pin}_{2}$ as a stock solution in anhydrous cyclohexane (56 mg, $0.22 \mathrm{mmol}$ ), and anhydrous hexadecane $(20 \mu \mathrm{L}, 0.068 \mathrm{mmol})$ as the internal standard. The microwave vial was then sealed with a cap that was crimped. It was stirred at $1000 \mathrm{rpm}$ using an aluminum block in the center of a Heidolph hotplate with magnetic stirring at room temperature and an initial aliquot was taken via needle and syringe using an outlet purge needle to relieve any pressure. The aliquot was diluted with $\sim 0.2 \mathrm{~mL}$ of anhydrous cyclohexane and solids were filtered using a GE Healthcare Whatman ${ }^{\mathrm{TM}}$ Mini-UniPrep ${ }^{\mathrm{TM}}$ Syringeless Filter vial. The microwave vial was then removed from the aluminum block and the temperature of the hotplate was set to $80^{\circ} \mathrm{C}$. The reaction was then placed on the heated aluminum block and the liquid meniscus was further wrapped in aluminum foil for insulation. The reaction was monitored over time by GC-FID.

Table S19. Conditions for Kinetic Isotope Effect Study of Benzene Borylation

\begin{tabular}{|c|c|c|c|c|c|c|c|}
\hline Entry & $\begin{array}{c}\text { Catalyst (mg, } \\
\text { targeted) }\end{array}$ & $\begin{array}{c}\text { Catalyst (mg, } \\
\text { measured) }\end{array}$ & Arene (M) & $\begin{array}{c}\mathrm{B}_{2} \mathrm{Pin}_{2} \\
(\mathrm{M}, \\
\text { targeted) }\end{array}$ & $\begin{array}{l}\mathrm{B}_{2} \mathrm{Pin}_{2}(\mathrm{M}, \\
\text { measured } \\
\text { by GC-FID) }\end{array}$ & $\begin{array}{l}\text { Hexadecane } \\
\text { (M) }\end{array}$ & $\begin{array}{c}\text { Total } \\
\text { Volume } \\
(\mathrm{mL})\end{array}$ \\
\hline
\end{tabular}




\begin{tabular}{cccccccc}
\hline 1 & 40 & 40.7 & 0.48 & 0.073 & 0.068 & 0.023 & 3.02 \\
2 & 40 & 40.6 & 0.48 & 0.073 & 0.071 & 0.023 & 3.02 \\
3 & 40 & 40.5 & 0.48 & 0.073 & 0.073 & 0.023 & 3.02 \\
$4^{a}$ & 40 & 40.8 & 0.49 & 0.073 & 0.068 & 0.023 & 3.02 \\
$5^{a}$ & 40 & 40.4 & 0.49 & 0.073 & 0.072 & 0.023 & 3.02 \\
$6^{a}$ & 40 & 40.9 & 0.49 & 0.073 & 0.070 & 0.023 & 3.02 \\
\hline
\end{tabular}

${ }^{a}$ Benzene- $d_{6}$ used

Table S20. Results for Kinetic Isotope Effect Study of Benzene Borylation (Table S19 Entries 1-3)

\begin{tabular}{|c|c|c|c|c|}
\hline Time (min) & $\begin{array}{c}\text { Average }\left[\mathrm{B}_{2} \mathrm{Pin}_{2}\right] \\
(\mathrm{M})\end{array}$ & $\begin{array}{c}\text { Average [PhBPin] } \\
\text { (M) }\end{array}$ & $\begin{array}{l}\text { STDEV.S [B } \\
\text { (M) }\end{array}$ & $\begin{array}{c}\text { STDEV.S [PhBPin] } \\
\text { (M) }\end{array}$ \\
\hline 0 & 0.0707 & 0.0000 & 0.0023 & 0.00000 \\
\hline 20 & 0.0663 & 0.0022 & 0.0024 & 0.00013 \\
\hline 40 & 0.0638 & 0.0050 & 0.0025 & 0.00041 \\
\hline 60 & 0.0593 & 0.0082 & 0.0014 & 0.00064 \\
\hline 80 & 0.0548 & 0.0121 & 0.0016 & 0.00108 \\
\hline 100 & 0.0502 & 0.0176 & 0.0019 & 0.00048 \\
\hline 120 & 0.0438 & 0.0247 & 0.0008 & 0.00167 \\
\hline 140 & 0.0350 & 0.0350 & 0.0029 & 0.00205 \\
\hline 160 & 0.0238 & 0.0538 & 0.0041 & 0.00770 \\
\hline 180 & 0.0122 & 0.0847 & 0.0043 & 0.01477 \\
\hline 200 & 0.0033 & 0.1196 & 0.0025 & 0.01243 \\
\hline 220 & 0.0000 & 0.1337 & 0.0000 & 0.00177 \\
\hline 240 & 0.0000 & 0.1346 & 0.0000 & 0.00299 \\
\hline 260 & 0.0000 & 0.1346 & 0.0000 & 0.00094 \\
\hline 280 & 0.0000 & 0.1333 & 0.0000 & 0.00264 \\
\hline
\end{tabular}


Table S21. Results for Kinetic Isotope Effect Study of Benzene Borylation (Table S19 Entries 4-6)

\begin{tabular}{|c|c|c|c|c|}
\hline Time (min) & $\begin{array}{c}\text { Average }\left[\mathrm{B}_{2} \mathrm{Pin}_{2}\right] \\
(\mathrm{M})\end{array}$ & $\begin{array}{c}\text { Average [PhBPin] } \\
\text { (M) }\end{array}$ & $\begin{array}{l}\text { STDEV.S }\left[\mathrm{B}_{2} \mathrm{Pin}_{2}\right] \\
(\mathrm{M})\end{array}$ & $\begin{array}{c}\text { STDEV.S [PhBPin] } \\
\text { (M) }\end{array}$ \\
\hline 0 & 0.0701 & 0.0000 & 0.001832 & 0.000000 \\
\hline 20 & 0.0672 & 0.0008 & 0.001529 & 0.000097 \\
\hline 40 & 0.0646 & 0.0019 & 0.002290 & 0.000055 \\
\hline 60 & 0.0632 & 0.0031 & 0.001650 & 0.000147 \\
\hline 80 & 0.0619 & 0.0041 & 0.000527 & 0.000288 \\
\hline 100 & 0.0600 & 0.0052 & 0.002498 & 0.000307 \\
\hline 120 & 0.0591 & 0.0063 & 0.001940 & 0.000300 \\
\hline 140 & 0.0579 & 0.0074 & 0.001765 & 0.000383 \\
\hline 160 & 0.0563 & 0.0086 & 0.002468 & 0.000422 \\
\hline 180 & 0.0547 & 0.0098 & 0.002569 & 0.000370 \\
\hline 200 & 0.0535 & 0.0114 & 0.002846 & 0.000298 \\
\hline 220 & 0.0514 & 0.0130 & 0.001870 & 0.000547 \\
\hline 240 & 0.0490 & 0.0148 & 0.002327 & 0.000336 \\
\hline 260 & 0.0461 & 0.0169 & 0.003087 & 0.000623 \\
\hline 280 & 0.0436 & 0.0201 & 0.002080 & 0.000518 \\
\hline
\end{tabular}




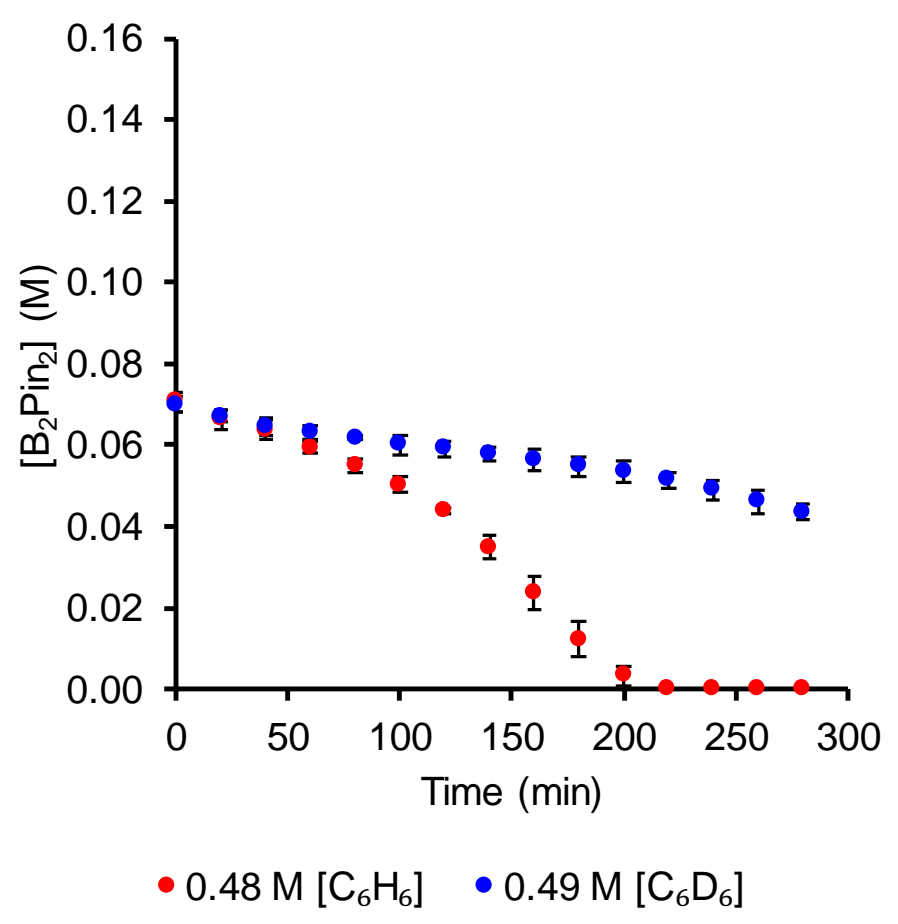

Figure S78. $\mathrm{B}_{2} \mathrm{Pin}_{2}$ consumption in benzene and benzene- $d_{6}$ borylation with error bars reflecting standard deviation (sample) of triplicate runs.

Benzene Borylation for KIE

(Triplicate)

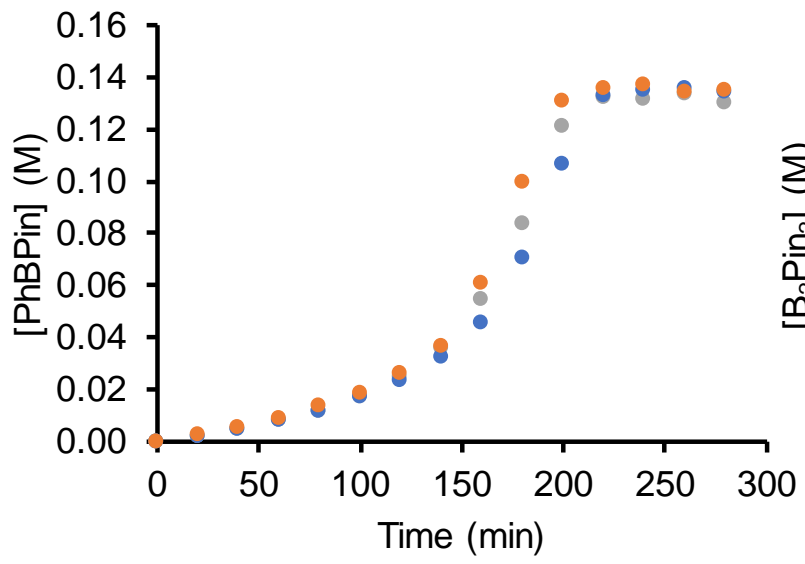

- Table S19 Entry $1 \cdot$ Table S19 Entry 2

- Table S19 Entry 3
Benzene Borylation for KIE (Triplicate)

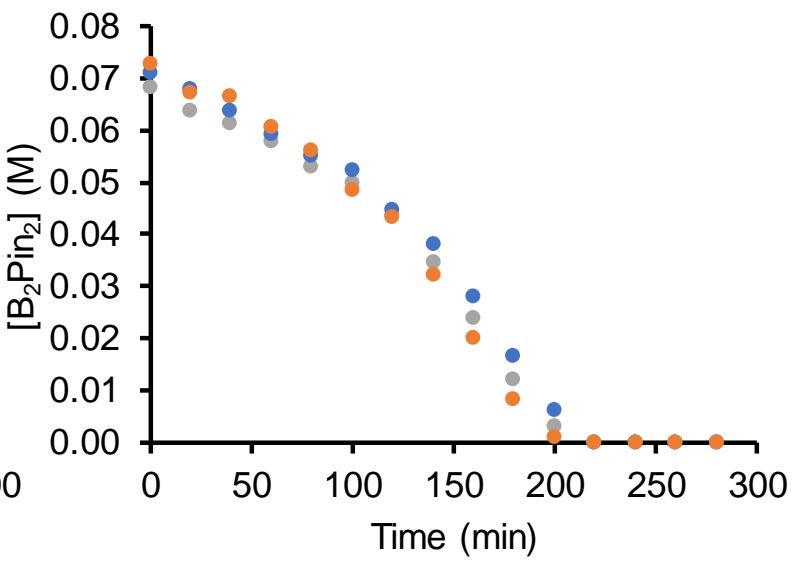

- Table S19 Entry $1 \cdot$ Table S19 Entry 2

- Table S19 Entry 3

Figure S79. $\mathrm{B}_{2} \mathrm{Pin}_{2}$ consumption and PhBPin formation in benzene, triplicate runs overlaid. 


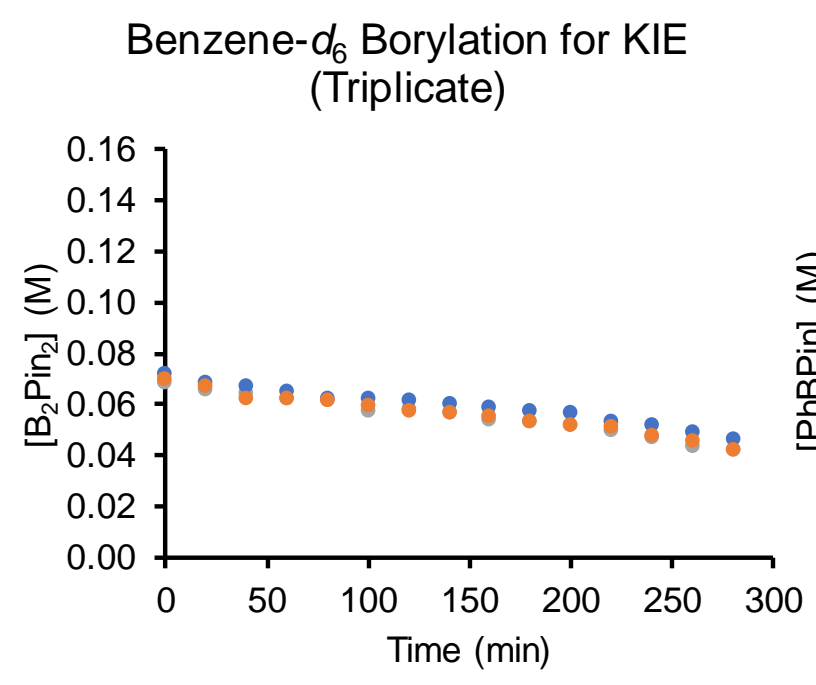

- Table S19 Entry $4 \cdot$ Table S19 Entry 5

- Table S19 Entry 6
Benzene- $d_{6}$ Borylation for KIE (Triplicate)

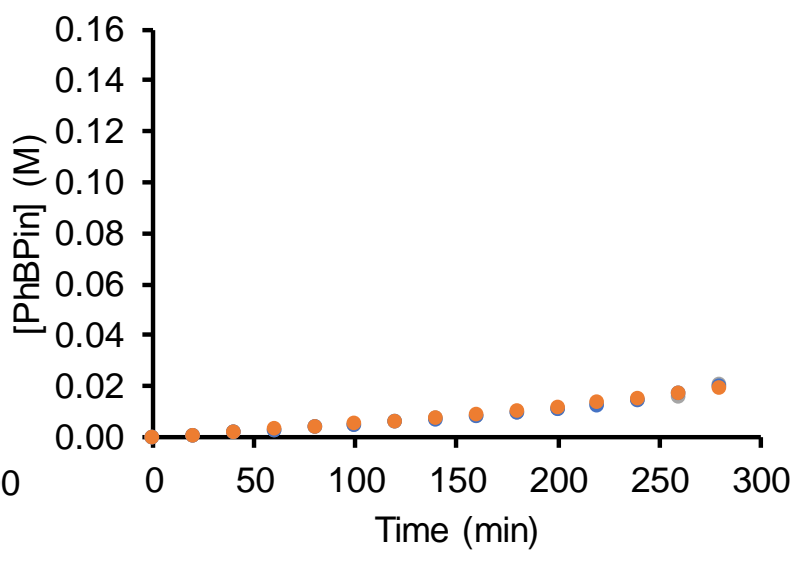

- Table S19 Entry $4 \cdot$ Table S19 Entry 5

- Table S19 Entry 6

Figure S80. $\mathrm{B}_{2} \mathrm{Pin}_{2}$ consumption and PhBPin formation in benzene- $d_{6}$, triplicate runs overlaid.

\section{General procedure for heterogeneous toluene borylation reaction}

In a glovebox under inert atmosphere, to an oven-dried 2-5 mL Biotage microwave vial charged with stir bar was added UiO-67-mix-Ir(III) (3, $13 \mathrm{mg})$, anhydrous cyclohexane $(0.45 \mathrm{~mL})$, anhydrous toluene $(0.2 \mathrm{~mL}, 1.9 \mathrm{mmol}), \mathrm{B}_{2} \mathrm{Pin}_{2}$ as a stock solution in anhydrous cyclohexane (18.2 $\mathrm{mg}, 0.072 \mathrm{mmol})$, and anhydrous hexadecane $(20 \mu \mathrm{L}, 0.068 \mathrm{mmol})$ as the internal standard. The microwave vial was then sealed with a cap that was crimped. It was stirred at $80{ }^{\circ} \mathrm{C}$ at $1000 \mathrm{rpm}$ for 280 min using an aluminum block in the center of a Heidolph hotplate with magnetic stirring. The liquid meniscus was further wrapped in aluminum foil for insulation. After 280 min, an aliquot was then taken with a needle and syringe as well as an outlet purge needle to relieve pressure. It was diluted with $\sim 0.2 \mathrm{~mL}$ of anhydrous cyclohexane and solids were filtered using a GE Healthcare Whatman ${ }^{\mathrm{TM}}$ Mini-UniPrep ${ }^{\mathrm{TM}}$ Syringeless Filter vial, after which it was analyzed by GCFID.

\section{General procedure for heterogeneous benzene borylation reaction}

In a glovebox under inert atmosphere, to an oven-dried 2-5 $\mathrm{mL}$ Biotage microwave vial charged with stir bar was added UiO-67-mix-Ir(III) (3, $13 \mathrm{mg})$, anhydrous cyclohexane $(0.48 \mathrm{~mL})$, anhydrous benzene $(0.17 \mathrm{~mL}, 1.9 \mathrm{mmol}), \mathrm{B}_{2} \mathrm{Pin}_{2}$ as a stock solution in anhydrous cyclohexane (18.2 $\mathrm{mg}, 0.072 \mathrm{mmol})$, and anhydrous hexadecane $(20 \mu \mathrm{L}, 0.068 \mathrm{mmol})$ as the internal standard. The microwave vial was then sealed with a cap that was crimped. It was stirred at $80{ }^{\circ} \mathrm{C}$ at 1000 rpm for 280 min using an aluminum block in the center of a Heidolph hotplate with magnetic stirring. The liquid meniscus was further wrapped in aluminum foil for insulation. After $280 \mathrm{~min}$, an aliquot was then taken with a needle and syringe as well as an outlet purge needle to relieve pressure. It was diluted with $\sim 0.2 \mathrm{~mL}$ of anhydrous cyclohexane and solids were filtered using a GE Healthcare Whatman ${ }^{\mathrm{TM}}$ Mini-UniPrep ${ }^{\mathrm{TM}}$ Syringeless Filter vial, after which it was analyzed by GC-FID. 


\section{General procedure for heterogeneous benzene and toluene competition borylation reaction}

In a glovebox under inert atmosphere, to an oven-dried 2-5 mL Biotage microwave vial charged with stir bar was added UiO-67-mix-Ir(III) (3, $13 \mathrm{mg})$, anhydrous cyclohexane $(0.28 \mathrm{~mL})$, anhydrous benzene $(0.17 \mathrm{~mL}, 1.9 \mathrm{mmol})$, anhydrous toluene $(0.2 \mathrm{~mL}, 1.9 \mathrm{mmol}), \mathrm{B}_{2} \mathrm{Pin}_{2}$ as a stock solution in anhydrous cyclohexane $(18.2 \mathrm{mg}, 0.072 \mathrm{mmol})$, and anhydrous hexadecane (20 $\mu \mathrm{L}, 0.068 \mathrm{mmol}$ ) as the internal standard. The microwave vial was then sealed with a cap that was crimped. It was stirred at $80{ }^{\circ} \mathrm{C}$ at $1000 \mathrm{rpm}$ for 280 min using an aluminum block in the center of a Heidolph hotplate with magnetic stirring. The liquid meniscus was further wrapped in aluminum foil for insulation. After $280 \mathrm{~min}$, an aliquot was then taken with a needle and syringe as well as an outlet purge needle to relieve pressure. It was diluted with $\sim 0.2 \mathrm{~mL}$ of anhydrous cyclohexane and solids were filtered using a GE Healthcare Whatman ${ }^{\mathrm{TM}}$ Mini-UniPrep ${ }^{\mathrm{TM}}$ Syringeless Filter vial, after which it was analyzed by GC-FID.

\section{General procedure for homogeneous benzene and toluene competition borylation reaction}

In a glovebox under inert atmosphere, to an oven-dried 2-5 mL Biotage microwave vial charged with stir bar was added $[\operatorname{Ir}(\mathrm{COD}) \mathrm{Cl}]_{2}(2.61 \mathrm{mg}, 0.0039 \mathrm{mmol})$, anhydrous cyclohexane $(0.28 \mathrm{~mL})$, anhydrous toluene $(0.2 \mathrm{~mL}, 1.9 \mathrm{mmol}), \mathrm{B}_{2} \mathrm{Pin}_{2}$ as a stock solution in anhydrous cyclohexane (18.2 $\mathrm{mg}, 0.072 \mathrm{mmol})$, anhydrous hexadecane $(20 \mu \mathrm{L}, 0.068 \mathrm{mmol})$ as the internal standard, and 1,10phenanthroline as a stock solution in benzene $(1.4 \mathrm{mg}, 0.0078 \mathrm{mmol}$, in $0.17 \mathrm{~mL}, 1.9 \mathrm{mmol}$, of benzene) The microwave vial was then sealed with a cap that was crimped. It was stirred at 80 ${ }^{\circ} \mathrm{C}$ at $1000 \mathrm{rpm}$ for 280 min using an aluminum block in the center of a Heidolph hotplate with magnetic stirring. The liquid meniscus was further wrapped in aluminum foil for insulation. After $280 \mathrm{~min}$, an aliquot was then taken with a needle and syringe as well as an outlet purge needle to relieve pressure. It was diluted with $\sim 0.2 \mathrm{~mL}$ of anhydrous cyclohexane and solids were filtered using a GE Healthcare Whatman ${ }^{\mathrm{TM}}$ Mini-UniPrep ${ }^{\mathrm{TM}}$ Syringeless Filter vial, after which it was analyzed by GC-FID.

\section{General procedure for size exclusion experiment with $t$-butylbenzene}

In a glovebox under inert atmosphere, to an oven-dried 2-5 mL Biotage microwave vial charged with stir bar was added UiO-67-mix-Ir(III) $(3,13 \mathrm{mg})$, anhydrous cyclohexane- $d_{12}(0.36 \mathrm{~mL})$, anhydrous t-butylbenzene $(0.29 \mathrm{~mL}, 1.9 \mathrm{mmol}), \mathrm{B}_{2} \mathrm{Pin}_{2}$ as a stock solution in anhydrous cyclohexane- $d_{12}(18.2 \mathrm{mg}, 0.072 \mathrm{mmol})$, and anhydrous hexadecane $(20 \mu \mathrm{L}, 0.068 \mathrm{mmol})$ as the internal standard. The microwave vial was then sealed with a cap that was crimped. It was stirred at $80{ }^{\circ} \mathrm{C}$ at $1000 \mathrm{rpm}$ for 3 hours using an aluminum block in the center of a Heidolph hotplate with magnetic stirring. The liquid meniscus was further wrapped in aluminum foil for insulation. After 3 hours, an aliquot was then taken with a needle and syringe as well as an outlet purge needle to relieve pressure. It was diluted with $\sim 0.2 \mathrm{~mL}$ of anhydrous cyclohexane and solids were filtered using a GE Healthcare Whatman ${ }^{\mathrm{TM}}$ Mini-UniPrep ${ }^{\mathrm{TM}}$ Syringeless Filter vial, after which it was analyzed by GC-FID. $0.5 \mathrm{~mL}$ were then analyzed for ${ }^{1} \mathrm{H}$ NMR to check for borylated $t$ butylbenzene.

\section{General procedure for size exclusion experiment with benzene comparison}

In a glovebox under inert atmosphere, to an oven-dried 2-5 mL Biotage microwave vial charged with stir bar was added UiO-67-mix-Ir(III) (3, $13 \mathrm{mg})$, anhydrous cyclohexane $(0.48 \mathrm{~mL})$, anhydrous benzene $(0.17 \mathrm{~mL}, 1.9 \mathrm{mmol}), \mathrm{B}_{2} \mathrm{Pin}_{2}$ as a stock solution in anhydrous cyclohexane 
(18.2 $\mathrm{mg}, 0.072 \mathrm{mmol})$, and anhydrous hexadecane $(20 \mu \mathrm{L}, 0.068 \mathrm{mmol})$ as the internal standard. The microwave vial was then sealed with a cap that was crimped. It was stirred at $80^{\circ} \mathrm{C}$ at 1000 rpm for 3 hours using an aluminum block in the center of a Heidolph hotplate with magnetic stirring. The liquid meniscus was further wrapped in aluminum foil for insulation. After 3 hours, an aliquot was then taken with a needle and syringe as well as an outlet purge needle to relieve pressure. It was diluted with $\sim 0.2 \mathrm{~mL}$ of anhydrous cyclohexane and solids were filtered using a GE Healthcare Whatman ${ }^{\mathrm{TM}}$ Mini-UniPrep ${ }^{\mathrm{TM}}$ Syringeless Filter vial, after which it was analyzed by GC-FID.

Table S22. Size Exclusion Experiment

\begin{tabular}{llll}
\hline Entry & Arene & {$\left[\mathbf{B}_{2}\right.$ Pin $\left._{2}\right](\mathbf{M})$ after 3 h } & [PhBPin] (M) after 3 h \\
\hline $1^{a}$ & t-butylbenzene & 0.073 & $\mathrm{~N} / \mathrm{A}$ \\
2 & Benzene & 0.011 & 0.12 \\
\hline
\end{tabular}

${ }^{a}$ Borylated products not observed

\section{General procedure for heterogeneous benzene borylation recycling experiment}

In a glovebox under inert atmosphere, to an oven-dried 2-5 mL Biotage microwave vial charged with stir bar was added UiO-67-mix-Ir(III) (3, $40 \mathrm{mg})$, anhydrous cyclohexane $(0.89 \mathrm{~mL})$, anhydrous benzene $(0.13 \mathrm{~mL}, 1.5 \mathrm{mmol})$, and $\mathrm{B}_{2} \mathrm{Pin}_{2}$ as a stock solution in anhydrous cyclohexane (56 mg, $0.22 \mathrm{mmol}$ ). The microwave vial was then sealed with a cap that was crimped. It was stirred at $80^{\circ} \mathrm{C}$ at $1000 \mathrm{rpm}$ for 3 hours using an aluminum block in the center of a Heidolph hotplate with magnetic stirring. The liquid meniscus was further wrapped in aluminum foil for insulation. After 3 hours, the reaction was stopped, and the material was allowed to settle. The supernatant was decanted, and fresh anhydrous cyclohexane was added and decanted. Note, some of the liquid remained in the vial. To this was then added anhydrous benzene $(0.13$ $\mathrm{mL}, 1.5 \mathrm{mmol})$, anhydrous cyclohexane $(0.87 \mathrm{~mL})$, anhydrous hexadecane $(20 \mu \mathrm{L}, 0.068 \mathrm{mmol})$, and $\mathrm{B}_{2} \mathrm{Pin}_{2}$ as a stock solution in anhydrous cyclohexane (56 mg, $\left.0.22 \mathrm{mmol}\right)$. It was stirred at $1000 \mathrm{rpm}$ using an aluminum block in the center of a Heidolph hotplate with magnetic stirring at room temperature and an initial aliquot was taken via needle and syringe using an outlet purge needle to relieve any pressure. The aliquot was diluted with $\sim 0.2 \mathrm{~mL}$ of anhydrous cyclohexane and solids were filtered using a GE Healthcare Whatman ${ }^{\mathrm{TM}}$ Mini-UniPrep ${ }^{\mathrm{TM}}$ Syringeless Filter vial. The microwave vial was then removed from the aluminum block and the temperature of the hotplate was set to $80^{\circ} \mathrm{C}$. The reaction was then placed on the heated aluminum block and the liquid meniscus was further wrapped in aluminum foil for insulation. The reaction was monitored over time by GC-FID. 


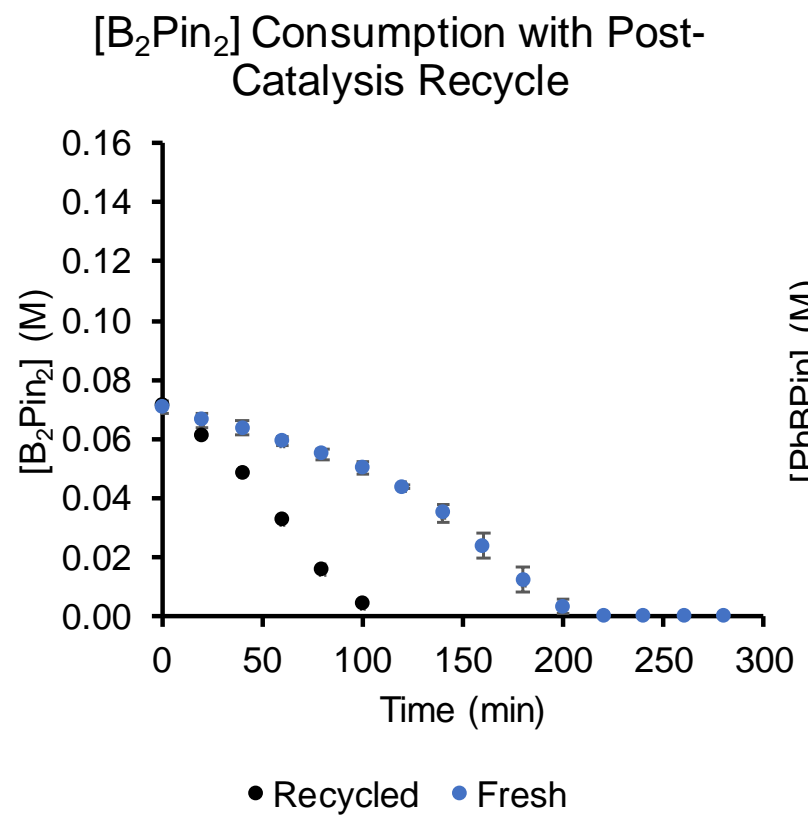

[PhBPin] Formation with Post-
Catalysis Recycle

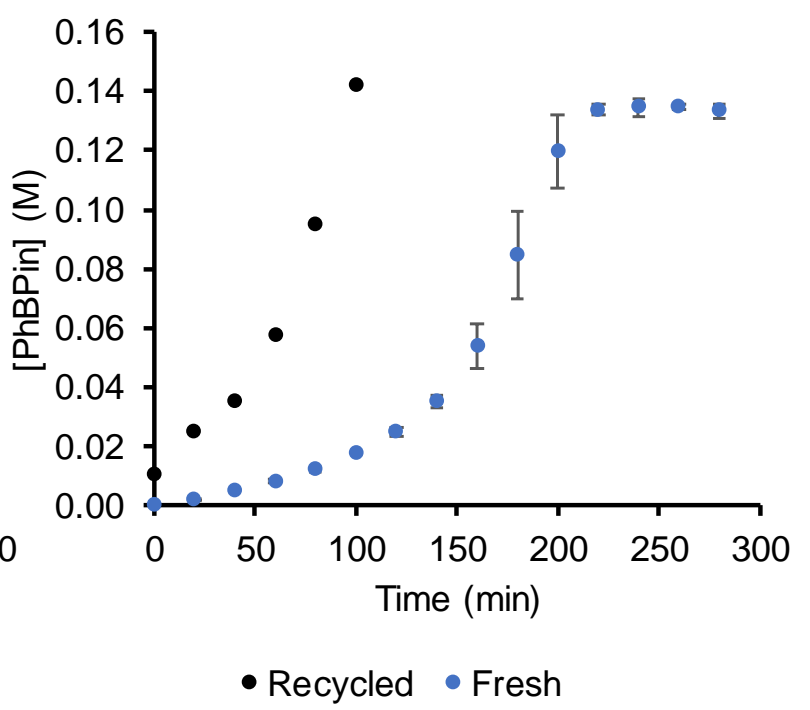

Figure S81. Comparison of kinetic profiles with fresh and recycled 3. The fresh sample plot represents data collected for Table S19 entries 1-3 with error bars representing standard deviation (sample). The reaction is observed to be faster with recycled material which may indicate a chemical transformation being responsible for the previously observed induction period, however an induction period is still present which could imply a build-up of sufficient local concentration for catalysis to proceed.

\section{References}

(1) Zhang, X.; Huang, Z.; Ferrandon, M.; Yang, D.; Robison, L.; Li, P.; Wang, T. C.; Delferro, M.; Farha, O. K. Catalytic Chemoselective Functionalization of Methane in a MetalOrganic Framework. Nat. Catal. 2018, 1 (5), 356-362. https://doi.org/10.1038/s41929018-0069-6.

(2) Seechurn, C. C. C. J.; Sivakumar, V.; Satoskar, D.; Colacot, T. J. Iridium-Catalyzed C-H Borylation of Heterocycles Using an Overlooked 1,10-Phenanthroline Ligand: Reinventing the Catalytic Activity by Understanding the Solvent-Assisted Neutral to Cationic Switch. Organometallics 2014, 33 (13), 3514-3522. https://doi.org/10.1021/om500420d.

(3) Chupas, P. J.; Qiu, X.; Hanson, J. C.; Lee, P. L.; Grey, C. P.; Billinge, S. J. L. RapidAcquisition Pair Distribution Function (RA-PDF) Analysis. J. Appl. Crystallogr. 2003, 36 (6), 1342-1347. https://doi.org/10.1107/S0021889803017564.

(4) Toby, B. H.; Von Dreele, R. B. GSAS-II: The Genesis of a Modern Open-Source All Purpose Crystallography Software Package. J. Appl. Crystallogr. 2013, 46 (2), 544-549. https://doi.org/10.1107/S0021889813003531.

(5) Qiu, X.; Thompson, J. W.; Billinge, S. J. L. PDFgetX2: A GUI-Driven Program to Obtain the Pair Distribution Function from X-Ray Powder Diffraction Data. J. Appl. Crystallogr. 2004, 37 (4), 678. https://doi.org/10.1107/S0021889804011744.

(6) Yang, X.; Juhas, P.; Farrow, C. L.; Billinge, S. J. L. XPDFsuite: An End-to-End Software 
Solution for High Throughput Pair Distribution Function Transformation, Visualization and Analysis. arXiv:1402.3163 [cond-mat] 2014.

(7) Wojdyr, M. Fityk: A General-Purpose Peak Fitting Program. J. Appl. Crystallogr. 2010, 43 (5 PART 1), 1126-1128. https://doi.org/10.1107/S0021889810030499.

(8) Perl, J.; Shin, J.; Schümann, J.; Faddegon, B.; Paganetti, H. TOPAS: An Innovative Proton Monte Carlo Platform for Research and Clinical Applications. Med. Phys. 2012, 39 (11), 6818-6837. https://doi.org/10.1118/1.4758060.

(9) Pettersen, E. F.; Goddard, T. D.; Huang, C. C.; Couch, G. S.; Greenblatt, D. M.; Meng, E. C.; Ferrin, T. E. UCSF Chimera - A Visualization System for Exploratory Research and Analysis. J. Comput. Chem. 2004, 25 (13), 1605-1612. https://doi.org/10.1002/jcc.20084.

(10) Ravel, B.; Newville, M. ATHENA, ARTEMIS, HEPHAESTUS: Data Analysis for X-Ray Absorption Spectroscopy Using IFEFFIT. J. Synchrotron Radiat. 2005, 12 (4), 537-541. https://doi.org/10.1107/S0909049505012719. 